Supporting information

\title{
From 3-Acyl-2-methylindoles to $\gamma$-Carbolines: Li-Promoted Cycloaddition Reaction and Its Quantum Chemical Study
}

Julia V. Vyalyh, Konstantin F. Suzdalev, Anton V. Lisovin, Mikhail E. Kletskii, Oleg N. Burov, and Sergey V. Kurbatov 


\section{Table of contents}

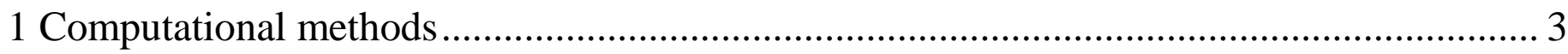

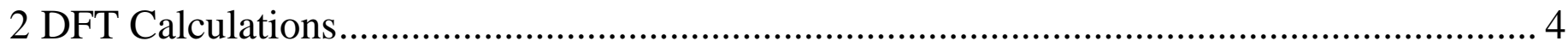

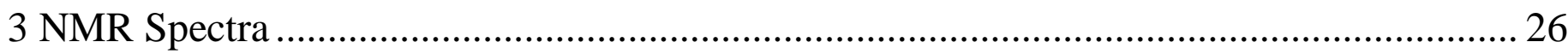

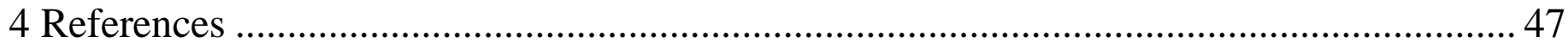




\section{Computational methods}

DFT calculations were performed using B3LYP exchange-correlation functionals ${ }^{1}$ together with the standard $6-311++\mathrm{G}(\mathrm{d}, \mathrm{p})$ basis set. For some systems ab initio calculations in the same basis were performed, and second-order Møller-Plesset perturbation (MP2) theory was taken into account. Geometry optimization was carried out using Berny's analytical gradient optimization method. Stationary points of the minimum energy reaction paths (MEPs) were characterized by frequency calculations. MEPs were obtained via the gradient descent method from the transition states in both direct and reverse directions of the transition vectors. When choosing the $6-31++G(d, p)$ basis for the theoretical study of such systems by the B3LYP functional, we were guided by literature data for cyclic systems: G-bases of types 6-31 and 6-311 give a small general error and good results (geometric characteristics). ${ }^{2}$ The solvent effects have been considered in the context of a polarizable continuum model $(\mathrm{PCM}) .^{3}$ All computations were carried out using the Gaussian 09 suite of programs ${ }^{4}$ using the Silver cluster of the Research Institute of Physical and Organic Chemistry at Southern Federal University. We also performed a topological analysis of electron density distribution function according to the "atoms in molecules" (AIM) model. ${ }^{5}$ 


\section{DFT Calculations}

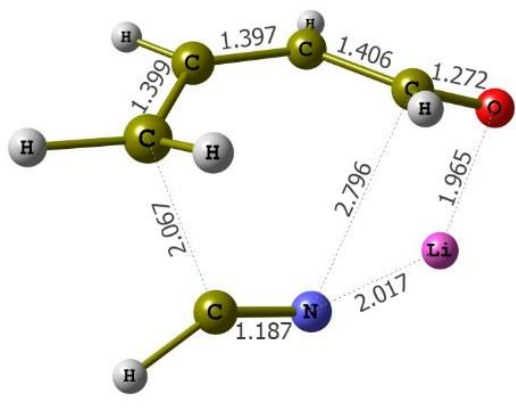

exo-1

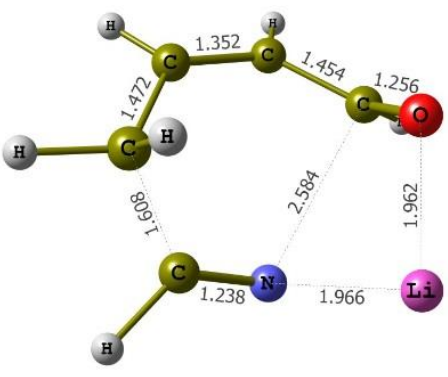

endo-1

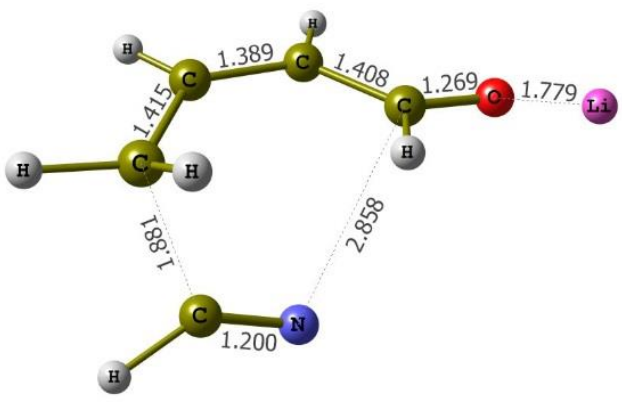

exo-2

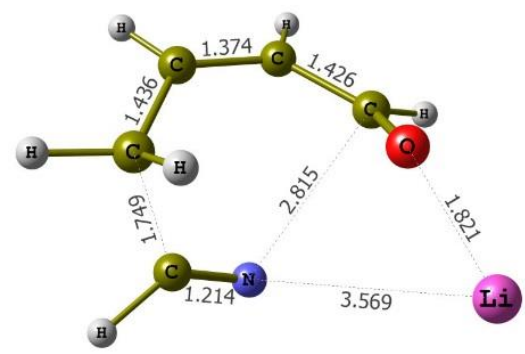

endo-2

Fig. S1. Transition states structures for the pathways for interaction of lithium enolate $\mathbf{1 9}$ and HCN molecule. The main interatomic distances are in angstroms. PCM/B3LYP/6-311++G(d,p) calculations in THF.

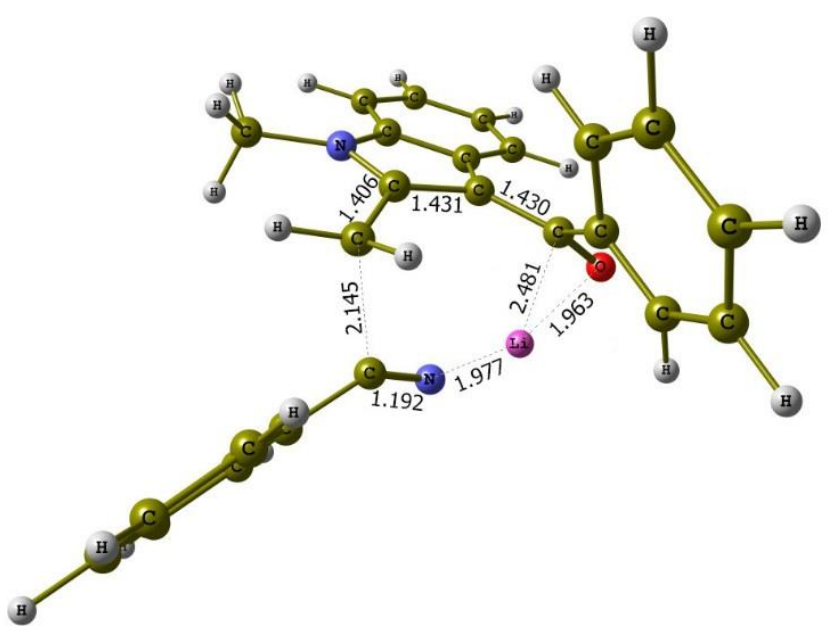

TS1

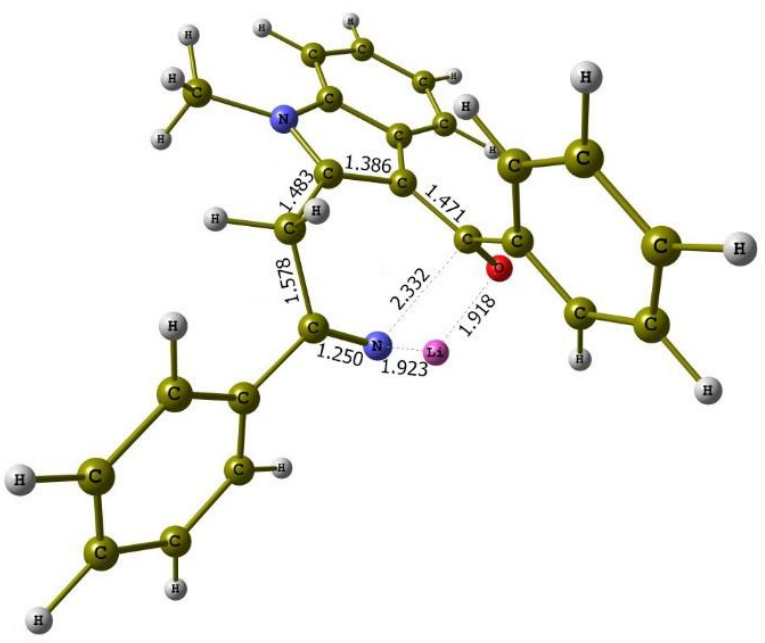

TS2

Fig. S2. Transition states structures for the first (TS1') and second (TS2') steps of PhCN cycloaddition to lithium enolate 15. The main interatomic distances are in angstroms. PCM/B3LYP/6-311++G(d,p) calculations in THF. 
Table S1. Optimized geometries of stationary points of four ways of interaction of lithium butadienolate 19 with hydrogen cyanide molecule. PCM/B3LYP/6-311++G(d,p) calculations $\left(\mathrm{THF},-78^{\circ} \mathrm{C}\right)$. Coordinates are in angstroms. Sum of electronic and thermal Free Energies in a.u. are in parentheses.

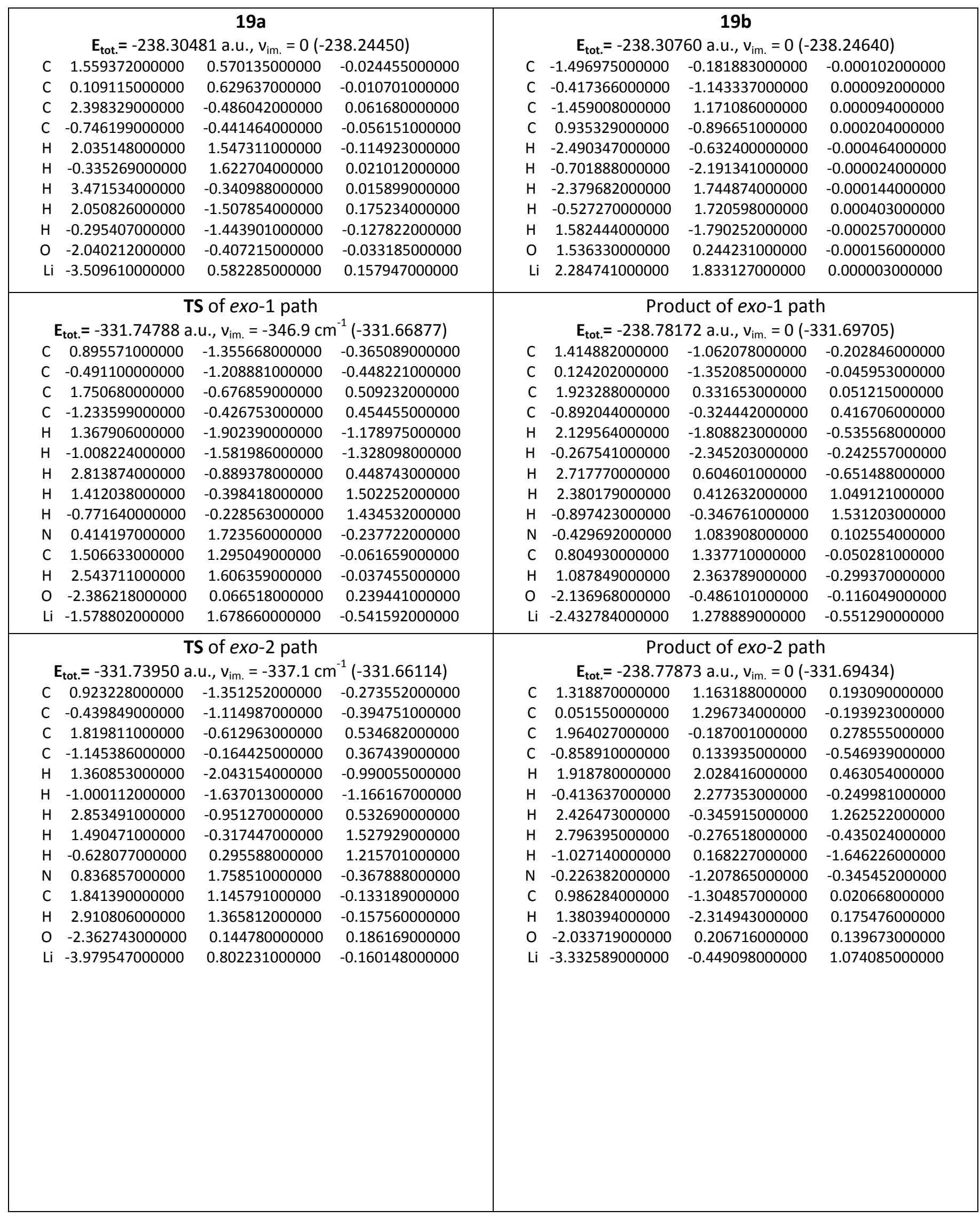




\begin{tabular}{|c|c|c|c|c|c|c|}
\hline \multicolumn{3}{|c|}{$\begin{array}{c}\text { TS1 of endo-1 path } \\
E_{\text {tot. }}=-331.75150 \text { a.u., } v_{\text {im. }}=-329.2 \mathrm{~cm}^{-1}(-331.67263)\end{array}$} & \multicolumn{4}{|c|}{ 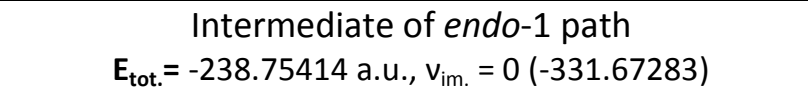 } \\
\hline C -0.255463000000 & -1.502291000000 & -0.051487000000 & c & & & -0.065018000000 \\
\hline C -1.432752000000 & -0.876034000000 & -0.476017000000 & C & -1.398917000000 & -0.937925000000 & -0.428327000000 \\
\hline C $\quad 0.750492000000$ & -1.025974000000 & 0.808590000000 & C & 0.863283000000 & -0.883094000000 & 0.747462000000 \\
\hline C -1.896433000000 & 0.411144000000 & -0.142437000000 & C & -1.926758000000 & 0.348224000000 & -0.078429000000 \\
\hline H $\quad-0.090717000000$ & -2.493691000000 & -0.471937000000 & $\mathrm{H}$ & 0.012653000000 & -2.472984000000 & -0.454274000000 \\
\hline H $\quad-2.104687000000$ & -1.455794000000 & -1.101114000000 & $\mathrm{H}$ & -2.081751000000 & -1.562571000000 & -0.996406000000 \\
\hline H $\quad 1.394723000000$ & -1.783821000000 & 1.247428000000 & $\mathrm{H}$ & 1.414784000000 & -1.653419000000 & 1.291724000000 \\
\hline H $\quad 0.505600000000$ & -0.207310000000 & 1.474944000000 & $\mathrm{H}$ & 0.499805000000 & -0.121070000000 & 1.433429000000 \\
\hline H $\quad-2.934823000000$ & 0.626052000000 & -0.447899000000 & $\mathrm{H}$ & -3.014057000000 & 0.458830000000 & -0.229572000000 \\
\hline$N \quad 2.006057000000$ & 1.094244000000 & -0.435087000000 & $\mathrm{~N}$ & 1.967638000000 & 1.081517000000 & -0.439931000000 \\
\hline C 2.079422000000 & -0.088120000000 & -0.278376000000 & C & 1.978326000000 & -0.126510000000 & -0.202707000000 \\
\hline H $\quad 2.750566000000$ & -0.901163000000 & -0.548538000000 & $\mathrm{H}$ & 2.717070000000 & -0.874231000000 & -0.551326000000 \\
\hline $0 \quad-1.268753000000$ & 1.334908000000 & 0.449697000000 & 0 & -1.301046000000 & 1.333121000000 & 0.358670000000 \\
\hline Li $\quad 0.371789000000$ & 2.121470000000 & 0.044504000000 & $\mathrm{Li}$ & 0.379693000000 & 2.149623000000 & -0.040434000000 \\
\hline \multicolumn{3}{|c|}{$\begin{array}{c}\text { TS2 of endo-1 path } \\
E_{\text {tot. }}=-331.73965 \text { a.u., } v_{\text {im. }}=-159.1 \mathrm{~cm}^{-1}(-331.65873)\end{array}$} & \multicolumn{4}{|c|}{$\begin{array}{l}\text { Product of endo-1 path } \\
38.78172 \text { a.u., } v_{\text {im. }}=0(-331.69709)\end{array}$} \\
\hline C $\quad 0.823228000000$ & -1.428415000000 & -0.019373000000 & C & -1.414776000000 & -1.062095000000 & -0.203006000000 \\
\hline C -0.442721000000 & -1.403765000000 & -0.492435000000 & C & -0.124253000000 & -1.352181000000 & -0.044890000000 \\
\hline C 1.463331000000 & -0.291858000000 & 0.662370000000 & c & -1.923224000000 & 0.331833000000 & 0.049548000000 \\
\hline C -1.418098000000 & -0.354949000000 & -0.244040000000 & C & 0.892103000000 & -0.324591000000 & 0.417381000000 \\
\hline H $\quad 1.465422000000$ & -2.245540000000 & -0.336106000000 & $\mathrm{H}$ & -2.129322000000 & -1.809104000000 & -0.535425000000 \\
\hline H $\quad-0.754313000000$ & -2.157012000000 & -1.213014000000 & $\mathrm{H}$ & 0.267398000000 & -2.345582000000 & -0.240332000000 \\
\hline H $\quad 2.520673000000$ & -0.477708000000 & 0.859026000000 & $\mathrm{H}$ & -2.382666000000 & 0.413131000000 & 1.046265000000 \\
\hline H $\quad 0.984418000000$ & -0.043120000000 & 1.613966000000 & $\mathrm{H}$ & -2.715976000000 & 0.604876000000 & -0.655134000000 \\
\hline H $\quad-2.107056000000$ & -0.182844000000 & -1.091104000000 & $\mathrm{H}$ & 0.898731000000 & -0.347315000000 & 1.531839000000 \\
\hline$N \quad 0.331698000000$ & 1.460843000000 & -0.806808000000 & $\mathrm{~N}$ & 0.429898000000 & 1.083890000000 & 0.104287000000 \\
\hline C 1.322924000000 & 1.073912000000 & -0.173648000000 & C & -0.804611000000 & 1.337747000000 & -0.049676000000 \\
\hline H $\quad 2.251779000000$ & 1.666696000000 & -0.042638000000 & $\mathrm{H}$ & -1.087212000000 & 2.364094000000 & -0.298056000000 \\
\hline $0 \quad-1.676156000000$ & 0.202071000000 & 0.851701000000 & 0 & 2.136473000000 & -0.485842000000 & -0.116903000000 \\
\hline Li $\quad-1.255181000000$ & 2.009169000000 & 0.215556000000 & $\mathrm{Li}$ & 2.432180000000 & 1.278375000000 & -0.553361000000 \\
\hline \multicolumn{3}{|c|}{$\begin{array}{c}\text { TS of endo-2 path } \\
E_{\text {tot. }}=-331.73087 \text { a.u., } v_{\text {im. }}=-184.4 \mathrm{~cm}^{-1}(-331.65192)\end{array}$} & \multicolumn{4}{|c|}{$\begin{array}{l}\text { Product of endo-2 path } \\
238.77868 \text { a.u., } v_{\text {im. }}=0(-331.69494)\end{array}$} \\
\hline C 0.912250000000 & 1.336147000000 & -0.237838000000 & C & 1.372767000000 & 1.122585000000 & -0.196860000000 \\
\hline C -0.257332000000 & 1.444398000000 & 0.475901000000 & C & 0.097112000000 & 1.311741000000 & 0.135837000000 \\
\hline C 1.300320000000 & 0.140316000000 & -0.931486000000 & C & 1.973121000000 & -0.251198000000 & -0.210705000000 \\
\hline C -1.353865000000 & 0.535465000000 & 0.415001000000 & C & -0.860441000000 & 0.193021000000 & 0.505605000000 \\
\hline H $\quad 1.687087000000$ & 2.071601000000 & -0.034331000000 & $\mathrm{H}$ & 2.011373000000 & 1.956819000000 & -0.475005000000 \\
\hline H $\quad-0.339746000000$ & 2.217563000000 & 1.235312000000 & $\mathrm{H}$ & -0.338196000000 & 2.307309000000 & 0.138387000000 \\
\hline H $\quad 2.2787480000000$ & 0.188488000000 & -1.408958000000 & $\mathrm{H}$ & 2.741960000000 & -0.358083000000 & 0.568974000000 \\
\hline H $\quad 0.555344000000$ & -0.275547000000 & -1.605677000000 & $\mathrm{H}$ & 2.505014000000 & -0.444712000000 & -1.151994000000 \\
\hline H $\quad-2.035637000000$ & 0.578360000000 & 1.281447000000 & $\mathrm{H}$ & -1.010196000000 & 0.239626000000 & 1.607795000000 \\
\hline$N \quad 0.605637000000$ & -1.345397000000 & 1.154514000000 & $\mathrm{~N}$ & -0.286055000000 & -1.175673000000 & 0.303810000000 \\
\hline C 1.362952000000 & -1.174365000000 & 0.220814000000 & c & 0.934984000000 & -1.326340000000 & -0.012948000000 \\
\hline H $\quad 2.185759000000$ & -1.810196000000 & -0.142960000000 & $\mathrm{H}$ & 1.287206000000 & -2.351525000000 & -0.168493000000 \\
\hline $0 \quad-1.688951000000$ & -0.197119000000 & -0.555669000000 & 0 & -2.041874000000 & 0.311261000000 & -0.165690000000 \\
\hline Li -2.281786000000 & -1.889104000000 & -0.871811000000 & $\mathrm{Li}$ & -3.321679000000 & -0.636221000000 & -0.882127000000 \\
\hline \multicolumn{3}{|c|}{$E_{\text {tot. }}=-93.46046$ a.u., $v_{\text {im. }}=0(-93.45578)$} & & & & \\
\hline 00000000000 & 0.000000000000 & 0.650896 & & & & \\
\hline C 0.000000000000 & 0.000000000000 & -0.49813 & & & & \\
\hline H $\quad 0.000000000000$ & 0.000000000000 & -1.567483000000 & & & & \\
\hline
\end{tabular}


Table S2. Optimized geometries of stationary points of four ways of interaction of lithium butadienolate 19 with hydrogen cyanide molecule. PCM/MP2//HF/6-311++G(d,p) calculations (THF, $\left.-78^{\circ} \mathrm{C}\right)$. Coordinates are in angstroms. Sum of electronic and thermal Free Energies in a.u. are in parentheses.

\begin{tabular}{|c|c|c|c|c|c|c|c|}
\hline \multicolumn{4}{|c|}{ 19a } & \multicolumn{4}{|c|}{$19 \mathrm{~b}$} \\
\hline \multicolumn{4}{|c|}{$E_{\text {tot. }}=-237.56801$ a.u., $v_{\text {im. }}=0(-237.50206)$} & \multicolumn{4}{|c|}{$E_{\text {tot. }}=-238.30760$ a.u., $v_{\text {im. }}=0(-238.24640)$} \\
\hline $\mathrm{C}$ & 1.557460000000 & 0.561279000000 & -0.055745000000 & $\mathrm{C}$ & -1.491733000000 & -0.185928000000 & 0.000045000000 \\
\hline $\mathrm{C}$ & 0.098300000000 & 0.630920000000 & 0.021287000000 & $\mathrm{C}$ & -0.392968000000 & -1.143081000000 & -0.000031000000 \\
\hline $\mathrm{C}$ & 2.373696000000 & -0.483635000000 & 0.094357000000 & $\mathrm{C}$ & -1.475640000000 & 1.150882000000 & -0.000040000000 \\
\hline C & -0.742957000000 & -0.426637000000 & -0.094555000000 & $\mathrm{C}$ & 0.940605000000 & -0.877451000000 & -0.000005000000 \\
\hline $\mathrm{H}$ & 2.031969000000 & 1.511486000000 & -0.259601000000 & $\mathrm{H}$ & -2.469556000000 & -0.647977000000 & 0.000189000000 \\
\hline $\mathrm{H}$ & -0.330593000000 & 1.613823000000 & 0.145707000000 & $\mathrm{H}$ & -0.668283000000 & -2.184065000000 & -0.000078000000 \\
\hline $\mathrm{H}$ & 3.436831000000 & -0.367698000000 & -0.027343000000 & $\mathrm{H}$ & -2.401553000000 & 1.700993000000 & 0.000046000000 \\
\hline $\mathrm{H}$ & 2.018499000000 & -1.471539000000 & 0.335477000000 & $\mathrm{H}$ & -0.564420000000 & 1.717344000000 & -0.000197000000 \\
\hline $\mathrm{H}$ & -0.288957000000 & -1.400123000000 & -0.279107000000 & $\mathrm{H}$ & 1.588025000000 & -1.756239000000 & -0.000046000000 \\
\hline $\mathrm{O}$ & -2.018297000000 & -0.412947000000 & -0.027891000000 & $\mathrm{O}$ & 1.526439000000 & 0.250499000000 & 0.000023000000 \\
\hline $\mathrm{Li}$ & -3.480124000000 & 0.575352000000 & 0.171974000000 & $\mathrm{Li}$ & 2.274231000000 & 1.833141000000 & 0.000032000000 \\
\hline \multicolumn{4}{|c|}{ Product of exo-1 path } & \multicolumn{4}{|c|}{ Product of exo-2 path } \\
\hline \multicolumn{4}{|c|}{$E_{\text {tot. }}=-330.80041$ a.u., $v_{\text {im. }}=0(-330.70717)$} & \multicolumn{4}{|c|}{$E_{\text {tot. }}=-330.79576$ a.u., $v_{\text {im. }}=0(-330.70309)$} \\
\hline $\mathrm{C}$ & 1.408793000000 & -1.055172000000 & -0.194858000000 & $\mathrm{C}$ & 1.319507000000 & 1.154735000000 & 0.189277000000 \\
\hline $\mathrm{C}$ & 0.137418000000 & -1.347728000000 & -0.000148000000 & $\mathrm{C}$ & 0.059348000000 & 1.289694000000 & -0.175457000000 \\
\hline $\mathrm{C}$ & 1.911159000000 & 0.350715000000 & -0.027108000000 & $\mathrm{C}$ & 1.958536000000 & -0.198181000000 & 0.269228000000 \\
\hline C & -0.892113000000 & -0.324531000000 & 0.429818000000 & $\mathrm{C}$ & -0.854653000000 & 0.134200000000 & -0.534596000000 \\
\hline $\mathrm{H}$ & 2.119274000000 & -1.809794000000 & -0.488995000000 & $\mathrm{H}$ & 1.921516000000 & 2.011902000000 & 0.444350000000 \\
\hline $\mathrm{H}$ & -0.231332000000 & -2.351912000000 & -0.128093000000 & $\mathrm{H}$ & -0.397213000000 & 2.265114000000 & -0.226985000000 \\
\hline $\mathrm{H}$ & 2.588889000000 & 0.623166000000 & -0.832375000000 & $\mathrm{H}$ & 2.411733000000 & -0.360802000000 & 1.245964000000 \\
\hline $\mathrm{H}$ & 2.486508000000 & 0.459143000000 & 0.892274000000 & $\mathrm{H}$ & 2.773395000000 & -0.291303000000 & -0.448290000000 \\
\hline $\mathrm{H}$ & -0.971787000000 & -0.388951000000 & 1.524889000000 & $\mathrm{H}$ & -1.009873000000 & 0.177969000000 & -1.621961000000 \\
\hline $\mathrm{N}$ & -0.432467000000 & 1.068035000000 & 0.181671000000 & $\mathrm{~N}$ & -0.230662000000 & -1.188560000000 & -0.326355000000 \\
\hline $\mathrm{C}$ & 0.774813000000 & 1.337211000000 & -0.006847000000 & $\mathrm{C}$ & 0.967126000000 & -1.302170000000 & 0.014403000000 \\
\hline $\mathrm{H}$ & 1.038712000000 & 2.371796000000 & -0.190521000000 & $\mathrm{H}$ & 1.352983000000 & -2.307036000000 & 0.150349000000 \\
\hline 0 & -2.089616000000 & -0.484094000000 & -0.171462000000 & 0 & -2.023841000000 & 0.216858000000 & 0.126454000000 \\
\hline $\mathrm{Li}$ & -2.442160000000 & 1.243363000000 & -0.627442000000 & $\mathrm{Li}$ & -3.315454000000 & -0.460155000000 & 1.050767000000 \\
\hline \multicolumn{4}{|c|}{ Product of endo-1 path } & \multicolumn{4}{|c|}{ Product of endo-2 path } \\
\hline \multicolumn{4}{|c|}{$E_{\text {tot. }}=-330.80041$ a.u., $v_{\text {im. }}=0(-330.70717)$} & \multicolumn{4}{|c|}{$E_{\text {tot. }}=-330.79606$ a.u., $v_{\text {im. }}=0(-330.70347)$} \\
\hline $\mathrm{C}$ & -1.408806000000 & -1.055170000000 & -0.194846000000 & $\mathrm{C}$ & 1.371303000000 & 1.114879000000 & -0.188026000000 \\
\hline C & -0.137427000000 & -1.347727000000 & -0.000154000000 & $\mathrm{C}$ & 0.106398000000 & 1.304790000000 & 0.133299000000 \\
\hline $\mathrm{C}$ & -1.911165000000 & 0.350724000000 & -0.027100000000 & $\mathrm{C}$ & 1.960558000000 & -0.262759000000 & -0.219107000000 \\
\hline $\mathrm{C}$ & 0.892114000000 & -0.324531000000 & 0.429803000000 & $\mathrm{C}$ & -0.857555000000 & 0.192535000000 & 0.495719000000 \\
\hline $\mathrm{H}$ & -2.119295000000 & -1.809793000000 & -0.488960000000 & $\mathrm{H}$ & 2.012983000000 & 1.942767000000 & -0.442862000000 \\
\hline $\mathrm{H}$ & 0.231317000000 & -2.351915000000 & -0.128088000000 & $\mathrm{H}$ & -0.315683000000 & 2.296610000000 & 0.148813000000 \\
\hline $\mathrm{H}$ & -2.486509000000 & 0.459160000000 & 0.892282000000 & $\mathrm{H}$ & 2.733855000000 & -0.377680000000 & 0.540209000000 \\
\hline $\mathrm{H}$ & -2.588894000000 & 0.623175000000 & -0.832368000000 & $\mathrm{H}$ & 2.455416000000 & -0.456077000000 & -1.169475000000 \\
\hline $\mathrm{H}$ & 0.971781000000 & -0.388945000000 & 1.524874000000 & $\mathrm{H}$ & -1.014319000000 & 0.251096000000 & 1.582693000000 \\
\hline $\mathrm{N}$ & 0.432467000000 & 1.068029000000 & 0.181645000000 & $\mathrm{~N}$ & -0.287604000000 & -1.156909000000 & 0.300487000000 \\
\hline $\mathrm{C}$ & -0.774811000000 & 1.337215000000 & -0.006857000000 & $\mathrm{C}$ & 0.914360000000 & -1.323443000000 & -0.001855000000 \\
\hline $\mathrm{H}$ & -1.038714000000 & 2.371801000000 & -0.190521000000 & $\mathrm{H}$ & 1.259433000000 & -2.343994000000 & -0.129833000000 \\
\hline 0 & 2.089621000000 & -0.484105000000 & -0.171465000000 & 0 & -2.020661000000 & 0.316897000000 & -0.171809000000 \\
\hline $\mathrm{Li}$ & 2.442213000000 & 1.243363000000 & -0.627366000000 & $\mathrm{Li}$ & -3.307849000000 & -0.635182000000 & -0.859554000000 \\
\hline \multicolumn{4}{|c|}{$\mathrm{HCN}$} & & & & \\
\hline \multicolumn{4}{|c|}{$E_{\text {tot. }}=-93.20479$ a.u., $v_{\text {im. }}=0(-93.19874)$} & & & & \\
\hline $\mathrm{N}$ & 0.000000000000 & 0.000000000000 & 0.639578000000 & & & & \\
\hline C & 0.000000000000 & 0.000000000000 & -0.488037000000 & & & & \\
\hline $\mathrm{H}$ & 0.000000000000 & 0.000000000000 & -1.548822000000 & & & & \\
\hline
\end{tabular}


Table S3. Optimized geometries of stationary points of two ways of interaction of lithium butadienolate 19a with benzonitrile molecule. PCM/B3LYP/6-311++G(d,p) calculations $\left(\mathrm{THF},-78^{\circ} \mathrm{C}\right)$. Coordinates are in angstroms. Sum of electronic and thermal Free Energies in a.u. are in parentheses.

\begin{tabular}{|c|c|c|c|c|c|c|c|}
\hline \multicolumn{4}{|c|}{$\begin{array}{c}\text { TS of exo-1 path } \\
E_{\text {tot. }}=-562.86269 \text { a.u., } v_{\text {im. }}=-341.0 \mathrm{~cm}^{-1}(-562.70539)\end{array}$} & \multicolumn{4}{|c|}{$\begin{array}{c}\text { Product of exo-1 path } \\
E_{\text {tot. }}=-562.89680 \text { a.u., } v_{\text {im. }}=0(-562.73393)\end{array}$} \\
\hline C & -1.920588000000 & 1.391657000000 & 1.040929000000 & $\mathrm{C}$ & -2.297844000000 & -1.735156000000 & -0.565704000000 \\
\hline C & -3.073171000000 & 0.648148000000 & 0.820193000000 & $\mathrm{C}$ & -3.098062000000 & -0.695128000000 & -0.347101000000 \\
\hline C & -0.866906000000 & 1.571810000000 & 0.126729000000 & $\mathrm{C}$ & -0.886998000000 & -1.739624000000 & -0.039648000000 \\
\hline C & -3.377380000000 & 0.071150000000 & -0.432558000000 & C & -2.635503000000 & 0.506982000000 & 0.450950000000 \\
\hline $\mathrm{H}$ & -1.733758000000 & 1.702720000000 & 2.066765000000 & $\mathrm{H}$ & -2.624719000000 & -2.601156000000 & -1.133084000000 \\
\hline $\mathrm{H}$ & -3.696681000000 & 0.366520000000 & 1.664345000000 & $\mathrm{H}$ & -4.115541000000 & -0.657622000000 & -0.722269000000 \\
\hline $\mathrm{H}$ & -0.043704000000 & 2.201382000000 & 0.446495000000 & $\mathrm{H}$ & -0.227306000000 & -2.296482000000 & -0.709369000000 \\
\hline $\mathrm{H}$ & -1.090847000000 & 1.654595000000 & -0.933243000000 & $\mathrm{H}$ & -0.839893000000 & -2.261897000000 & 0.927369000000 \\
\hline $\mathrm{H}$ & -2.899797000000 & 0.525048000000 & -1.314454000000 & $\mathrm{H}$ & -2.801488000000 & 0.267190000000 & 1.527601000000 \\
\hline N & -0.960603000000 & -1.183968000000 & -0.320043000000 & $\mathrm{~N}$ & -1.135528000000 & 0.672922000000 & 0.361363000000 \\
\hline C & -0.153399000000 & -0.316910000000 & -0.150061000000 & $\mathrm{C}$ & -0.366105000000 & -0.322008000000 & 0.142418000000 \\
\hline 0 & -4.162795000000 & -0.904750000000 & -0.626046000000 & 0 & -3.216702000000 & 1.685622000000 & 0.084398000000 \\
\hline C & 1.316292000000 & -0.189544000000 & -0.098119000000 & C & 1.108953000000 & -0.100626000000 & 0.045685000000 \\
\hline C & 2.000859000000 & 0.770571000000 & -0.853066000000 & $\mathrm{C}$ & 2.013021000000 & -1.123407000000 & 0.367133000000 \\
\hline C & 2.044875000000 & -1.073208000000 & 0.710428000000 & $\mathrm{C}$ & 1.623166000000 & 1.141989000000 & -0.353424000000 \\
\hline C & 3.391420000000 & 0.836187000000 & -0.810094000000 & $\mathrm{C}$ & 3.388905000000 & -0.905002000000 & 0.307663000000 \\
\hline C & 3.434463000000 & -0.988416000000 & 0.767640000000 & C & 2.995523000000 & 1.356884000000 & -0.426554000000 \\
\hline C & 4.112192000000 & -0.036879000000 & 0.005525000000 & $\mathrm{C}$ & 3.885377000000 & 0.334237000000 & -0.091761000000 \\
\hline $\mathrm{H}$ & 1.448044000000 & 1.458582000000 & -1.481046000000 & $\mathrm{H}$ & 1.648146000000 & -2.093599000000 & 0.682291000000 \\
\hline $\mathrm{H}$ & 1.517706000000 & -1.818536000000 & 1.293989000000 & $\mathrm{H}$ & 0.936652000000 & 1.935459000000 & -0.620086000000 \\
\hline $\mathrm{H}$ & 3.911818000000 & 1.574264000000 & -1.409940000000 & $\mathrm{H}$ & 4.071164000000 & -1.705222000000 & 0.571363000000 \\
\hline $\mathrm{H}$ & 3.987449000000 & -1.669520000000 & 1.404764000000 & $\mathrm{H}$ & 3.373202000000 & 2.320810000000 & -0.749094000000 \\
\hline $\mathrm{H}$ & 5.193630000000 & 0.024939000000 & 0.047044000000 & $\mathrm{H}$ & 4.955011000000 & 0.501945000000 & -0.146641000000 \\
\hline $\mathrm{Li}$ & -2.673076000000 & -2.200537000000 & -0.433773000000 & $\mathrm{Li}$ & -1.691839000000 & 2.693433000000 & 0.076416000000 \\
\hline \multicolumn{4}{|c|}{ TS1 of endo-1 path } & \multicolumn{4}{|c|}{ Intermediate of endo-1 path } \\
\hline C & -1.729804000000 & -1.422301000000 & 0.164362000000 & $\mathrm{C}$ & -1.660940000000 & -1.380623000000 & 0.009860000000 \\
\hline C & -2.919201000000 & -1.309747000000 & -0.558214000000 & $\mathrm{C}$ & -2.863144000000 & -1.294568000000 & -0.642593000000 \\
\hline C & -1.161279000000 & -0.543184000000 & 1.112090000000 & $\mathrm{C}$ & -1.101408000000 & -0.484829000000 & 1.017976000000 \\
\hline C & -3.884416000000 & -0.280505000000 & -0.510830000000 & $\mathrm{C}$ & -3.894609000000 & -0.308796000000 & -0.483657000000 \\
\hline $\mathrm{H}$ & -1.158672000000 & -2.322906000000 & -0.057150000000 & $\mathrm{H}$ & -1.042609000000 & -2.230205000000 & -0.272929000000 \\
\hline $\mathrm{H}$ & -3.182485000000 & -2.142931000000 & -1.202515000000 & $\mathrm{H}$ & -3.125894000000 & -2.103345000000 & -1.318104000000 \\
\hline $\mathrm{H}$ & -0.447301000000 & -0.992818000000 & 1.796662000000 & $\mathrm{H}$ & -0.497530000000 & -1.042468000000 & 1.736463000000 \\
\hline $\mathrm{H}$ & -1.828706000000 & 0.163004000000 & 1.588645000000 & $\mathrm{H}$ & -1.865219000000 & 0.077763000000 & 1.546545000000 \\
\hline $\mathrm{H}$ & -4.827570000000 & -0.511879000000 & -1.035470000000 & $\mathrm{H}$ & -4.878337000000 & -0.609216000000 & -0.883309000000 \\
\hline $\mathrm{N}$ & -0.619268000000 & 1.826167000000 & -0.062914000000 & $\mathrm{~N}$ & -0.612610000000 & 1.796516000000 & 0.089010000000 \\
\hline C & -0.063843000000 & 0.792852000000 & 0.203131000000 & $\mathrm{C}$ & -0.137543000000 & 0.684348000000 & 0.348901000000 \\
\hline $\mathrm{O}$ & -3.801773000000 & 0.846948000000 & 0.046522000000 & 0 & -3.803914000000 & 0.819200000000 & 0.034915000000 \\
\hline C & 1.335036000000 & 0.303096000000 & 0.075397000000 & C & 1.301762000000 & 0.262950000000 & 0.147202000000 \\
\hline C & 1.700727000000 & -1.045756000000 & 0.102173000000 & C & 1.758337000000 & -1.054949000000 & 0.277061000000 \\
\hline C & 2.337738000000 & 1.270562000000 & -0.109559000000 & $\mathrm{C}$ & 2.235198000000 & 1.250960000000 & -0.206070000000 \\
\hline C & 3.033782000000 & -1.423043000000 & -0.058033000000 & $\mathrm{C}$ & 3.099089000000 & -1.377383000000 & 0.055751000000 \\
\hline C & 3.668035000000 & 0.893771000000 & -0.257456000000 & C & 3.572132000000 & 0.935460000000 & -0.419483000000 \\
\hline C & 4.021993000000 & -0.456754000000 & -0.233921000000 & $\mathrm{C}$ & 4.012193000000 & -0.384739000000 & -0.291223000000 \\
\hline $\mathrm{H}$ & 0.950340000000 & -1.812068000000 & 0.236980000000 & $\mathrm{H}$ & 1.076501000000 & -1.850788000000 & 0.548593000000 \\
\hline $\mathrm{H}$ & 2.055096000000 & 2.315752000000 & -0.134620000000 & $\mathrm{H}$ & 1.879483000000 & 2.269200000000 & -0.307822000000 \\
\hline $\mathrm{H}$ & 3.297653000000 & -2.474446000000 & -0.042377000000 & $\mathrm{H}$ & 3.426941000000 & -2.406198000000 & 0.157833000000 \\
\hline $\mathrm{H}$ & 4.429582000000 & 1.653631000000 & -0.393390000000 & $\mathrm{H}$ & 4.274583000000 & 1.717638000000 & -0.687377000000 \\
\hline $\mathrm{H}$ & 5.058629000000 & -0.751556000000 & -0.351223000000 & $\mathrm{H}$ & 5.054071000000 & -0.633842000000 & -0.459197000000 \\
\hline $\mathrm{Li}$ & -2.543369000000 & 2.214502000000 & 0.029279000000 & $\mathrm{Li}$ & -2.502933000000 & 2.198425000000 & 0.051521000000 \\
\hline
\end{tabular}




\begin{tabular}{|c|c|c|c|c|c|c|c|}
\hline \multicolumn{4}{|c|}{$\begin{array}{c}\text { TS2 of endo-1 path } \\
E_{\text {tot. }}=-562.85337 \text { a.u., } v_{\text {im. }}=-121.4 \mathrm{~cm}^{-1}(-562.69459)\end{array}$} & \multicolumn{4}{|c|}{$\begin{array}{c}\text { Product of endo-1 path } \\
\mathrm{E}_{\text {tot. }}=-562.89680 \text { a.u., } v_{\text {im. }}=0(-562.73393)\end{array}$} \\
\hline C & 2.154919000000 & 1.343247000000 & -0.963791000000 & $\mathrm{C}$ & -2.297844000000 & -1.735156000000 & -0.565704000000 \\
\hline C & 3.187354000000 & 0.472613000000 & -0.947159000000 & $\mathrm{C}$ & -3.098062000000 & -0.695128000000 & -0.347101000000 \\
\hline C & 1.012992000000 & 1.289690000000 & -0.036660000000 & $\mathrm{C}$ & -0.886998000000 & -1.739624000000 & -0.039648000000 \\
\hline C & 3.418773000000 & -0.545267000000 & 0.064247000000 & $\mathrm{C}$ & -2.635503000000 & 0.506982000000 & 0.450950000000 \\
\hline $\mathrm{H}$ & 2.067034000000 & 2.006681000000 & -1.819791000000 & $\mathrm{H}$ & -2.624719000000 & -2.601156000000 & -1.133084000000 \\
\hline $\mathrm{H}$ & 3.850191000000 & 0.419774000000 & -1.808148000000 & $\mathrm{H}$ & -4.115541000000 & -0.657622000000 & -0.722269000000 \\
\hline $\mathrm{H}$ & 0.296191000000 & 2.077983000000 & -0.260069000000 & $\mathrm{H}$ & -0.227306000000 & -2.296482000000 & -0.709369000000 \\
\hline $\mathrm{H}$ & 1.322272000000 & 1.390672000000 & 1.006755000000 & $\mathrm{H}$ & -0.839893000000 & -2.261897000000 & 0.927369000000 \\
\hline $\mathrm{H}$ & 3.876039000000 & -1.468698000000 & -0.337712000000 & $\mathrm{H}$ & -2.801488000000 & 0.267190000000 & 1.527601000000 \\
\hline $\mathrm{N}$ & 0.902488000000 & -1.241250000000 & -0.089389000000 & $\mathrm{~N}$ & -1.135528000000 & 0.672922000000 & 0.361363000000 \\
\hline C & 0.284246000000 & -0.162302000000 & -0.079385000000 & $\mathrm{C}$ & -0.366105000000 & -0.322008000000 & 0.142418000000 \\
\hline 0 & 3.333323000000 & -0.422207000000 & 1.308728000000 & 0 & -3.216702000000 & 1.685622000000 & 0.084398000000 \\
\hline C & -1.224634000000 & -0.063780000000 & -0.035734000000 & C & 1.108953000000 & -0.100626000000 & 0.045685000000 \\
\hline C & -1.894412000000 & 0.918900000000 & 0.705651000000 & C & 2.013021000000 & -1.123407000000 & 0.367133000000 \\
\hline C & -1.988330000000 & -1.016558000000 & -0.724830000000 & C & 1.623166000000 & 1.141989000000 & -0.353424000000 \\
\hline C & -3.288464000000 & 0.940691000000 & 0.766597000000 & C & 3.388905000000 & -0.905002000000 & 0.307663000000 \\
\hline C & -3.379669000000 & -0.980955000000 & -0.685501000000 & C & 2.995523000000 & 1.356884000000 & -0.426554000000 \\
\hline C & -4.036621000000 & -0.003808000000 & 0.065519000000 & C & 3.885377000000 & 0.334237000000 & -0.091761000000 \\
\hline $\mathrm{H}$ & -1.331992000000 & 1.664252000000 & 1.256183000000 & $\mathrm{H}$ & 1.648146000000 & -2.093599000000 & 0.682291000000 \\
\hline $\mathrm{H}$ & -1.472014000000 & -1.781833000000 & -1.292432000000 & $\mathrm{H}$ & 0.936652000000 & 1.935459000000 & -0.620086000000 \\
\hline $\mathrm{H}$ & -3.788423000000 & 1.698977000000 & 1.359488000000 & $\mathrm{H}$ & 4.071164000000 & -1.705222000000 & 0.571363000000 \\
\hline $\mathrm{H}$ & -3.953429000000 & -1.716290000000 & -1.239395000000 & $\mathrm{H}$ & 3.373202000000 & 2.320810000000 & -0.749094000000 \\
\hline $\mathrm{H}$ & -5.120000000000 & 0.021149000000 & 0.102173000000 & $\mathrm{H}$ & 4.955011000000 & 0.501945000000 & -0.146641000000 \\
\hline $\mathrm{Li}$ & 1.931066000000 & -1.800358000000 & 1.471708000000 & $\mathrm{Li}$ & -1.691839000000 & 2.693433000000 & 0.076416000000 \\
\hline \multicolumn{3}{|r|}{ PhCN } & $E_{\text {tot. }}=-324.58464$ a.u., $v_{\text {im. }}=0(-324.50386)$ & & & & \\
\hline $\mathrm{N}$ & 3.195752000000 & 0.000003000000 & -0.000051000000 & & & & \\
\hline $\mathrm{C}$ & 2.039386000000 & -0.000008000000 & -0.000025000000 & & & & \\
\hline C & 0.608657000000 & -0.000021000000 & -0.000007000000 & & & & \\
\hline C & -0.089162000000 & -1.217685000000 & 0.000002000000 & & & & \\
\hline$C$ & -0.089147000000 & 1.217679000000 & 0.000003000000 & & & & \\
\hline $\mathrm{C}$ & -1.479696000000 & -1.210122000000 & 0.000021000000 & & & & \\
\hline $\mathrm{C}$ & -1.479661000000 & 1.210142000000 & 0.000021000000 & & & & \\
\hline $\mathrm{C}$ & -2.174630000000 & 0.000008000000 & 0.000030000000 & & & & \\
\hline $\mathrm{H}$ & 0.457414000000 & -2.152432000000 & -0.000005000000 & & & & \\
\hline $\mathrm{H}$ & 0.457476000000 & 2.152398000000 & -0.000004000000 & & & & \\
\hline $\mathrm{H}$ & -2.020648000000 & -2.148750000000 & 0.000028000000 & & & & \\
\hline $\mathrm{H}$ & -2.020622000000 & 2.148766000000 & 0.000028000000 & & & & \\
\hline $\mathrm{H}$ & -3.258368000000 & 0.000035000000 & 0.000044000000 & & & & \\
\hline
\end{tabular}


Table S4. Optimized geometries of stationary points of two ways of interaction of lithium butadienolate 19a with benzonitrile molecule. PCM/MP2//HF/6-311++G(d,p) calculations. Coordinates are in angstroms. Sum of electronic and thermal Free Energies in a.u. are in parentheses.

\begin{tabular}{|c|c|c|c|c|c|c|c|}
\hline \multicolumn{4}{|c|}{$\begin{array}{c}\text { Product } \\
E_{\text {tot. }}=-561.22154 \text { a.u., } v_{\text {im. }}=0(-561.04506)\end{array}$} & \multicolumn{4}{|c|}{$\begin{array}{c}\text { PhCN } \\
E_{\text {tot. }}=-323.62314 \text { a.u., } v_{\text {im. }}=0(-323.53559)\end{array}$} \\
\hline c & -2.332054000000 & -1.713152000000 & -0.558205000000 & $\mathrm{~N}$ & 3.172958000000 & 0.000179000000 & -0.000182000000 \\
\hline c & -3.105767000000 & -0.680279000000 & -0.293177000000 & C & 2.040901000000 & -0.000336000000 & 0.000057000000 \\
\hline c & -0.889994000000 & -1.726266000000 & -0.132090000000 & C & 0.597756000000 & -0.000154000000 & 0.000033000000 \\
\hline c & -2.602905000000 & 0.520844000000 & 0.474044000000 & C & -0.088953000000 & -1.209103000000 & 0.000026000000 \\
\hline $\mathrm{H}$ & -2.700789000000 & -2.576249000000 & -1.086611000000 & C & -0.088666000000 & 1.208960000000 & 0.000026000000 \\
\hline $\mathrm{H}$ & -4.139969000000 & -0.654283000000 & -0.592193000000 & C & -1.472115000000 & -1.203762000000 & 0.000019000000 \\
\hline H & -0.274765000000 & -2.201130000000 & -0.888537000000 & C & -1.471829000000 & 1.203951000000 & 0.000019000000 \\
\hline $\mathrm{H}$ & -0.766991000000 & -2.318719000000 & 0.773960000000 & C & -2.161109000000 & 0.000176000000 & 0.000016000000 \\
\hline $\mathrm{H}$ & -2.785233000000 & 0.319025000000 & 1.540335000000 & $\mathrm{H}$ & 0.453332000000 & -2.136343000000 & 0.000030000000 \\
\hline $\mathrm{N}$ & -1.124459000000 & 0.652185000000 & 0.378078000000 & $\mathrm{H}$ & 0.453842000000 & 2.136070000000 & 0.000030000000 \\
\hline c & -0.376377000000 & -0.321397000000 & 0.121086000000 & $\mathrm{H}$ & -2.009076000000 & -2.134359000000 & 0.000017000000 \\
\hline 0 & -3.154927000000 & 1.688363000000 & 0.083801000000 & $\mathrm{H}$ & -2.008568000000 & 2.134676000000 & 0.000017000000 \\
\hline C & 1.106402000000 & -0.103693000000 & 0.040633000000 & $\mathrm{H}$ & -3.236141000000 & 0.000305000000 & 0.000009000000 \\
\hline C & 1.995039000000 & -1.109634000000 & 0.405077000000 & & & & \\
\hline C & 1.622184000000 & 1.115027000000 & -0.393031000000 & & & & \\
\hline C & 3.365299000000 & -0.897763000000 & 0.356513000000 & & & & \\
\hline C & 2.988420000000 & 1.323558000000 & -0.456105000000 & & & & \\
\hline C & 3.866209000000 & 0.317881000000 & -0.076300000000 & & & & \\
\hline $\mathrm{H}$ & 1.628936000000 & -2.061992000000 & 0.741887000000 & & & & \\
\hline H & 0.951156000000 & 1.896715000000 & -0.693641000000 & & & & \\
\hline $\mathrm{H}$ & 4.036093000000 & -1.683729000000 & 0.654069000000 & & & & \\
\hline H & 3.368619000000 & 2.267577000000 & -0.803898000000 & & & & \\
\hline H & 4.928159000000 & 0.480650000000 & -0.122448000000 & & & & \\
\hline $\mathrm{Li}$ & -1.651104000000 & 2.703063000000 & 0.076489000000 & & & & \\
\hline
\end{tabular}


Table S5. Optimized geometries of stationary points of interaction of lithium 1-phenylbuta-1,3-dien-1-olate $\mathbf{2 0}$ and HCN. PCM/B3LYP/6-311++G(d,p) calculations $\left(\mathrm{THF},-78^{\circ} \mathrm{C}\right)$. Coordinates are in angstroms. Sum of electronic and thermal Free Energies in a.u. are in parentheses.

\begin{tabular}{|c|c|c|c|c|c|c|c|}
\hline \multicolumn{4}{|c|}{$\begin{array}{c}20 \\
E_{\text {tot. }}=-469.41405 \text { a.u., } v_{\text {im. }}=0(-469.27627)\end{array}$} & \multicolumn{4}{|c|}{$\begin{array}{c}\text { TS of exo-1 path } \\
E_{\text {tot. }}=-562.85811 \text { a.u., } v_{\text {im. }}=-340.6 \mathrm{~cm}^{-1}(-562.70159)\end{array}$} \\
\hline C & -2.332241000000 & -1.523778000000 & 0.000353000000 & $\mathrm{C}$ & 1.889789000000 & -1.355939000000 & -0.823206000000 \\
\hline C & -2.305664000000 & -0.072750000000 & -0.000034000000 & $\mathrm{C}$ & 1.540557000000 & -0.130273000000 & -1.385882000000 \\
\hline C & -1.359183000000 & -2.462751000000 & 0.000717000000 & C & 1.515635000000 & -1.858723000000 & 0.429404000000 \\
\hline $\mathrm{C}$ & -1.247637000000 & 0.805647000000 & -0.000094000000 & C & 0.540595000000 & 0.794539000000 & -0.948991000000 \\
\hline $\mathrm{H}$ & -3.350241000000 & -1.915373000000 & 0.000336000000 & $\mathrm{H}$ & 2.729021000000 & -1.856289000000 & -1.303927000000 \\
\hline $\mathrm{H}$ & -3.283459000000 & 0.404117000000 & -0.000161000000 & $\mathrm{H}$ & 2.203067000000 & 0.268503000000 & -2.151077000000 \\
\hline $\mathrm{H}$ & -1.626662000000 & -3.513799000000 & 0.000980000000 & $\mathrm{H}$ & 1.904535000000 & -2.830832000000 & 0.719448000000 \\
\hline $\mathrm{H}$ & -0.302708000000 & -2.228482000000 & 0.000825000000 & $\mathrm{H}$ & 0.538311000000 & -1.631066000000 & 0.839685000000 \\
\hline $\mathrm{O}$ & -1.392114000000 & 2.098857000000 & -0.000228000000 & $\mathrm{~N}$ & 2.398122000000 & 0.642844000000 & 1.442947000000 \\
\hline $\mathrm{Li}$ & -0.908837000000 & 3.797895000000 & -0.001169000000 & C & 2.364785000000 & -0.514451000000 & 1.712066000000 \\
\hline C & 0.187530000000 & 0.344765000000 & -0.000015000000 & $\mathrm{H}$ & 2.604923000000 & -1.190282000000 & 2.526303000000 \\
\hline $\mathrm{C}$ & 0.877157000000 & 0.163410000000 & 1.204562000000 & 0 & 0.725559000000 & 2.046959000000 & -1.061476000000 \\
\hline $\mathrm{C}$ & 0.876649000000 & 0.161730000000 & -1.204639000000 & $\mathrm{Li}$ & 2.284882000000 & 2.117226000000 & 0.103248000000 \\
\hline $\mathrm{C}$ & 2.224615000000 & -0.197725000000 & 1.205820000000 & $\mathrm{C}$ & -0.783079000000 & 0.343670000000 & -0.405693000000 \\
\hline C & 2.224114000000 & -0.199389000000 & -1.205970000000 & $\mathrm{C}$ & -1.433159000000 & -0.786937000000 & -0.917810000000 \\
\hline C & 2.902460000000 & -0.381215000000 & -0.000090000000 & $\mathrm{C}$ & -1.426799000000 & 1.109288000000 & 0.575515000000 \\
\hline $\mathrm{H}$ & 0.352335000000 & 0.296166000000 & 2.144864000000 & $\mathrm{C}$ & -2.694822000000 & -1.149186000000 & -0.451841000000 \\
\hline $\mathrm{H}$ & 0.351402000000 & 0.293130000000 & -2.144892000000 & $\mathrm{C}$ & -2.678741000000 & 0.736051000000 & 1.057281000000 \\
\hline $\mathrm{H}$ & 2.743956000000 & -0.339816000000 & 2.147580000000 & $\mathrm{C}$ & -3.317057000000 & -0.393799000000 & 0.542998000000 \\
\hline $\mathrm{H}$ & 2.743063000000 & -0.342758000000 & -2.147752000000 & $\mathrm{H}$ & -0.949118000000 & -1.374107000000 & -1.688789000000 \\
\hline \multirow[t]{4}{*}{$\mathrm{H}$} & 3.948932000000 & -0.665394000000 & -0.000106000000 & $\mathrm{H}$ & -0.934618000000 & 1.993624000000 & 0.961474000000 \\
\hline & & & & $\mathrm{H}$ & -3.193319000000 & -2.018794000000 & -0.865332000000 \\
\hline & & & & $\mathrm{H}$ & -3.159136000000 & 1.326864000000 & 1.829286000000 \\
\hline & & & & $\mathrm{H}$ & -4.295869000000 & -0.680318000000 & 0.911322000000 \\
\hline \multicolumn{4}{|c|}{ Product of exo-1 path } & & & & \\
\hline \multicolumn{4}{|c|}{$E_{\text {tot. }}=-562.89155$ a.u., $v_{\text {im. }}=0(-562.73023)$} & & & & \\
\hline C & -2.314555000000 & -0.813224000000 & 1.265149000000 & & & & \\
\hline $\mathrm{C}$ & -1.344473000000 & 0.093528000000 & 1.364876000000 & & & & \\
\hline C & -2.820513000000 & -1.274030000000 & -0.069492000000 & & & & \\
\hline $\mathrm{C}$ & -0.669207000000 & 0.758914000000 & 0.174858000000 & & & & \\
\hline $\mathrm{H}$ & -2.780872000000 & -1.233353000000 & 2.151755000000 & & & & \\
\hline $\mathrm{H}$ & -0.992902000000 & 0.438685000000 & 2.332610000000 & & & & \\
\hline $\mathrm{H}$ & -3.917473000000 & -1.252645000000 & -0.106217000000 & & & & \\
\hline $\mathrm{H}$ & -2.559410000000 & -2.325615000000 & -0.260377000000 & & & & \\
\hline $\mathrm{N}$ & -1.358235000000 & 0.427727000000 & -1.134534000000 & & & & \\
\hline $\mathrm{C}$ & -2.295232000000 & -0.429780000000 & -1.197236000000 & & & & \\
\hline $\mathrm{H}$ & -2.792259000000 & -0.556890000000 & -2.163374000000 & & & & \\
\hline 0 & -0.707638000000 & 2.118799000000 & 0.278850000000 & & & & \\
\hline $\mathrm{Li}$ & -1.152130000000 & 2.527168000000 & -1.460626000000 & & & & \\
\hline $\mathrm{C}$ & 0.775497000000 & 0.197589000000 & 0.055920000000 & & & & \\
\hline C & 1.030191000000 & -1.154376000000 & -0.207119000000 & & & & \\
\hline $\mathrm{C}$ & 1.859974000000 & 1.056430000000 & 0.240926000000 & & & & \\
\hline C & 2.336224000000 & -1.633155000000 & -0.290402000000 & & & & \\
\hline C & 3.171372000000 & 0.580889000000 & 0.164177000000 & & & & \\
\hline C & 3.414681000000 & -0.765114000000 & -0.103756000000 & & & & \\
\hline $\mathrm{H}$ & 0.204506000000 & -1.843743000000 & -0.347238000000 & & & & \\
\hline $\mathrm{H}$ & 1.651591000000 & 2.099073000000 & 0.447354000000 & & & & \\
\hline $\mathrm{H}$ & 2.514239000000 & -2.682959000000 & -0.498693000000 & & & & \\
\hline $\mathrm{H}$ & 4.002306000000 & 1.262504000000 & 0.314419000000 & & & & \\
\hline $\mathrm{H}$ & 4.431652000000 & -1.137061000000 & -0.164823000000 & & & & \\
\hline
\end{tabular}


Table S6. Optimized geometries of stationary points of interaction of lithium 1-phenylbuta-1,3-dien-1-olate $\mathbf{2 0}$ and HCN. PCM/MP2//HF/6-311++G(d,p) calculations $\left(\mathrm{THF},-78^{\circ} \mathrm{C}\right)$. Coordinates are in angstroms. Sum of electronic and thermal Free Energies in a.u. are in parentheses.

\begin{tabular}{|c|c|c|c|c|c|c|c|}
\hline \multicolumn{4}{|c|}{$\begin{array}{c}20 \\
E_{\text {tot. }}=-467.98615 \text { a.u., } v_{\text {im. }}=0(-467.83813)\end{array}$} & \multicolumn{4}{|c|}{$\begin{array}{c}\text { Product } \\
E_{\text {tot. }}=-561.22225 \text { a.u., } v_{\text {im. }}=0(-561.04712)\end{array}$} \\
\hline C & -2.333908000000 & -1.496453000000 & -0.070463000000 & $\mathrm{C}$ & -2.299290000000 & -0.786666000000 & 1.284155000000 \\
\hline C & -2.286005000000 & -0.037406000000 & 0.041373000000 & $\mathrm{C}$ & -1.335417000000 & 0.109400000000 & 1.355352000000 \\
\hline C & -1.418996000000 & -2.412992000000 & -0.393293000000 & $\mathrm{C}$ & -2.826854000000 & -1.260266000000 & -0.036797000000 \\
\hline $\mathrm{C}$ & -1.230460000000 & 0.818298000000 & 0.063227000000 & $\mathrm{C}$ & -0.671356000000 & 0.750911000000 & 0.148470000000 \\
\hline $\mathrm{H}$ & -3.322903000000 & -1.885064000000 & 0.130527000000 & $\mathrm{H}$ & -2.742813000000 & -1.197173000000 & 2.176169000000 \\
\hline $\mathrm{H}$ & -3.252450000000 & 0.436575000000 & 0.111899000000 & $\mathrm{H}$ & -0.967681000000 & 0.455255000000 & 2.307029000000 \\
\hline $\mathrm{H}$ & -1.695184000000 & -3.453533000000 & -0.417940000000 & $\mathrm{H}$ & -3.912978000000 & -1.201263000000 & -0.067283000000 \\
\hline $\mathrm{H}$ & -0.399752000000 & -2.177437000000 & -0.635663000000 & $\mathrm{H}$ & -2.588584000000 & -2.309683000000 & -0.209331000000 \\
\hline $\mathrm{O}$ & -1.357341000000 & 2.092960000000 & 0.117855000000 & $\mathrm{~N}$ & -1.360066000000 & 0.395132000000 & -1.125832000000 \\
\hline $\mathrm{Li}$ & -0.872395000000 & 3.779996000000 & 0.211196000000 & $\mathrm{C}$ & -2.284198000000 & -0.447315000000 & -1.177835000000 \\
\hline C & 0.200213000000 & 0.341804000000 & 0.043599000000 & $\mathrm{H}$ & -2.761035000000 & -0.606101000000 & -2.137955000000 \\
\hline C & 0.795850000000 & -0.172193000000 & 1.188052000000 & 0 & -0.702441000000 & 2.095309000000 & 0.234292000000 \\
\hline C & 0.966007000000 & 0.478898000000 & -1.108843000000 & $\mathrm{Li}$ & -1.158753000000 & 2.518388000000 & -1.480134000000 \\
\hline $\mathrm{C}$ & 2.131023000000 & -0.548409000000 & 1.180633000000 & $\mathrm{C}$ & 0.773329000000 & 0.200607000000 & 0.042498000000 \\
\hline C & 2.297834000000 & 0.094224000000 & -1.121683000000 & $\mathrm{C}$ & 1.035145000000 & -1.139605000000 & -0.238119000000 \\
\hline C & 2.885715000000 & -0.420136000000 & 0.024821000000 & $\mathrm{C}$ & 1.850466000000 & 1.047214000000 & 0.259590000000 \\
\hline $\mathrm{H}$ & 0.212969000000 & -0.286947000000 & 2.084860000000 & $\mathrm{C}$ & 2.332919000000 & -1.615287000000 & -0.307279000000 \\
\hline $\mathrm{H}$ & 0.516381000000 & 0.876346000000 & -2.002147000000 & $\mathrm{C}$ & 3.156445000000 & 0.573558000000 & 0.194656000000 \\
\hline $\mathrm{H}$ & 2.578905000000 & -0.944967000000 & 2.074945000000 & $\mathrm{C}$ & 3.403811000000 & -0.757407000000 & -0.090452000000 \\
\hline $\mathrm{H}$ & 2.875004000000 & 0.195265000000 & -2.024107000000 & $\mathrm{H}$ & 0.222018000000 & -1.823358000000 & -0.404703000000 \\
\hline \multirow[t]{4}{*}{$\mathrm{H}$} & 3.919304000000 & -0.717713000000 & 0.016667000000 & $\mathrm{H}$ & 1.656665000000 & 2.078642000000 & 0.480363000000 \\
\hline & & & & $\mathrm{H}$ & 2.510476000000 & -2.653383000000 & -0.528519000000 \\
\hline & & & & $\mathrm{H}$ & 3.976899000000 & 1.248396000000 & 0.367806000000 \\
\hline & & & & $\mathrm{H}$ & 4.413285000000 & -1.125764000000 & -0.142127000000 \\
\hline
\end{tabular}


Table S7. Optimized geometries of stationary points of interaction of lithium enolate 21 and HCN. PCM/B3LYP/6$311++\mathrm{G}(\mathrm{d}, \mathrm{p})$ calculations $\left(\mathrm{THF},-78^{\circ} \mathrm{C}\right)$. Coordinates are in angstroms. Sum of electronic and thermal Free Energies in a.u. are in parentheses.

\begin{tabular}{|c|c|c|c|c|c|c|c|}
\hline \multicolumn{4}{|c|}{$\begin{array}{c}\mathbf{2 1} \\
E_{\text {tot. }}=-467.98615 \text { a.u., } v_{\text {im. }}=0(-523.46786)\end{array}$} & \multicolumn{4}{|c|}{$\begin{array}{c}\text { TS1 of exo-1 path } \\
E_{\text {tot. }}=-617.04820 \text { a.u., } v_{\text {im. }}=-277.5 \mathrm{~cm}^{-1}(-616.89649)\end{array}$} \\
\hline $\mathrm{C}$ & -1.154844000000 & -1.562639000000 & -0.000168000000 & C & -0.857459000000 & -1.117081000000 & -0.657757000000 \\
\hline C & -1.103068000000 & -0.086112000000 & -0.002136000000 & C & -0.477695000000 & 0.271024000000 & -0.672462000000 \\
\hline C & -2.202511000000 & -2.425593000000 & -0.000660000000 & C & -2.133427000000 & -1.670750000000 & -0.739268000000 \\
\hline $\mathrm{C}$ & -2.204301000000 & 0.741525000000 & -0.002564000000 & $\mathrm{C}$ & -1.397879000000 & 1.310674000000 & -0.849786000000 \\
\hline $\mathrm{H}$ & -2.044192000000 & -3.497269000000 & 0.001544000000 & $\mathrm{H}$ & -2.238389000000 & -2.749704000000 & -0.674485000000 \\
\hline $\mathrm{H}$ & -3.222302000000 & -2.067605000000 & -0.003104000000 & $\mathrm{H}$ & -2.881421000000 & -1.167814000000 & -1.338548000000 \\
\hline $\mathrm{H}$ & -3.188023000000 & 0.247039000000 & -0.002344000000 & $\mathrm{H}$ & -2.351290000000 & 1.031743000000 & -1.324478000000 \\
\hline $\mathrm{O}$ & -2.197927000000 & 2.025041000000 & -0.002986000000 & $\mathrm{O}$ & -1.242890000000 & 2.523005000000 & -0.496997000000 \\
\hline $\mathrm{N}$ & 0.185898000000 & -1.976908000000 & 0.002822000000 & $\mathrm{~N}$ & 0.267524000000 & -1.832472000000 & -0.275006000000 \\
\hline $\mathrm{C}$ & 0.305863000000 & 0.293378000000 & -0.001614000000 & $\mathrm{C}$ & 0.912791000000 & 0.342139000000 & -0.252964000000 \\
\hline C & 0.978630000000 & 1.515520000000 & -0.003566000000 & C & 1.824895000000 & 1.387906000000 & -0.084602000000 \\
\hline C & 1.064928000000 & -0.905053000000 & 0.001459000000 & C & 1.345445000000 & -0.989382000000 & -0.035241000000 \\
\hline $\mathrm{C}$ & 2.453939000000 & -0.901098000000 & 0.002837000000 & $\mathrm{C}$ & 2.650246000000 & -1.295086000000 & 0.337513000000 \\
\hline $\mathrm{C}$ & 2.382692000000 & 1.531457000000 & -0.002363000000 & $\mathrm{C}$ & 3.137622000000 & 1.090897000000 & 0.293330000000 \\
\hline C & 3.108957000000 & 0.340473000000 & 0.000874000000 & C & 3.544639000000 & -0.232614000000 & 0.501743000000 \\
\hline $\mathrm{H}$ & 0.417010000000 & 2.440576000000 & -0.006173000000 & $\mathrm{H}$ & 1.513519000000 & 2.412147000000 & -0.247651000000 \\
\hline $\mathrm{H}$ & 2.907654000000 & 2.480686000000 & -0.003971000000 & $\mathrm{H}$ & 3.851621000000 & 1.896292000000 & 0.425758000000 \\
\hline $\mathrm{H}$ & 4.193227000000 & 0.368254000000 & 0.001818000000 & $\mathrm{H}$ & 4.568055000000 & -0.440446000000 & 0.793600000000 \\
\hline $\mathrm{H}$ & 3.018061000000 & -1.827563000000 & 0.005304000000 & $\mathrm{H}$ & 2.965851000000 & -2.320280000000 & 0.496305000000 \\
\hline $\mathrm{H}$ & 0.466185000000 & -2.944082000000 & 0.002927000000 & $\mathrm{H}$ & 0.289993000000 & -2.835727000000 & -0.182140000000 \\
\hline \multirow[t]{4}{*}{$\mathrm{Li}$} & -2.682401000000 & 3.728945000000 & 0.018516000000 & $\mathrm{H}$ & -3.586207000000 & -1.727216000000 & 1.370387000000 \\
\hline & & & & $\mathrm{C}$ & -3.091010000000 & -0.813211000000 & 1.083191000000 \\
\hline & & & & $\mathrm{N}$ & -2.924716000000 & 0.328179000000 & 1.324840000000 \\
\hline & & & & $\mathrm{Li}$ & -2.112419000000 & 2.179977000000 & 1.255403000000 \\
\hline \multirow{2}{*}{\multicolumn{4}{|c|}{$\begin{array}{c}\text { Intermediate } 21^{\prime} \text { of exo-1 path } \\
\mathbf{E}_{\text {tot. }}=-617.06116 \text { a.u., } v_{\text {im. }}=0(-616.90672)\end{array}$}} & \multicolumn{4}{|c|}{ TS2 of exo-1 path } \\
\hline & & & & & $E_{\text {tot. }}=-617.06111 \mathrm{a}$ & u., $v_{\text {im. }}=-73.7 \mathrm{~cm}$ & $1(-616.90545)$ \\
\hline$C$ & -0.800004000000 & -1.085564000000 & -0.478497000000 & C & -0.782079000000 & -1.119253000000 & -0.428185000000 \\
\hline $\mathrm{C}$ & -0.459100000000 & 0.264617000000 & -0.512745000000 & C & -0.469935000000 & 0.232792000000 & -0.472624000000 \\
\hline $\mathrm{C}$ & -2.165293000000 & -1.638310000000 & -0.459056000000 & C & -2.141926000000 & -1.692576000000 & -0.405342000000 \\
\hline C & -1.447635000000 & 1.293466000000 & -0.689336000000 & C & -1.482210000000 & 1.250735000000 & -0.656772000000 \\
\hline $\mathrm{H}$ & -2.159006000000 & -2.717289000000 & -0.285476000000 & $\mathrm{H}$ & -2.126590000000 & -2.753974000000 & -0.144254000000 \\
\hline $\mathrm{H}$ & -2.683688000000 & -1.465451000000 & -1.408612000000 & $\mathrm{H}$ & -2.632350000000 & -1.610202000000 & -1.382592000000 \\
\hline $\mathrm{H}$ & -2.301442000000 & 1.025390000000 & -1.325762000000 & $\mathrm{H}$ & -2.279958000000 & 0.997501000000 & -1.367523000000 \\
\hline 0 & -1.321199000000 & 2.486314000000 & -0.306327000000 & 0 & -1.356728000000 & 2.450727000000 & -0.278752000000 \\
\hline $\mathrm{N}$ & 0.324110000000 & -1.813547000000 & -0.168772000000 & $\mathrm{~N}$ & 0.359739000000 & -1.829174000000 & -0.131478000000 \\
\hline $\mathrm{C}$ & 0.949146000000 & 0.361417000000 & -0.192753000000 & $\mathrm{C}$ & 0.940687000000 & 0.357476000000 & -0.178698000000 \\
\hline $\mathrm{C}$ & 1.861049000000 & 1.420321000000 & -0.075992000000 & C & 1.836901000000 & 1.433334000000 & -0.087127000000 \\
\hline $\mathrm{C}$ & 1.411581000000 & -0.967877000000 & -0.009979000000 & C & 1.431809000000 & -0.962916000000 & 0.004653000000 \\
\hline $\mathrm{C}$ & 2.747462000000 & -1.262570000000 & 0.265252000000 & $\mathrm{C}$ & 2.778156000000 & -1.230405000000 & 0.258621000000 \\
\hline $\mathrm{C}$ & 3.191764000000 & 1.133174000000 & 0.210581000000 & $\mathrm{C}$ & 3.177357000000 & 1.172393000000 & 0.175808000000 \\
\hline $\mathrm{C}$ & 3.630889000000 & -0.193073000000 & 0.376833000000 & C & 3.643869000000 & -0.144824000000 & 0.345262000000 \\
\hline $\mathrm{H}$ & 1.528803000000 & 2.443332000000 & -0.202258000000 & $\mathrm{H}$ & 1.484346000000 & 2.449245000000 & -0.216155000000 \\
\hline $\mathrm{H}$ & 3.905360000000 & 1.943822000000 & 0.307437000000 & $\mathrm{H}$ & 3.878291000000 & 1.996360000000 & 0.251115000000 \\
\hline $\mathrm{H}$ & 4.674761000000 & -0.386344000000 & 0.596709000000 & $\mathrm{H}$ & 4.695045000000 & -0.317375000000 & 0.547089000000 \\
\hline $\mathrm{H}$ & 3.085661000000 & -2.284908000000 & 0.391454000000 & $\mathrm{H}$ & 3.136907000000 & -2.245633000000 & 0.386543000000 \\
\hline $\mathrm{H}$ & 0.348441000000 & -2.817543000000 & -0.075568000000 & $\mathrm{H}$ & 0.405147000000 & -2.832141000000 & -0.037459000000 \\
\hline $\mathrm{H}$ & -3.751833000000 & -1.679394000000 & 1.102161000000 & $\mathrm{H}$ & -3.795571000000 & -1.604286000000 & 1.081486000000 \\
\hline$C$ & -3.075482000000 & -0.927559000000 & 0.646397000000 & C & -3.079699000000 & -0.907848000000 & 0.601684000000 \\
\hline $\mathrm{N}$ & -3.070735000000 & 0.276839000000 & 0.936546000000 & $\mathrm{~N}$ & -3.056688000000 & 0.315105000000 & 0.814320000000 \\
\hline $\mathrm{Li}$ & -2.639121000000 & 2.138859000000 & 1.163963000000 & $\mathrm{Li}$ & -2.716797000000 & 2.193244000000 & 1.129401000000 \\
\hline
\end{tabular}




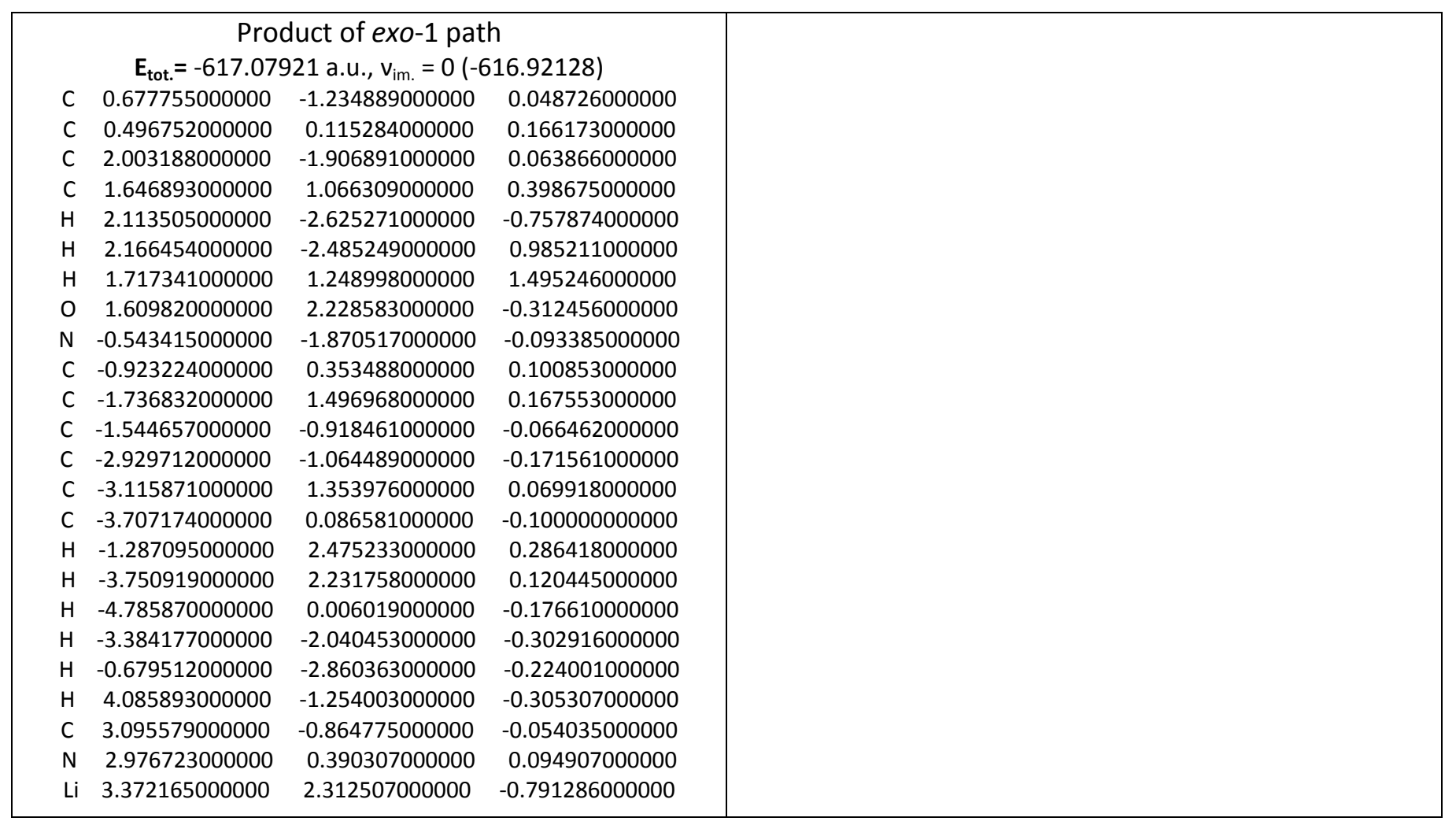

Table S8. Optimized geometries of stationary points of interaction of lithium enolate 21 and HCN. PCM/MP2//HF/6$311++\mathrm{G}(\mathrm{d}, \mathrm{p})$ calculations $\left(\mathrm{THF},-78^{\circ} \mathrm{C}\right)$. Coordinates are in angstroms. Sum of electronic and thermal Free Energies in a.u. are in parentheses.

\begin{tabular}{|c|c|c|c|c|c|c|c|}
\hline \multicolumn{4}{|c|}{$\begin{array}{c}\mathbf{2 1} \\
E_{\text {tot. }}=-522.04314 \text { a.u., } v_{\text {im. }}=0(-521.89904)\end{array}$} & \multicolumn{4}{|c|}{$\begin{array}{c}\text { Product of exo-1 path } \\
E_{\text {tot. }}=-615.28452 \text { a.u., } v_{\text {im. }}=0(-615.11264)\end{array}$} \\
\hline C & -1.147910000000 & -1.554008000000 & -0.000197000000 & $\mathrm{C}$ & 0.668684000000 & -1.217916000000 & 0.030312000000 \\
\hline C & -1.103420000000 & -0.078759000000 & -0.002291000000 & $\mathrm{C}$ & 0.498850000000 & 0.109780000000 & 0.155696000000 \\
\hline $\mathrm{C}$ & -2.182868000000 & -2.413599000000 & -0.001117000000 & $\mathrm{C}$ & 1.992832000000 & -1.893422000000 & 0.045301000000 \\
\hline $\mathrm{C}$ & -2.194426000000 & 0.738141000000 & -0.002320000000 & $\mathrm{C}$ & 1.644061000000 & 1.062655000000 & 0.391874000000 \\
\hline $\mathrm{H}$ & -2.015086000000 & -3.476281000000 & 0.001296000000 & $\mathrm{H}$ & 2.117557000000 & -2.559788000000 & -0.805066000000 \\
\hline $\mathrm{H}$ & -3.197634000000 & -2.065343000000 & -0.004137000000 & $\mathrm{H}$ & 2.127142000000 & -2.505320000000 & 0.936195000000 \\
\hline $\mathrm{H}$ & -3.164522000000 & 0.243541000000 & -0.001887000000 & $\mathrm{H}$ & 1.673972000000 & 1.286590000000 & 1.467308000000 \\
\hline $\mathrm{O}$ & -2.197352000000 & 2.000929000000 & -0.002711000000 & $\mathrm{O}$ & 1.618684000000 & 2.189328000000 & -0.347974000000 \\
\hline $\mathrm{N}$ & 0.180608000000 & -1.959799000000 & 0.003318000000 & $\mathrm{~N}$ & -0.542674000000 & -1.854060000000 & -0.109062000000 \\
\hline C & 0.312135000000 & 0.300434000000 & -0.001759000000 & $\mathrm{C}$ & -0.927658000000 & 0.348250000000 & 0.099027000000 \\
\hline $\mathrm{C}$ & 0.982231000000 & 1.508116000000 & -0.003998000000 & $\mathrm{C}$ & -1.734147000000 & 1.487090000000 & 0.177662000000 \\
\hline $\mathrm{C}$ & 1.057839000000 & -0.890811000000 & 0.001642000000 & $\mathrm{C}$ & -1.534862000000 & -0.906654000000 & -0.071459000000 \\
\hline $\mathrm{C}$ & 2.433894000000 & -0.896991000000 & 0.003060000000 & $\mathrm{C}$ & -2.916263000000 & -1.054398000000 & -0.171307000000 \\
\hline C & 2.381750000000 & 1.518820000000 & -0.002738000000 & $\mathrm{C}$ & -3.103084000000 & 1.347681000000 & 0.086400000000 \\
\hline $\mathrm{C}$ & 3.094589000000 & 0.335172000000 & 0.000800000000 & $\mathrm{C}$ & -3.688426000000 & 0.084725000000 & -0.089178000000 \\
\hline $\mathrm{H}$ & 0.434751000000 & 2.431039000000 & -0.006876000000 & $\mathrm{H}$ & -1.288398000000 & 2.456593000000 & 0.302024000000 \\
\hline $\mathrm{H}$ & 2.905438000000 & 2.458792000000 & -0.004578000000 & $\mathrm{H}$ & -3.734326000000 & 2.216696000000 & 0.145700000000 \\
\hline $\mathrm{H}$ & 4.170379000000 & 0.356025000000 & 0.001770000000 & $\mathrm{H}$ & -4.758837000000 & 0.003981000000 & -0.161294000000 \\
\hline $\mathrm{H}$ & 2.987833000000 & -1.819437000000 & 0.005792000000 & $\mathrm{H}$ & -3.365421000000 & -2.022534000000 & -0.305798000000 \\
\hline $\mathrm{H}$ & 0.459087000000 & -2.911950000000 & 0.003128000000 & $\mathrm{H}$ & -0.675493000000 & -2.827496000000 & -0.249536000000 \\
\hline \multirow[t]{4}{*}{$\mathrm{Li}$} & -2.689523000000 & 3.698560000000 & 0.019153000000 & $\mathrm{H}$ & 4.077831000000 & -1.232572000000 & -0.193427000000 \\
\hline & & & & $\mathrm{C}$ & 3.083350000000 & -0.848964000000 & -0.000889000000 \\
\hline & & & & $\mathrm{N}$ & 2.954232000000 & 0.386605000000 & 0.143815000000 \\
\hline & & & & $\mathrm{Li}$ & 3.365189000000 & 2.276153000000 & -0.838742000000 \\
\hline
\end{tabular}


Table S9. Optimized geometries of stationary points of interaction of lithium enolate $\mathbf{1 5}$ and benzonitrile. $\mathrm{PCM} / \mathrm{B} 3 \mathrm{LYP} / 6-311++\mathrm{G}(\mathrm{d}, \mathrm{p})$ calculations $\left(\mathrm{THF},-78^{\circ} \mathrm{C}\right)$. Coordinates are in angstroms. Sum of electronic and thermal Free Energies are in parentheses.

\begin{tabular}{|c|c|c|c|c|c|c|c|}
\hline \multicolumn{4}{|c|}{$\begin{array}{c}15 \\
E_{\text {tot. }}=-794.02484 \text { a.u., } v_{\text {im. }}=0(-793.78800)\end{array}$} & \multicolumn{4}{|c|}{$\begin{array}{c}\text { TS1 of exo-1 path } \\
E_{\text {tot. }}=-1118.59185 \text { a.u., } v_{\text {im. }}=-273.5 \mathrm{~cm}^{-1}(-1118.25793)\end{array}$} \\
\hline C & 0.400929000000 & 1.345748000000 & -0.037051000000 & C & -0.573522000000 & -0.429333000000 & 0.886750000000 \\
\hline C & 0.344819000000 & -0.133395000000 & -0.016993000000 & C & -1.528488000000 & 0.130737000000 & -0.019610000000 \\
\hline C & -0.568486000000 & 2.298133000000 & -0.088371000000 & $\mathrm{C}$ & 0.764439000000 & -0.043241000000 & 1.082560000000 \\
\hline C & -0.764859000000 & -0.963043000000 & -0.012045000000 & C & -1.421582000000 & 1.350971000000 & -0.756758000000 \\
\hline $\mathrm{H}$ & -0.317612000000 & 3.349887000000 & -0.104511000000 & $\mathrm{H}$ & 1.352976000000 & -0.588036000000 & 1.810835000000 \\
\hline $\mathrm{H}$ & -1.612543000000 & 2.035314000000 & -0.113457000000 & $\mathrm{H}$ & 0.986753000000 & 1.013993000000 & 1.030650000000 \\
\hline $\mathrm{O}$ & -0.680975000000 & -2.252245000000 & -0.013261000000 & $\mathrm{O}$ & -1.877839000000 & 1.443356000000 & -1.935573000000 \\
\hline $\mathrm{N}$ & 1.768683000000 & 1.680950000000 & -0.004759000000 & $\mathrm{~N}$ & -1.063589000000 & -1.664005000000 & 1.301466000000 \\
\hline C & 1.729251000000 & -0.600278000000 & -0.002571000000 & C & -2.573224000000 & -0.861023000000 & -0.199874000000 \\
\hline C & 2.318297000000 & -1.865850000000 & 0.008771000000 & C & -3.755452000000 & -0.903144000000 & -0.948754000000 \\
\hline C & 2.566837000000 & 0.544577000000 & 0.003783000000 & C & -2.261652000000 & -1.947794000000 & 0.655901000000 \\
\hline C & 3.954433000000 & 0.438577000000 & 0.018672000000 & C & -3.094609000000 & -3.059799000000 & 0.769644000000 \\
\hline C & 3.717036000000 & -1.981587000000 & 0.025081000000 & C & -4.593330000000 & -2.014308000000 & -0.839055000000 \\
\hline C & 4.522840000000 & -0.844276000000 & 0.029754000000 & C & -4.265962000000 & -3.080772000000 & 0.008488000000 \\
\hline $\mathrm{H}$ & 1.690145000000 & -2.745157000000 & 0.003498000000 & $\mathrm{H}$ & -4.008979000000 & -0.084086000000 & -1.610329000000 \\
\hline $\mathrm{H}$ & 4.173493000000 & -2.965681000000 & 0.032751000000 & $\mathrm{H}$ & -5.507240000000 & -2.055219000000 & -1.421456000000 \\
\hline $\mathrm{H}$ & 5.602896000000 & -0.944238000000 & 0.040840000000 & $\mathrm{H}$ & -4.926013000000 & -3.938486000000 & 0.074364000000 \\
\hline $\mathrm{H}$ & 4.592012000000 & 1.314556000000 & 0.020777000000 & $\mathrm{H}$ & -2.849064000000 & -3.890989000000 & 1.419660000000 \\
\hline $\mathrm{Li}$ & -1.435272000000 & -3.885782000000 & -0.011374000000 & C & 1.622299000000 & -0.455185000000 & -0.839442000000 \\
\hline C & 2.246848000000 & 3.043648000000 & -0.025189000000 & $\mathrm{~N}$ & 0.920474000000 & -0.292665000000 & -1.788514000000 \\
\hline $\mathrm{H}$ & 1.838041000000 & 3.612374000000 & 0.816674000000 & $\mathrm{Li}$ & -0.757136000000 & 0.065053000000 & -2.771338000000 \\
\hline $\mathrm{H}$ & 3.332157000000 & 3.053620000000 & 0.051080000000 & C & -0.353546000000 & -2.586679000000 & 2.167314000000 \\
\hline $\mathrm{H}$ & 1.959594000000 & 3.551707000000 & -0.953099000000 & $\mathrm{H}$ & 0.133416000000 & -2.038763000000 & 2.974756000000 \\
\hline C & -2.174255000000 & -0.430765000000 & 0.003022000000 & $\mathrm{H}$ & -1.062211000000 & -3.284367000000 & 2.611604000000 \\
\hline C & -2.817793000000 & -0.159142000000 & 1.215934000000 & $\mathrm{H}$ & 0.404946000000 & -3.156311000000 & 1.619892000000 \\
\hline C & -2.892600000000 & -0.289549000000 & -1.189982000000 & C & 2.992145000000 & -0.879921000000 & -0.511611000000 \\
\hline C & -4.151546000000 & 0.248714000000 & 1.236097000000 & C & 3.497103000000 & -2.044211000000 & -1.109451000000 \\
\hline C & -4.225518000000 & 0.121410000000 & -1.171756000000 & C & 3.812535000000 & -0.142184000000 & 0.350580000000 \\
\hline C & -4.858953000000 & 0.391937000000 & 0.041840000000 & C & 4.797396000000 & -2.464958000000 & -0.839420000000 \\
\hline $\mathrm{H}$ & -2.268305000000 & -0.258633000000 & 2.146064000000 & $\mathrm{C}$ & 5.119391000000 & -0.554538000000 & 0.598861000000 \\
\hline $\mathrm{H}$ & -2.402114000000 & -0.491511000000 & -2.136432000000 & C & 5.613799000000 & -1.720162000000 & 0.011814000000 \\
\hline $\mathrm{H}$ & -4.636592000000 & 0.459218000000 & 2.183193000000 & $\mathrm{H}$ & 2.865533000000 & -2.613938000000 & -1.780718000000 \\
\hline $\mathrm{H}$ & -4.767779000000 & 0.234058000000 & -2.104418000000 & $\mathrm{H}$ & 3.431470000000 & 0.757281000000 & 0.817746000000 \\
\hline $\mathrm{H}$ & -5.894257000000 & 0.713980000000 & 0.056572000000 & $\mathrm{H}$ & 5.174364000000 & -3.371610000000 & -1.299480000000 \\
\hline & & & & $\mathrm{H}$ & 5.751139000000 & 0.032795000000 & 1.255662000000 \\
\hline & & & & $\mathrm{H}$ & 6.627554000000 & -2.045042000000 & 0.216525000000 \\
\hline & & & & C & -0.828223000000 & 2.584679000000 & -0.149304000000 \\
\hline & & & & $\mathrm{C}$ & -0.998405000000 & 2.883512000000 & 1.209331000000 \\
\hline & & & & C & -0.168932000000 & 3.509952000000 & -0.970103000000 \\
\hline & & & & C & -0.510004000000 & 4.076371000000 & 1.737375000000 \\
\hline & & & & $\mathrm{C}$ & 0.335574000000 & 4.693723000000 & -0.439454000000 \\
\hline & & & & $\mathrm{C}$ & 0.164529000000 & 4.980674000000 & 0.916209000000 \\
\hline & & & & $\mathrm{H}$ & -1.526126000000 & 2.183643000000 & 1.845922000000 \\
\hline & & & & $\mathrm{H}$ & -0.053159000000 & 3.288960000000 & -2.024127000000 \\
\hline & & & & $\mathrm{H}$ & -0.659109000000 & 4.302508000000 & 2.787258000000 \\
\hline & & & & $\mathrm{H}$ & 0.856953000000 & 5.395170000000 & -1.081208000000 \\
\hline & & & & $\mathrm{H}$ & 0.549048000000 & 5.906977000000 & 1.328434000000 \\
\hline
\end{tabular}




\begin{tabular}{|c|c|c|c|c|c|c|c|}
\hline \multicolumn{4}{|c|}{$\begin{array}{c}\text { Intermediate } 15^{`} \text { of exo-1 path } \\
\mathbf{E}_{\text {tot. }}= \\
-1118.60099 \text { a.u., } v_{\text {im. }}=0(-1118.26445)\end{array}$} & \multicolumn{4}{|c|}{$\begin{array}{c}\text { TS2 of exo-1 path } \\
E_{\text {tot. }}=-1118.60045 \text { a.u., } v_{\text {im. }}=-66.6 \mathrm{~cm}^{-1}(-1118.25886)\end{array}$} \\
\hline C & 0.723936000000 & -0.593079000000 & -0.700866000000 & C & 0.779583000000 & -0.755276000000 & -0.650002000000 \\
\hline $\mathrm{C}$ & 1.552044000000 & 0.196800000000 & 0.100309000000 & C & 1.458766000000 & 0.112326000000 & 0.190637000000 \\
\hline C & -0.734237000000 & -0.463701000000 & -0.886061000000 & C & -0.671645000000 & -0.746357000000 & -0.953806000000 \\
\hline $\mathrm{C}$ & 1.170461000000 & 1.406861000000 & 0.814719000000 & $\mathrm{C}$ & 0.886818000000 & 1.284386000000 & 0.870478000000 \\
\hline $\mathrm{H}$ & -1.107971000000 & -1.213904000000 & -1.581441000000 & $\mathrm{H}$ & -0.975583000000 & -1.683956000000 & -1.418505000000 \\
\hline $\mathrm{H}$ & -0.988943000000 & 0.514777000000 & -1.300043000000 & $\mathrm{H}$ & -0.921150000000 & 0.051857000000 & -1.662709000000 \\
\hline 0 & 1.547772000000 & 1.597019000000 & 1.996101000000 & 0 & 1.253652000000 & 1.572441000000 & 2.050248000000 \\
\hline $\mathrm{N}$ & 1.420011000000 & -1.727468000000 & -1.075281000000 & $\mathrm{~N}$ & 1.629445000000 & -1.782318000000 & -1.033117000000 \\
\hline $\mathrm{C}$ & 2.804435000000 & -0.508319000000 & 0.247841000000 & $\mathrm{C}$ & 2.789122000000 & -0.416352000000 & 0.361998000000 \\
\hline $\mathrm{C}$ & 4.008802000000 & -0.233818000000 & 0.912329000000 & $\mathrm{C}$ & 3.924797000000 & -0.001171000000 & 1.075607000000 \\
\hline C & 2.692410000000 & -1.694182000000 & -0.520736000000 & C & 2.861685000000 & -1.594919000000 & -0.428296000000 \\
\hline $\mathrm{C}$ & 3.750119000000 & -2.600391000000 & -0.640061000000 & $\mathrm{C}$ & 4.032746000000 & -2.355167000000 & -0.515401000000 \\
\hline C & 5.060863000000 & -1.135877000000 & 0.800744000000 & C & 5.088294000000 & -0.756160000000 & 0.991460000000 \\
\hline $\mathrm{C}$ & 4.932447000000 & -2.306949000000 & 0.032163000000 & $\mathrm{C}$ & 5.141538000000 & -1.921832000000 & 0.203723000000 \\
\hline $\mathrm{H}$ & 4.110494000000 & 0.663338000000 & 1.510857000000 & $\mathrm{H}$ & 3.884218000000 & 0.890688000000 & 1.688848000000 \\
\hline $\mathrm{H}$ & 5.994278000000 & -0.937607000000 & 1.315636000000 & $\mathrm{H}$ & 5.969764000000 & -0.446848000000 & 1.542252000000 \\
\hline $\mathrm{H}$ & 5.766421000000 & -2.996298000000 & -0.035965000000 & $\mathrm{H}$ & 6.060603000000 & -2.495361000000 & 0.158687000000 \\
\hline $\mathrm{H}$ & 3.661900000000 & -3.507639000000 & -1.225315000000 & $\mathrm{H}$ & 4.084039000000 & -3.257082000000 & -1.113620000000 \\
\hline $\mathrm{C}$ & -1.539534000000 & -0.565106000000 & 0.512934000000 & $\mathrm{C}$ & -1.540243000000 & -0.478726000000 & 0.336041000000 \\
\hline $\mathrm{N}$ & -1.067406000000 & -0.217612000000 & 1.608662000000 & $\mathrm{~N}$ & -1.125653000000 & 0.189829000000 & 1.307070000000 \\
\hline $\mathrm{Li}$ & 0.306999000000 & 0.280659000000 & 2.818403000000 & $\mathrm{Li}$ & -0.140133000000 & 0.575791000000 & 2.912459000000 \\
\hline C & 0.897335000000 & -2.831878000000 & -1.867563000000 & C & 1.283319000000 & -2.918176000000 & -1.874112000000 \\
\hline $\mathrm{H}$ & 0.269951000000 & -2.453265000000 & -2.673773000000 & $\mathrm{H}$ & 0.618955000000 & -2.602940000000 & -2.678328000000 \\
\hline $\mathrm{H}$ & 1.728794000000 & -3.373128000000 & -2.315680000000 & $\mathrm{H}$ & 2.189963000000 & -3.317489000000 & -2.326406000000 \\
\hline $\mathrm{H}$ & 0.314588000000 & -3.525403000000 & -1.254989000000 & $\mathrm{H}$ & 0.798016000000 & -3.713795000000 & -1.300724000000 \\
\hline $\mathrm{C}$ & -2.952680000000 & -1.085134000000 & 0.323589000000 & $\mathrm{C}$ & -2.930742000000 & -1.074392000000 & 0.278385000000 \\
\hline $\mathrm{C}$ & -3.576126000000 & -1.756101000000 & 1.386102000000 & $\mathrm{C}$ & -3.589367000000 & -1.400834000000 & 1.472754000000 \\
\hline C & -3.686583000000 & -0.890715000000 & -0.854978000000 & C & -3.609892000000 & -1.281241000000 & -0.930372000000 \\
\hline C & -4.881257000000 & -2.225479000000 & 1.274050000000 & C & -4.878244000000 & -1.924587000000 & 1.461622000000 \\
\hline C & -5.004270000000 & -1.340476000000 & -0.963189000000 & C & -4.910690000000 & -1.787676000000 & -0.943738000000 \\
\hline C & -5.605128000000 & -2.015347000000 & 0.097471000000 & C & -5.547982000000 & -2.116898000000 & 0.250773000000 \\
\hline $\mathrm{H}$ & -3.014917000000 & -1.895940000000 & 2.302837000000 & $\mathrm{H}$ & -3.071412000000 & -1.234690000000 & 2.410021000000 \\
\hline $\mathrm{H}$ & -3.243944000000 & -0.367291000000 & -1.694371000000 & $\mathrm{H}$ & -3.137618000000 & -1.030583000000 & -1.872983000000 \\
\hline $\mathrm{H}$ & -5.338163000000 & -2.753635000000 & 2.104423000000 & $\mathrm{H}$ & -5.364066000000 & -2.182115000000 & 2.396726000000 \\
\hline $\mathrm{H}$ & -5.559237000000 & -1.162669000000 & -1.878197000000 & $\mathrm{H}$ & -5.423780000000 & -1.924917000000 & -1.889411000000 \\
\hline $\mathrm{H}$ & -6.625017000000 & -2.373405000000 & 0.010716000000 & $\mathrm{H}$ & -6.555046000000 & -2.518614000000 & 0.240498000000 \\
\hline $\mathrm{C}$ & 0.433287000000 & 2.505265000000 & 0.116138000000 & C & 0.228455000000 & 2.369784000000 & 0.058952000000 \\
\hline C & 0.553822000000 & 2.703080000000 & -1.266030000000 & C & 0.393044000000 & 2.464411000000 & -1.328479000000 \\
\hline C & -0.321923000000 & 3.415113000000 & 0.869943000000 & C & -0.496048000000 & 3.375302000000 & 0.716794000000 \\
\hline C & -0.076752000000 & 3.781844000000 & -1.883006000000 & C & -0.168294000000 & 3.523062000000 & -2.043528000000 \\
\hline $\mathrm{C}$ & -0.964477000000 & 4.481978000000 & 0.252188000000 & $\mathrm{C}$ & -1.065035000000 & 4.425166000000 & 0.004770000000 \\
\hline C & -0.842301000000 & 4.668680000000 & -1.126984000000 & C & -0.904735000000 & 4.502926000000 & -1.381374000000 \\
\hline $\mathrm{H}$ & 1.157573000000 & 2.021244000000 & -1.852646000000 & $\mathrm{H}$ & 0.980938000000 & 1.718163000000 & -1.848961000000 \\
\hline $\mathrm{H}$ & -0.402804000000 & 3.272047000000 & 1.940171000000 & $\mathrm{H}$ & -0.608371000000 & 3.325233000000 & 1.792442000000 \\
\hline $\mathrm{H}$ & 0.033548000000 & 3.932156000000 & -2.950961000000 & $\mathrm{H}$ & -0.021876000000 & 3.584488000000 & -3.116375000000 \\
\hline $\mathrm{H}$ & -1.558319000000 & 5.170767000000 & 0.842292000000 & $\mathrm{H}$ & -1.630869000000 & 5.188079000000 & 0.528284000000 \\
\hline $\mathrm{H}$ & -1.337786000000 & 5.504865000000 & -1.607511000000 & $\mathrm{H}$ & -1.343604000000 & 5.324817000000 & -1.936080000000 \\
\hline
\end{tabular}




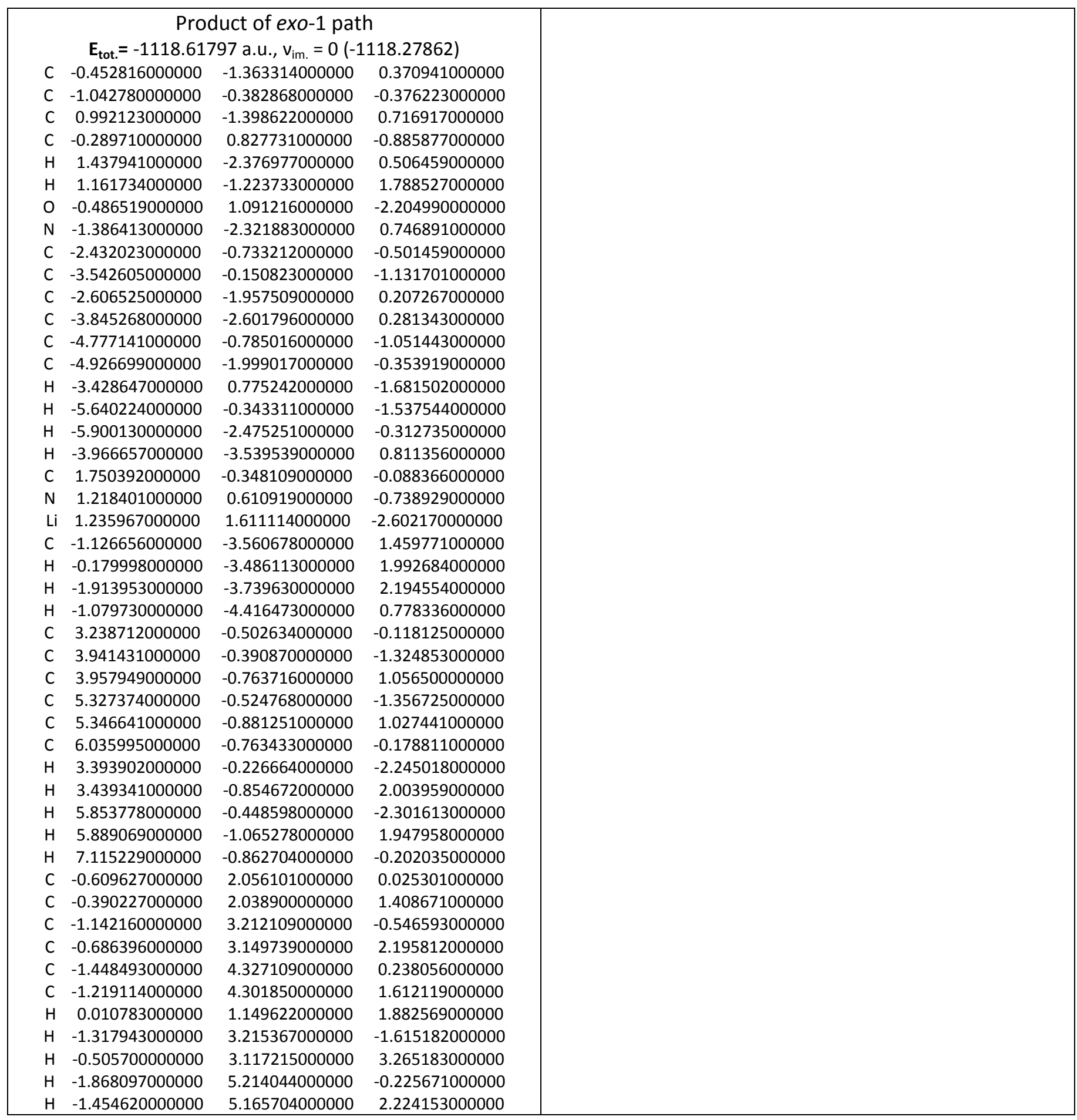


Table S10. Optimized geometries of stationary points of interaction of lithium enolate 15 and benzonitrile. $\mathrm{PCM} / \mathrm{MP} 2 / / \mathrm{HF} / 6-311++\mathrm{G}(\mathrm{d}, \mathrm{p})$ calculations $\left(\mathrm{THF},-78^{\circ} \mathrm{C}\right)$. Coordinates are in angstroms. Sum of electronic and thermal Free Energies in a.u. are in parentheses.

\begin{tabular}{|c|c|c|c|c|c|c|c|}
\hline \multicolumn{4}{|c|}{$\begin{array}{c}15 \\
E_{\text {tot. }}=-791.65569 \text { a.u., } v_{\text {im. }}=0(-791.40062)\end{array}$} & \multicolumn{4}{|c|}{$\begin{array}{c}\text { Product of exo-1 path } \\
E_{\text {tot. }}=-1115.32254 \text { a.u., } v_{\text {im. }}=0(-1114.95710)\end{array}$} \\
\hline C & 0.404580000000 & 1.348935000000 & -0.049912000000 & $\mathrm{C}$ & 0.464077000000 & -1.390371000000 & -0.334521000000 \\
\hline $\mathrm{C}$ & 0.333850000000 & -0.130923000000 & -0.019440000000 & $\mathrm{C}$ & 1.031718000000 & -0.397665000000 & 0.370059000000 \\
\hline C & -0.548417000000 & 2.296283000000 & -0.139723000000 & $\mathrm{C}$ & -0.987505000000 & -1.456809000000 & -0.652940000000 \\
\hline $\mathrm{C}$ & -0.760844000000 & -0.954288000000 & -0.009693000000 & $\mathrm{C}$ & 0.266446000000 & 0.800328000000 & 0.886899000000 \\
\hline $\mathrm{H}$ & -0.291522000000 & 3.337851000000 & -0.162588000000 & $\mathrm{H}$ & -1.415113000000 & -2.415767000000 & -0.375091000000 \\
\hline $\mathrm{H}$ & -1.585157000000 & 2.044372000000 & -0.190627000000 & $\mathrm{H}$ & -1.169582000000 & -1.341361000000 & -1.719458000000 \\
\hline $\mathrm{O}$ & -0.676860000000 & -2.221124000000 & -0.007784000000 & $\mathrm{O}$ & 0.478293000000 & 1.045402000000 & 2.191526000000 \\
\hline $\mathrm{N}$ & 1.760835000000 & 1.669243000000 & 0.016274000000 & $\mathrm{~N}$ & 1.400890000000 & -2.326242000000 & -0.724672000000 \\
\hline $\mathrm{C}$ & 1.723427000000 & -0.602830000000 & -0.001167000000 & $\mathrm{C}$ & 2.438005000000 & -0.713854000000 & 0.464015000000 \\
\hline $\mathrm{C}$ & 2.305146000000 & -1.856662000000 & 0.006829000000 & $\mathrm{C}$ & 3.541858000000 & -0.095681000000 & 1.054562000000 \\
\hline C & 2.552538000000 & 0.532940000000 & 0.013194000000 & $\mathrm{C}$ & 2.614680000000 & -1.926909000000 & -0.221819000000 \\
\hline C & 3.927932000000 & 0.428713000000 & 0.027458000000 & $\mathrm{C}$ & 3.861825000000 & -2.543755000000 & -0.315502000000 \\
\hline $\mathrm{C}$ & 3.699210000000 & -1.974718000000 & 0.024676000000 & $\mathrm{C}$ & 4.779990000000 & -0.696032000000 & 0.956111000000 \\
\hline C & 4.496021000000 & -0.848398000000 & 0.033660000000 & $\mathrm{C}$ & 4.935564000000 & -1.912767000000 & 0.277834000000 \\
\hline $\mathrm{H}$ & 1.689342000000 & -2.732645000000 & -0.002833000000 & $\mathrm{H}$ & 3.422775000000 & 0.830848000000 & 1.584607000000 \\
\hline $\mathrm{H}$ & 4.149559000000 & -2.952033000000 & 0.028964000000 & $\mathrm{H}$ & 5.638028000000 & -0.230774000000 & 1.408358000000 \\
\hline $\mathrm{H}$ & 5.567662000000 & -0.945434000000 & 0.044386000000 & $\mathrm{H}$ & 5.910285000000 & -2.364717000000 & 0.220169000000 \\
\hline $\mathrm{H}$ & 4.562809000000 & 1.294747000000 & 0.032726000000 & $\mathrm{H}$ & 3.991391000000 & -3.478727000000 & -0.829796000000 \\
\hline $\mathrm{Li}$ & -1.430519000000 & -3.849327000000 & -0.001374000000 & $\mathrm{C}$ & -1.735288000000 & -0.374973000000 & 0.109520000000 \\
\hline $\mathrm{C}$ & 2.250911000000 & 3.020935000000 & -0.037971000000 & $\mathrm{~N}$ & -1.210909000000 & 0.571432000000 & 0.740850000000 \\
\hline $\mathrm{H}$ & 1.796904000000 & 3.623012000000 & 0.741915000000 & $\mathrm{Li}$ & -1.213638000000 & 1.437356000000 & 2.722799000000 \\
\hline $\mathrm{H}$ & 3.319183000000 & 3.028804000000 & 0.114610000000 & $\mathrm{C}$ & 1.149029000000 & -3.592823000000 & -1.374060000000 \\
\hline $\mathrm{H}$ & 2.036673000000 & 3.483457000000 & -0.998670000000 & $\mathrm{H}$ & 0.183502000000 & -3.568295000000 & -1.858214000000 \\
\hline C & -2.172960000000 & -0.428269000000 & 0.008284000000 & $\mathrm{H}$ & 1.898306000000 & -3.774616000000 & -2.134683000000 \\
\hline C & -2.796950000000 & -0.127547000000 & 1.212039000000 & $\mathrm{H}$ & 1.162292000000 & -4.415621000000 & -0.664658000000 \\
\hline C & -2.896292000000 & -0.324900000000 & -1.173301000000 & $\mathrm{C}$ & -3.231928000000 & -0.504621000000 & 0.110159000000 \\
\hline $\mathrm{C}$ & -4.122492000000 & 0.279315000000 & 1.233978000000 & $\mathrm{C}$ & -3.939717000000 & -0.486877000000 & 1.306731000000 \\
\hline $\mathrm{C}$ & -4.220482000000 & 0.086105000000 & -1.153249000000 & $\mathrm{C}$ & -3.932722000000 & -0.649763000000 & -1.082234000000 \\
\hline C & -4.837548000000 & 0.389946000000 & 0.051040000000 & $\mathrm{C}$ & -5.320111000000 & -0.603828000000 & 1.311860000000 \\
\hline $\mathrm{H}$ & -2.244147000000 & -0.200347000000 & 2.132185000000 & $\mathrm{C}$ & -5.315536000000 & -0.751528000000 & -1.079152000000 \\
\hline $\mathrm{H}$ & -2.421348000000 & -0.553044000000 & -2.111555000000 & $\mathrm{C}$ & -6.013112000000 & -0.731198000000 & 0.117716000000 \\
\hline $\mathrm{H}$ & -4.593675000000 & 0.513653000000 & 2.172264000000 & $\mathrm{H}$ & -3.410401000000 & -0.399223000000 & 2.237527000000 \\
\hline $\mathrm{H}$ & -4.767461000000 & 0.171393000000 & -2.075678000000 & $\mathrm{H}$ & -3.409338000000 & -0.665554000000 & -2.021237000000 \\
\hline \multirow[t]{14}{*}{$\mathrm{H}$} & -5.864012000000 & 0.710656000000 & 0.067182000000 & $\mathrm{H}$ & -5.852356000000 & -0.597814000000 & 2.246358000000 \\
\hline & & & & $\mathrm{H}$ & -5.844234000000 & -0.846979000000 & -2.010653000000 \\
\hline & & & & $\mathrm{H}$ & -7.084924000000 & -0.817364000000 & 0.120784000000 \\
\hline & & & & $\mathrm{C}$ & 0.583775000000 & 2.039074000000 & 0.002192000000 \\
\hline & & & & $\mathrm{C}$ & 0.352187000000 & 2.044265000000 & -1.372908000000 \\
\hline & & & & $\mathrm{C}$ & 1.124390000000 & 3.179334000000 & 0.576423000000 \\
\hline & & & & $\mathrm{C}$ & 0.642866000000 & 3.155060000000 & -2.144460000000 \\
\hline & & & & $\mathrm{C}$ & 1.423019000000 & 4.298088000000 & -0.195009000000 \\
\hline & & & & $\mathrm{C}$ & 1.182165000000 & 4.292814000000 & -1.556607000000 \\
\hline & & & & $\mathrm{H}$ & -0.056019000000 & 1.173129000000 & -1.853102000000 \\
\hline & & & & $\mathrm{H}$ & 1.315915000000 & 3.182749000000 & 1.631314000000 \\
\hline & & & & $\mathrm{H}$ & 0.452603000000 & 3.134613000000 & -3.203523000000 \\
\hline & & & & $\mathrm{H}$ & 1.845517000000 & 5.170215000000 & 0.273596000000 \\
\hline & & & & $\mathrm{H}$ & 1.411995000000 & 5.156590000000 & -2.155363000000 \\
\hline
\end{tabular}


Table S11. Optimized geometries of stationary points of interaction of lithium enolate $\mathbf{1 5}$ and benzonitrile.

$\mathrm{PCM} / \mathrm{B} 3 \mathrm{LYP} / 6-311++\mathrm{G}(\mathrm{d}, \mathrm{p})$ calculations (Benzene, $25^{\circ} \mathrm{C}$ ). Coordinates are in angstroms. Sum of electronic and thermal Free Energies in a.u. are in parentheses.

\begin{tabular}{|c|c|c|c|c|c|c|c|}
\hline \multicolumn{4}{|c|}{$\begin{array}{c}15 \\
E_{\text {tot. }}=-794.00067 \text { a.u., } v_{\text {im. }}=0(-793.78377)\end{array}$} & \multicolumn{4}{|c|}{$\begin{array}{c}\text { TS1 of exo-1 path } \\
E_{\text {tot. }}=-1118.57956 \text { a.u., } v_{\text {im. }}=-251.2 \mathrm{~cm}^{-1}(-1118.26975)\end{array}$} \\
\hline C & 0.401591000000 & 1.348700000000 & -0.038391000000 & C & -0.512153000000 & -0.442127000000 & 0.879179000000 \\
\hline C & 0.343609000000 & -0.132956000000 & -0.017241000000 & C & -1.513297000000 & 0.019867000000 & -0.032790000000 \\
\hline $\mathrm{C}$ & -0.567120000000 & 2.297017000000 & -0.090041000000 & $\mathrm{C}$ & 0.790187000000 & 0.057770000000 & 1.056249000000 \\
\hline $\mathrm{C}$ & -0.765127000000 & -0.950543000000 & -0.011969000000 & C & -1.536310000000 & 1.247860000000 & -0.768887000000 \\
\hline $\mathrm{H}$ & -0.318710000000 & 3.349207000000 & -0.107201000000 & $\mathrm{H}$ & 1.420640000000 & -0.408630000000 & 1.803596000000 \\
\hline $\mathrm{H}$ & -1.611073000000 & 2.034989000000 & -0.115804000000 & $\mathrm{H}$ & 0.922622000000 & 1.127823000000 & 0.965711000000 \\
\hline 0 & -0.691187000000 & -2.250158000000 & -0.012797000000 & $\mathrm{O}$ & -1.972832000000 & 1.270775000000 & -1.959510000000 \\
\hline $\mathrm{N}$ & 1.769963000000 & 1.681120000000 & -0.006591000000 & $\mathrm{~N}$ & -0.891612000000 & -1.709050000000 & 1.309053000000 \\
\hline $\mathrm{C}$ & 1.729684000000 & -0.600450000000 & -0.002494000000 & $\mathrm{C}$ & -2.466437000000 & -1.064667000000 & -0.201908000000 \\
\hline C & 2.317394000000 & -1.864894000000 & 0.010433000000 & C & -3.640859000000 & -1.219439000000 & -0.948476000000 \\
\hline C & 2.566084000000 & 0.543677000000 & 0.003008000000 & $\mathrm{C}$ & -2.058422000000 & -2.108180000000 & 0.665802000000 \\
\hline $\mathrm{C}$ & 3.953495000000 & 0.436388000000 & 0.018517000000 & $\mathrm{C}$ & -2.788079000000 & -3.288458000000 & 0.792584000000 \\
\hline $\mathrm{C}$ & 3.715087000000 & -1.982581000000 & 0.027421000000 & C & -4.373874000000 & -2.400435000000 & -0.826076000000 \\
\hline C & 4.520442000000 & -0.845727000000 & 0.031132000000 & $\mathrm{C}$ & -3.951467000000 & -3.423243000000 & 0.032054000000 \\
\hline $\mathrm{H}$ & 1.688675000000 & -2.743444000000 & 0.005858000000 & $\mathrm{H}$ & -3.970678000000 & -0.430763000000 & -1.613627000000 \\
\hline $\mathrm{H}$ & 4.171528000000 & -2.966550000000 & 0.036381000000 & $\mathrm{H}$ & -5.281399000000 & -2.529133000000 & -1.405325000000 \\
\hline $\mathrm{H}$ & 5.600407000000 & -0.946325000000 & 0.042781000000 & $\mathrm{H}$ & -4.531888000000 & -4.335804000000 & 0.107947000000 \\
\hline $\mathrm{H}$ & 4.591893000000 & 1.311741000000 & 0.020056000000 & $\mathrm{H}$ & -2.470178000000 & -4.086446000000 & 1.452780000000 \\
\hline $\mathrm{Li}$ & -1.350774000000 & -3.838600000000 & -0.006860000000 & C & 1.677354000000 & -0.390974000000 & -0.843501000000 \\
\hline $\mathrm{C}$ & 2.247618000000 & 3.042297000000 & -0.028825000000 & $\mathrm{~N}$ & 0.959439000000 & -0.349039000000 & -1.793988000000 \\
\hline $\mathrm{H}$ & 1.842574000000 & 3.610981000000 & 0.815365000000 & $\mathrm{Li}$ & -0.789967000000 & -0.102019000000 & -2.566330000000 \\
\hline $\mathrm{H}$ & 3.333475000000 & 3.052108000000 & 0.042142000000 & $\mathrm{C}$ & -0.095918000000 & -2.557404000000 & 2.174860000000 \\
\hline $\mathrm{H}$ & 1.955517000000 & 3.550503000000 & -0.955528000000 & $\mathrm{H}$ & 0.340492000000 & -1.963207000000 & 2.978740000000 \\
\hline $\mathrm{C}$ & -2.174536000000 & -0.424571000000 & 0.002965000000 & $\mathrm{H}$ & -0.735116000000 & -3.316336000000 & 2.624415000000 \\
\hline $\mathrm{C}$ & -2.817495000000 & -0.150029000000 & 1.215469000000 & $\mathrm{H}$ & 0.711021000000 & -3.055627000000 & 1.626667000000 \\
\hline $\mathrm{C}$ & -2.896554000000 & -0.295172000000 & -1.189222000000 & C & 3.080138000000 & -0.682620000000 & -0.510686000000 \\
\hline C & -4.154643000000 & 0.245466000000 & 1.236117000000 & C & 3.685933000000 & -1.807022000000 & -1.090547000000 \\
\hline $\mathrm{C}$ & -4.232886000000 & 0.103453000000 & -1.170237000000 & $\mathrm{C}$ & 3.832624000000 & 0.139609000000 & 0.336008000000 \\
\hline $\mathrm{C}$ & -4.866244000000 & 0.373885000000 & 0.043064000000 & C & 5.017892000000 & -2.106462000000 & -0.816077000000 \\
\hline $\mathrm{H}$ & -2.261821000000 & -0.233927000000 & 2.143298000000 & $\mathrm{C}$ & 5.170831000000 & -0.150931000000 & 0.588214000000 \\
\hline $\mathrm{H}$ & -2.403047000000 & -0.492521000000 & -2.134986000000 & $\mathrm{C}$ & 5.765507000000 & -1.278106000000 & 0.020625000000 \\
\hline $\mathrm{H}$ & -4.638497000000 & 0.460829000000 & 2.182781000000 & $\mathrm{H}$ & 3.105937000000 & -2.438531000000 & -1.752828000000 \\
\hline $\mathrm{H}$ & -4.777234000000 & 0.209826000000 & -2.102495000000 & $\mathrm{H}$ & 3.373849000000 & 1.010672000000 & 0.786515000000 \\
\hline $\mathrm{H}$ & -5.904006000000 & 0.688054000000 & 0.058214000000 & $\mathrm{H}$ & 5.473805000000 & -2.983418000000 & -1.262113000000 \\
\hline & & & & $\mathrm{H}$ & 5.749021000000 & 0.501800000000 & 1.232536000000 \\
\hline & & & & $\mathrm{H}$ & 6.804296000000 & -1.508323000000 & 0.228428000000 \\
\hline & & & & C & -1.094042000000 & 2.541014000000 & -0.164854000000 \\
\hline & & & & $\mathrm{C}$ & -1.261630000000 & 2.805864000000 & 1.201055000000 \\
\hline & & & & C & -0.579966000000 & 3.546560000000 & -0.995044000000 \\
\hline & & & & $\mathrm{C}$ & -0.909903000000 & 4.046011000000 & 1.727217000000 \\
\hline & & & & C & -0.213193000000 & 4.780056000000 & -0.466307000000 \\
\hline & & & & $\mathrm{C}$ & -0.378449000000 & 5.032916000000 & 0.896492000000 \\
\hline & & & & $\mathrm{H}$ & -1.681306000000 & 2.041464000000 & 1.843478000000 \\
\hline & & & & $\mathrm{H}$ & -0.471154000000 & 3.347144000000 & -2.053931000000 \\
\hline & & & & $\mathrm{H}$ & -1.055475000000 & 4.245028000000 & 2.783045000000 \\
\hline & & & & $\mathrm{H}$ & 0.195813000000 & 5.546583000000 & -1.115001000000 \\
\hline & & & & $\mathrm{H}$ & -0.101342000000 & 5.997424000000 & 1.307462000000 \\
\hline
\end{tabular}




\begin{tabular}{|c|c|c|c|c|c|c|c|}
\hline \multicolumn{4}{|c|}{$\begin{array}{c}\text { Intermediate } 15^{`} \text { of exo-1 path } \\
\mathbf{E}_{\text {tot. }}= \\
-1118.58780 \text { a.u., } v_{\text {im. }}=0(-1118.27609)\end{array}$} & \multicolumn{4}{|c|}{$\begin{array}{c}\text { TS2 of exo-1 path } \\
E_{\text {tot. }}=-1118.58583 \text { a.u., } v_{\text {im. }}=-75.8 \mathrm{~cm}^{-1}(-1118.27147)\end{array}$} \\
\hline $\mathrm{C}$ & 0.700639000000 & -0.573580000000 & -0.721447000000 & C & 0.748766000000 & -0.805020000000 & -0.644518000000 \\
\hline $\mathrm{C}$ & 1.550415000000 & 0.184138000000 & 0.089530000000 & C & 1.412296000000 & 0.044684000000 & 0.219299000000 \\
\hline C & -0.755260000000 & -0.412368000000 & -0.888075000000 & C & -0.698643000000 & -0.773620000000 & -0.976842000000 \\
\hline $\mathrm{C}$ & 1.203706000000 & 1.395216000000 & 0.818627000000 & $\mathrm{C}$ & 0.822834000000 & 1.216319000000 & 0.894172000000 \\
\hline $\mathrm{H}$ & -1.146835000000 & -1.113078000000 & -1.624386000000 & $\mathrm{H}$ & -1.020489000000 & -1.724212000000 & -1.402501000000 \\
\hline $\mathrm{H}$ & -1.000553000000 & 0.594256000000 & -1.233678000000 & $\mathrm{H}$ & -0.917092000000 & -0.002177000000 & -1.725933000000 \\
\hline 0 & 1.552594000000 & 1.535743000000 & 2.016556000000 & 0 & 1.165587000000 & 1.487633000000 & 2.093519000000 \\
\hline $\mathrm{N}$ & 1.364005000000 & -1.726595000000 & -1.098548000000 & $\mathrm{~N}$ & 1.606705000000 & -1.822684000000 & -1.040989000000 \\
\hline $\mathrm{C}$ & 2.779498000000 & -0.561633000000 & 0.239658000000 & $\mathrm{C}$ & 2.741447000000 & -0.481074000000 & 0.398055000000 \\
\hline C & 3.987130000000 & -0.328016000000 & 0.912711000000 & C & 3.863006000000 & -0.076367000000 & 1.138463000000 \\
\hline C & 2.634766000000 & -1.737229000000 & -0.537389000000 & C & 2.829126000000 & -1.643799000000 & -0.412971000000 \\
\hline C & 3.664731000000 & -2.673966000000 & -0.657771000000 & C & 4.002484000000 & -2.400513000000 & -0.494691000000 \\
\hline C & 5.010925000000 & -1.261253000000 & 0.800706000000 & C & 5.028596000000 & -0.827152000000 & 1.058298000000 \\
\hline $\mathrm{C}$ & 4.851155000000 & -2.421647000000 & 0.023104000000 & $\mathrm{C}$ & 5.097792000000 & -1.977302000000 & 0.249778000000 \\
\hline $\mathrm{H}$ & 4.112220000000 & 0.561843000000 & 1.517743000000 & $\mathrm{H}$ & 3.806644000000 & 0.802792000000 & 1.768378000000 \\
\hline $\mathrm{H}$ & 5.947004000000 & -1.095421000000 & 1.321952000000 & $\mathrm{H}$ & 5.900500000000 & -0.526786000000 & 1.628796000000 \\
\hline $\mathrm{H}$ & 5.664062000000 & -3.135599000000 & -0.045288000000 & $\mathrm{H}$ & 6.019266000000 & -2.547310000000 & 0.209185000000 \\
\hline $\mathrm{H}$ & 3.552716000000 & -3.574106000000 & -1.249975000000 & $\mathrm{H}$ & 4.065407000000 & -3.291958000000 & -1.107636000000 \\
\hline C & -1.548850000000 & -0.593899000000 & 0.518252000000 & C & -1.570183000000 & -0.434992000000 & 0.281600000000 \\
\hline $\mathrm{N}$ & -1.048230000000 & -0.336577000000 & 1.624536000000 & $\mathrm{~N}$ & -1.148460000000 & 0.273201000000 & 1.224273000000 \\
\hline $\mathrm{Li}$ & 0.374440000000 & 0.159742000000 & 2.714176000000 & $\mathrm{Li}$ & -0.329026000000 & 0.727256000000 & 2.864448000000 \\
\hline C & 0.803248000000 & -2.817069000000 & -1.881693000000 & C & 1.277220000000 & -2.946379000000 & -1.900906000000 \\
\hline $\mathrm{H}$ & 0.188764000000 & -2.423293000000 & -2.691008000000 & $\mathrm{H}$ & 0.566011000000 & -2.638272000000 & -2.666982000000 \\
\hline $\mathrm{H}$ & 1.615142000000 & -3.390720000000 & -2.325954000000 & $\mathrm{H}$ & 2.180583000000 & -3.290364000000 & -2.404358000000 \\
\hline $\mathrm{H}$ & 0.195828000000 & -3.485120000000 & -1.264281000000 & $\mathrm{H}$ & 0.851629000000 & -3.782133000000 & -1.335733000000 \\
\hline C & -2.976231000000 & -1.062342000000 & 0.317483000000 & C & -2.965076000000 & -1.014130000000 & 0.256299000000 \\
\hline $\mathrm{C}$ & -3.617645000000 & -1.731315000000 & 1.370305000000 & $\mathrm{C}$ & -3.622308000000 & -1.293789000000 & 1.462235000000 \\
\hline C & -3.706309000000 & -0.824954000000 & -0.855089000000 & C & -3.649447000000 & -1.254343000000 & -0.942906000000 \\
\hline C & -4.935588000000 & -2.159212000000 & 1.253112000000 & C & -4.915214000000 & -1.806266000000 & 1.471953000000 \\
\hline $\mathrm{C}$ & -5.036344000000 & -1.234553000000 & -0.968366000000 & C & -4.953905000000 & -1.749158000000 & -0.935937000000 \\
\hline $\mathrm{C}$ & -5.654475000000 & -1.909243000000 & 0.081583000000 & $\mathrm{C}$ & -5.589740000000 & -2.032938000000 & 0.270506000000 \\
\hline $\mathrm{H}$ & -3.056652000000 & -1.898821000000 & 2.282181000000 & $\mathrm{H}$ & -3.100741000000 & -1.103256000000 & 2.392546000000 \\
\hline $\mathrm{H}$ & -3.252075000000 & -0.297056000000 & -1.685316000000 & $\mathrm{H}$ & -3.177558000000 & -1.036730000000 & -1.893995000000 \\
\hline $\mathrm{H}$ & -5.407357000000 & -2.685499000000 & 2.076364000000 & $\mathrm{H}$ & -5.400437000000 & -2.028364000000 & 2.416316000000 \\
\hline $\mathrm{H}$ & -5.587754000000 & -1.024500000000 & -1.878727000000 & $\mathrm{H}$ & -5.471560000000 & -1.912542000000 & -1.874871000000 \\
\hline $\mathrm{H}$ & -6.684737000000 & -2.235420000000 & -0.008900000000 & $\mathrm{H}$ & -6.600129000000 & -2.426173000000 & 0.276488000000 \\
\hline C & 0.513974000000 & 2.528642000000 & 0.134483000000 & C & 0.281685000000 & 2.351247000000 & 0.056724000000 \\
\hline $\mathrm{C}$ & 0.649800000000 & 2.740214000000 & -1.244421000000 & C & 0.461840000000 & 2.414493000000 & -1.330031000000 \\
\hline $\mathrm{C}$ & -0.221269000000 & 3.447836000000 & 0.896371000000 & C & -0.356644000000 & 3.423862000000 & 0.695433000000 \\
\hline $\mathrm{C}$ & 0.053495000000 & 3.843364000000 & -1.850488000000 & $\mathrm{C}$ & -0.007923000000 & 3.505105000000 & -2.062179000000 \\
\hline $\mathrm{C}$ & -0.829621000000 & 4.539533000000 & 0.288305000000 & C & -0.834125000000 & 4.507353000000 & -0.032873000000 \\
\hline $\mathrm{C}$ & -0.692580000000 & 4.740307000000 & -1.086985000000 & C & -0.664855000000 & 4.551225000000 & -1.418567000000 \\
\hline $\mathrm{H}$ & 1.239050000000 & 2.048727000000 & -1.834363000000 & $\mathrm{H}$ & 0.994201000000 & 1.618574000000 & -1.836201000000 \\
\hline $\mathrm{H}$ & -0.313264000000 & 3.291149000000 & 1.963714000000 & $\mathrm{H}$ & -0.462571000000 & 3.404359000000 & 1.772787000000 \\
\hline $\mathrm{H}$ & 0.175134000000 & 4.005554000000 & -2.915493000000 & $\mathrm{H}$ & 0.150354000000 & 3.540565000000 & -3.134641000000 \\
\hline $\mathrm{H}$ & -1.409164000000 & 5.236350000000 & 0.883072000000 & $\mathrm{H}$ & -1.332160000000 & 5.324001000000 & 0.478453000000 \\
\hline $\mathrm{H}$ & -1.161889000000 & 5.595964000000 & -1.559588000000 & $\mathrm{H}$ & -1.031366000000 & 5.398872000000 & -1.986938000000 \\
\hline
\end{tabular}




\begin{tabular}{|c|c|c|c|c|c|c|c|}
\hline \multicolumn{4}{|c|}{$\begin{array}{c}\text { Product of exo-1 path } \\
E_{\text {tot. }}=-1118.59868 \text { a.u., } v_{\text {im. }}=0(-1118.28279)\end{array}$} & \multicolumn{4}{|c|}{$\begin{array}{c}\text { PhCN } \\
E_{\text {tot. }}=-324.58164 \text { a.u., } v_{\text {im. }}=0(-324.51303)\end{array}$} \\
\hline C & -0.449912000000 & -1.331389000000 & 0.423744000000 & $\mathrm{~N}$ & 3.196661000000 & 0.000012000000 & -0.000027000000 \\
\hline C & -1.040827000000 & -0.380998000000 & -0.358597000000 & C & 2.040619000000 & -0.000025000000 & -0.000039000000 \\
\hline C & 0.992120000000 & -1.340504000000 & 0.786908000000 & C & 0.609279000000 & -0.000020000000 & -0.000014000000 \\
\hline C & -0.302385000000 & 0.831669000000 & -0.876624000000 & C & -0.089588000000 & -1.216516000000 & -0.000002000000 \\
\hline $\mathrm{H}$ & 1.446805000000 & -2.324654000000 & 0.628616000000 & C & -0.089563000000 & 1.216504000000 & -0.000002000000 \\
\hline $\mathrm{H}$ & 1.153459000000 & -1.109473000000 & 1.849901000000 & C & -1.480189000000 & -1.209417000000 & 0.000021000000 \\
\hline 0 & -0.501514000000 & 1.088203000000 & -2.195582000000 & C & -1.480154000000 & 1.209438000000 & 0.000021000000 \\
\hline $\mathrm{N}$ & -1.377880000000 & -2.294401000000 & 0.807034000000 & C & -2.175532000000 & 0.000015000000 & 0.000033000000 \\
\hline C & -2.420181000000 & -0.757164000000 & -0.506401000000 & $\mathrm{H}$ & 0.457861000000 & -2.150779000000 & -0.000011000000 \\
\hline C & -3.519171000000 & -0.210430000000 & -1.184498000000 & $\mathrm{H}$ & 0.457923000000 & 2.150745000000 & -0.000011000000 \\
\hline C & -2.592230000000 & -1.962869000000 & 0.230394000000 & $\mathrm{H}$ & -2.021156000000 & -2.148190000000 & 0.000029000000 \\
\hline C & -3.822219000000 & -2.624278000000 & 0.291676000000 & $\mathrm{H}$ & -2.0211111000000 & 2.148218000000 & 0.000030000000 \\
\hline C & -4.744625000000 & -0.861736000000 & -1.117962000000 & $\mathrm{H}$ & -3.259383000000 & 0.000036000000 & 0.000050000000 \\
\hline C & -4.894490000000 & -2.056235000000 & -0.388522000000 & & & & \\
\hline $\mathrm{H}$ & -3.397758000000 & 0.697738000000 & -1.761369000000 & & & & \\
\hline $\mathrm{H}$ & -5.600699000000 & -0.449729000000 & -1.640937000000 & & & & \\
\hline $\mathrm{H}$ & -5.861548000000 & -2.546165000000 & -0.359176000000 & & & & \\
\hline $\mathrm{H}$ & -3.944297000000 & -3.549085000000 & 0.844008000000 & & & & \\
\hline C & 1.750355000000 & -0.325930000000 & -0.064574000000 & & & & \\
\hline $\mathrm{N}$ & 1.214182000000 & 0.614249000000 & -0.737347000000 & & & & \\
\hline $\mathrm{Li}$ & 1.193536000000 & 1.573320000000 & -2.550456000000 & & & & \\
\hline C & -1.115156000000 & -3.514134000000 & 1.546624000000 & & & & \\
\hline $\mathrm{H}$ & -0.179990000000 & -3.417340000000 & 2.097040000000 & & & & \\
\hline $\mathrm{H}$ & -1.913601000000 & -3.690831000000 & 2.270501000000 & & & & \\
\hline $\mathrm{H}$ & -1.043744000000 & -4.384148000000 & 0.884404000000 & & & & \\
\hline C & 3.235326000000 & -0.496137000000 & -0.118618000000 & & & & \\
\hline C & 3.913136000000 & -0.424665000000 & -1.342114000000 & & & & \\
\hline C & 3.977279000000 & -0.732576000000 & 1.046410000000 & & & & \\
\hline C & 5.296168000000 & -0.573196000000 & -1.400850000000 & & & & \\
\hline C & 5.363468000000 & -0.864572000000 & 0.990789000000 & & & & \\
\hline C & 6.027463000000 & -0.786491000000 & -0.232353000000 & & & & \\
\hline $\mathrm{H}$ & 3.344566000000 & -0.284252000000 & -2.254219000000 & & & & \\
\hline $\mathrm{H}$ & 3.477536000000 & -0.791879000000 & 2.006539000000 & & & & \\
\hline $\mathrm{H}$ & 5.802986000000 & -0.530058000000 & -2.358466000000 & & & & \\
\hline $\mathrm{H}$ & 5.924365000000 & -1.028427000000 & 1.904028000000 & & & & \\
\hline $\mathrm{H}$ & 7.104891000000 & -0.897381000000 & -0.275908000000 & & & & \\
\hline C & -0.620929000000 & 2.065777000000 & 0.025227000000 & & & & \\
\hline C & -0.381303000000 & 2.072437000000 & 1.404653000000 & & & & \\
\hline C & -1.177484000000 & 3.205158000000 & -0.556930000000 & & & & \\
\hline C & -0.677676000000 & 3.192158000000 & 2.177965000000 & & & & \\
\hline c & -1.484601000000 & 4.328314000000 & 0.214694000000 & & & & \\
\hline C & -1.232167000000 & 4.328171000000 & 1.584371000000 & & & & \\
\hline $\mathrm{H}$ & 0.033913000000 & 1.194471000000 & 1.886980000000 & & & & \\
\hline $\mathrm{H}$ & -1.374270000000 & 3.185231000000 & -1.621754000000 & & & & \\
\hline $\mathrm{H}$ & -0.481298000000 & 3.178915000000 & 3.244991000000 & & & & \\
\hline $\mathrm{H}$ & -1.924936000000 & 5.201501000000 & -0.255785000000 & & & & \\
\hline $\mathrm{H}$ & -1.468755000000 & 5.198773000000 & 2.186346000000 & & & & \\
\hline
\end{tabular}


Table S12. Optimized geometries of stationary points of interaction of lithium enolate $\mathbf{1 5}$ and benzonitrile. $\mathrm{PCM} / \mathrm{MP} 2 / / \mathrm{HF} / 6-311++\mathrm{G}(\mathrm{d}, \mathrm{p})$ calculations (Benzene, $\left.25^{\circ} \mathrm{C}\right)$. Coordinates are in angstroms. Sum of electronic and thermal Free Energies in a.u. are in parentheses.

\begin{tabular}{|c|c|c|c|c|c|c|c|}
\hline \multicolumn{4}{|c|}{$\begin{array}{c}15 \\
E_{\text {tot. }}=-791.63053 \text { a.u., } v_{\text {im. }}=0(-791.39286)\end{array}$} & \multicolumn{4}{|c|}{$\begin{array}{c}\text { Product of exo-1 path } \\
E_{\text {tot. }}=-1118.30222 \text { a.u., } v_{\text {im. }}=0(-1114.95989)\end{array}$} \\
\hline C & 0.405578000000 & 1.351577000000 & -0.071168000000 & $\mathrm{C}$ & 0.464107000000 & -1.352029000000 & -0.395401000000 \\
\hline C & 0.331701000000 & -0.131890000000 & -0.029069000000 & $\mathrm{C}$ & 1.032687000000 & -0.393702000000 & 0.352002000000 \\
\hline C & -0.543858000000 & 2.291846000000 & -0.198163000000 & C & -0.984947000000 & -1.390817000000 & -0.733217000000 \\
\hline C & -0.760572000000 & -0.942114000000 & -0.011930000000 & $\mathrm{C}$ & 0.278732000000 & 0.803113000000 & 0.881598000000 \\
\hline $\mathrm{H}$ & -0.290348000000 & 3.333876000000 & -0.229022000000 & $\mathrm{H}$ & -1.420787000000 & -2.362418000000 & -0.519213000000 \\
\hline $\mathrm{H}$ & -1.578457000000 & 2.038340000000 & -0.273842000000 & $\mathrm{H}$ & -1.159050000000 & -1.207291000000 & -1.792200000000 \\
\hline $\mathrm{O}$ & -0.687301000000 & -2.220832000000 & -0.005153000000 & $\mathrm{O}$ & 0.498737000000 & 1.044629000000 & 2.184360000000 \\
\hline $\mathrm{N}$ & 1.762186000000 & 1.669127000000 & 0.024470000000 & $\mathrm{~N}$ & 1.396682000000 & -2.287879000000 & -0.801256000000 \\
\hline C & 1.723517000000 & -0.603720000000 & -0.003569000000 & $\mathrm{C}$ & 2.430381000000 & -0.736059000000 & 0.468760000000 \\
\hline C & 2.303565000000 & -1.856497000000 & 0.005732000000 & $\mathrm{C}$ & 3.523339000000 & -0.159327000000 & 1.116231000000 \\
\hline C & 2.551073000000 & 0.531349000000 & 0.019198000000 & $\mathrm{C}$ & 2.605125000000 & -1.925493000000 & -0.254293000000 \\
\hline C & 3.926376000000 & 0.425648000000 & 0.040734000000 & $\mathrm{C}$ & 3.843990000000 & -2.559707000000 & -0.335502000000 \\
\hline C & 3.696418000000 & -1.976907000000 & 0.031862000000 & $\mathrm{C}$ & 4.753020000000 & -0.776822000000 & 1.031112000000 \\
\hline C & 4.492659000000 & -0.850906000000 & 0.047574000000 & $\mathrm{C}$ & 4.908730000000 & -1.969188000000 & 0.311870000000 \\
\hline $\mathrm{H}$ & 1.686432000000 & -2.731062000000 & -0.010272000000 & $\mathrm{H}$ & 3.396870000000 & 0.744869000000 & 1.681277000000 \\
\hline $\mathrm{H}$ & 4.146715000000 & -2.954167000000 & 0.036391000000 & $\mathrm{H}$ & 5.604349000000 & -0.345427000000 & 1.527287000000 \\
\hline $\mathrm{H}$ & 5.564189000000 & -0.948775000000 & 0.063736000000 & $\mathrm{H}$ & 5.877343000000 & -2.435541000000 & 0.266144000000 \\
\hline $\mathrm{H}$ & 4.561879000000 & 1.291148000000 & 0.050782000000 & $\mathrm{H}$ & 3.974525000000 & -3.478166000000 & -0.878651000000 \\
\hline $\mathrm{Li}$ & -1.365063000000 & -3.799120000000 & 0.018824000000 & $\mathrm{C}$ & -1.733536000000 & -0.354272000000 & 0.090546000000 \\
\hline C & 2.255444000000 & 3.016674000000 & -0.053873000000 & $\mathrm{~N}$ & -1.204677000000 & 0.567221000000 & 0.752918000000 \\
\hline $\mathrm{H}$ & 1.767557000000 & 3.642086000000 & 0.685872000000 & $\mathrm{Li}$ & -1.174640000000 & 1.418057000000 & 2.657138000000 \\
\hline $\mathrm{H}$ & 3.315873000000 & 3.030068000000 & 0.148047000000 & $\mathrm{C}$ & 1.136865000000 & -3.536413000000 & -1.476627000000 \\
\hline $\mathrm{H}$ & 2.084604000000 & 3.449122000000 & -1.037846000000 & $\mathrm{H}$ & 0.195067000000 & -3.478160000000 & -2.004288000000 \\
\hline C & -2.172658000000 & -0.420623000000 & 0.011603000000 & $\mathrm{H}$ & 1.913012000000 & -3.730266000000 & -2.206918000000 \\
\hline C & -2.778277000000 & -0.076532000000 & 1.212938000000 & $\mathrm{H}$ & 1.095209000000 & -4.371031000000 & -0.781049000000 \\
\hline C & -2.917986000000 & -0.368228000000 & -1.159988000000 & $\mathrm{C}$ & -3.227482000000 & -0.501417000000 & 0.118107000000 \\
\hline C & -4.106977000000 & 0.318540000000 & 1.242556000000 & $\mathrm{C}$ & -3.908297000000 & -0.536509000000 & 1.329325000000 \\
\hline C & -4.244958000000 & 0.032031000000 & -1.132476000000 & $\mathrm{C}$ & -3.953520000000 & -0.610846000000 & -1.062334000000 \\
\hline C & -4.843932000000 & 0.375783000000 & 0.069877000000 & $\mathrm{C}$ & -5.286334000000 & -0.669997000000 & 1.361572000000 \\
\hline $\mathrm{H}$ & -2.205562000000 & -0.102747000000 & 2.123154000000 & $\mathrm{C}$ & -5.334170000000 & -0.729044000000 & -1.032052000000 \\
\hline $\mathrm{H}$ & -2.454298000000 & -0.623202000000 & -2.096987000000 & $\mathrm{C}$ & -6.004436000000 & -0.761382000000 & 0.179568000000 \\
\hline $\mathrm{H}$ & -4.562881000000 & 0.588495000000 & 2.178895000000 & $\mathrm{H}$ & -3.357144000000 & -0.480461000000 & 2.250544000000 \\
\hline $\mathrm{H}$ & -4.807618000000 & 0.081093000000 & -2.048219000000 & $\mathrm{H}$ & -3.450804000000 & -0.584685000000 & -2.012468000000 \\
\hline $\mathrm{H}$ & -5.872471000000 & 0.689671000000 & 0.091741000000 & $\mathrm{H}$ & -5.797515000000 & -0.706362000000 & 2.307105000000 \\
\hline & & & & $\mathrm{H}$ & -5.883171000000 & -0.795760000000 & -1.954275000000 \\
\hline & & & & $\mathrm{H}$ & -7.074805000000 & -0.860663000000 & 0.203197000000 \\
\hline & & & & $\mathrm{C}$ & 0.587157000000 & 2.047587000000 & 0.003459000000 \\
\hline & & & & $\mathrm{C}$ & 0.295115000000 & 2.090106000000 & -1.358503000000 \\
\hline & & & & $\mathrm{C}$ & 1.189917000000 & 3.156434000000 & 0.576827000000 \\
\hline & & & & $\mathrm{C}$ & 0.585929000000 & 3.208028000000 & -2.118510000000 \\
\hline & & & & $\mathrm{C}$ & 1.489911000000 & 4.281314000000 & -0.183892000000 \\
\hline & & & & $\mathrm{C}$ & 1.187406000000 & 4.314124000000 & -1.532161000000 \\
\hline & & & & $\mathrm{H}$ & -0.159913000000 & 1.242711000000 & -1.838689000000 \\
\hline & & & & $\mathrm{H}$ & 1.428926000000 & 3.127014000000 & 1.621669000000 \\
\hline & & & & $\mathrm{H}$ & 0.348402000000 & 3.217428000000 & -3.168164000000 \\
\hline & & & & $\mathrm{H}$ & 1.962955000000 & 5.128268000000 & 0.282530000000 \\
\hline & & & & $\mathrm{H}$ & 1.418382000000 & 5.183264000000 & -2.122676000000 \\
\hline
\end{tabular}




\section{PhCN}

$E_{\text {tot. }}=-323.62037$ a.u., $v_{\text {im. }}=0(-324.54478)$

$\begin{array}{llll}N & 3.173401000000 & 0.000202000000 & -0.000168000000\end{array}$

C $2.041803000000 \quad-0.000378000000 \quad 0.000048000000$

C $\quad 0.598415000000 \quad-0.000173000000 \quad 0.000028000000$

C $-0.089363000000 \quad-1.207982000000 \quad 0.000023000000$

C $-0.089041000000 \quad 1.207821000000 \quad 0.000023000000$

C $-1.472431000000 \quad-1.203001000000 \quad 0.000020000000$

C $-1.472109000000 \quad 1.203214000000 \quad 0.000020000000$

C $-2.161781000000 \quad 0.000198000000 \quad 0.000017000000$

H $\quad 0.453748000000 \quad-2.134824000000 \quad 0.000026000000$

H $\quad 0.454320000000 \quad 2.134517000000 \quad 0.000027000000$

$\begin{array}{llll}\text { H } & -2.009241000000 & -2.133852000000 & 0.000018000000\end{array}$

$\begin{array}{llll}H & -2.008671000000 & 2.134208000000 & 0.000018000000\end{array}$

$\begin{array}{llll}\text { H } & -3.236928000000 & 0.000343000000 & 0.000013000000\end{array}$ 
Table S13. Optimized geometries of stationary points of interaction of lithium enolate 24 and HCN. PCM/B3LYP/6$311++\mathrm{G}(\mathrm{d}, \mathrm{p})$ calculations $\left(\mathrm{THF},-78^{\circ} \mathrm{C}\right)$. Coordinates are in angstroms. Sum of electronic and thermal Free Energies in a.u. are in parentheses.

\begin{tabular}{|c|c|c|c|c|c|c|c|}
\hline \multicolumn{4}{|c|}{$\begin{array}{c}24 \\
E_{\text {tot. }}=-391.96681 \text { a.u., } v_{\text {im. }}=0(-391.86111)\end{array}$} & \multicolumn{4}{|c|}{$\begin{array}{c}\text { TS1 of exo-1 path } \\
E_{\text {tot. }}=-485.42164 \text { a.u., } v_{\text {im. }}=-271.2 \mathrm{~cm}^{-1}(-485.29592)\end{array}$} \\
\hline C & -0.830206000000 & 0.990315000000 & 0.015961000000 & $\mathrm{C}$ & -0.526110000000 & 0.836460000000 & -0.593638000000 \\
\hline $\mathrm{C}$ & 0.371552000000 & 0.126253000000 & 0.022861000000 & $\mathrm{C}$ & -0.148023000000 & -0.558860000000 & -0.449785000000 \\
\hline $\mathrm{C}$ & -0.839022000000 & 2.346372000000 & 0.181912000000 & $\mathrm{C}$ & 0.351820000000 & 1.850620000000 & -0.981308000000 \\
\hline $\mathrm{C}$ & 1.654102000000 & 0.654215000000 & -0.147192000000 & $\mathrm{C}$ & 1.134490000000 & -1.015668000000 & -0.863106000000 \\
\hline $\mathrm{H}$ & -1.769011000000 & 2.898808000000 & 0.105821000000 & $\mathrm{H}$ & -0.032303000000 & 2.862183000000 & -1.069708000000 \\
\hline $\mathrm{H}$ & 0.054254000000 & 2.920055000000 & 0.394311000000 & $\mathrm{H}$ & 1.257421000000 & 1.647511000000 & -1.537535000000 \\
\hline $\mathrm{H}$ & 1.718554000000 & 1.728057000000 & -0.373046000000 & $\mathrm{H}$ & 1.669509000000 & -0.387028000000 & -1.589887000000 \\
\hline $\mathrm{O}$ & 2.759833000000 & 0.016559000000 & -0.090763000000 & 0 & 1.704777000000 & -2.079086000000 & -0.480495000000 \\
\hline $\mathrm{C}$ & -2.098215000000 & 0.281262000000 & -0.146773000000 & $\mathrm{C}$ & -1.852772000000 & 1.178810000000 & -0.141383000000 \\
\hline C & 0.202235000000 & -1.296133000000 & 0.136517000000 & C & -1.045320000000 & -1.476515000000 & 0.172648000000 \\
\hline C & -1.021896000000 & -1.893214000000 & 0.093510000000 & $\mathrm{C}$ & -2.293537000000 & -1.097234000000 & 0.590880000000 \\
\hline $\mathrm{H}$ & -2.994949000000 & 0.882529000000 & -0.265071000000 & $\mathrm{H}$ & -2.174406000000 & 2.209812000000 & -0.252131000000 \\
\hline C & -2.191639000000 & -1.071896000000 & -0.090981000000 & $\mathrm{H}$ & 1.781779000000 & 2.822190000000 & 1.002445000000 \\
\hline $\mathrm{H}$ & 1.101110000000 & -1.898003000000 & 0.217632000000 & C & 1.808842000000 & 1.759210000000 & 0.849988000000 \\
\hline $\mathrm{H}$ & -1.122542000000 & -2.970648000000 & 0.159461000000 & $\mathrm{~N}$ & 2.202057000000 & 0.686937000000 & 1.110767000000 \\
\hline $\mathrm{H}$ & -3.163463000000 & -1.547897000000 & -0.181530000000 & C & -2.695401000000 & 0.260359000000 & 0.420385000000 \\
\hline \multirow[t]{4}{*}{$\mathrm{Li}$} & 4.205305000000 & -0.989472000000 & 0.091212000000 & $\mathrm{H}$ & -0.719770000000 & -2.506083000000 & 0.274392000000 \\
\hline & & & & $\mathrm{H}$ & -2.976693000000 & -1.816048000000 & 1.028379000000 \\
\hline & & & & $\mathrm{H}$ & -3.686508000000 & 0.567091000000 & 0.739782000000 \\
\hline & & & & $\mathrm{Li}$ & 2.474806000000 & -1.332863000000 & 1.148256000000 \\
\hline \multicolumn{4}{|c|}{ Product of exo-1 path } & & & & \\
\hline \multicolumn{4}{|c|}{$E_{\text {tot. }}=-485.47741$ a.u., $v_{\text {im. }}=0(-485.34413)$} & & & & \\
\hline $\mathrm{C}$ & 0.627136000000 & 0.887696000000 & 0.152325000000 & & & & \\
\hline $\mathrm{C}$ & 0.146415000000 & -0.426677000000 & 0.207081000000 & & & & \\
\hline $\mathrm{C}$ & -0.377986000000 & 2.008030000000 & 0.291745000000 & & & & \\
\hline $\mathrm{C}$ & -1.344712000000 & -0.653262000000 & 0.463592000000 & & & & \\
\hline $\mathrm{H}$ & -0.018557000000 & 2.933876000000 & -0.162533000000 & & & & \\
\hline $\mathrm{H}$ & -0.554946000000 & 2.231710000000 & 1.354958000000 & & & & \\
\hline $\mathrm{H}$ & -1.511928000000 & -0.402405000000 & 1.538143000000 & & & & \\
\hline 0 & -1.827767000000 & -1.872236000000 & 0.127427000000 & & & & \\
\hline $\mathrm{C}$ & 1.985997000000 & 1.123163000000 & -0.069331000000 & & & & \\
\hline C & 1.029558000000 & -1.493432000000 & 0.042192000000 & & & & \\
\hline $\mathrm{C}$ & 2.389250000000 & -1.256774000000 & -0.161566000000 & & & & \\
\hline $\mathrm{H}$ & 2.353987000000 & 2.142718000000 & -0.131408000000 & & & & \\
\hline $\mathrm{H}$ & -2.283618000000 & 2.357620000000 & -0.831502000000 & & & & \\
\hline C & -1.697026000000 & 1.594321000000 & -0.315870000000 & & & & \\
\hline $\mathrm{N}$ & -2.149857000000 & 0.409808000000 & -0.268271000000 & & & & \\
\hline $\mathrm{C}$ & 2.868907000000 & 0.053135000000 & -0.216485000000 & & & & \\
\hline $\mathrm{H}$ & 0.633185000000 & -2.501359000000 & 0.071727000000 & & & & \\
\hline $\mathrm{H}$ & 3.073354000000 & -2.090076000000 & -0.282062000000 & & & & \\
\hline $\mathrm{H}$ & 3.924027000000 & 0.241783000000 & -0.382029000000 & & & & \\
\hline $\mathrm{Li}$ & -3.236534000000 & -1.273944000000 & -0.892970000000 & & & & \\
\hline
\end{tabular}


Table S14. Optimized geometries of stationary points of interaction of lithium enolate 24 and HCN. PCM/B3LYP/6$311++\mathrm{G}(\mathrm{d}, \mathrm{p})$ calculations (Benzene, $\left.25^{\circ} \mathrm{C}\right)$. Coordinates are in angstroms. Sum of electronic and thermal Free Energies in a.u. are in parentheses.

\begin{tabular}{|c|c|c|c|c|c|c|c|}
\hline \multicolumn{4}{|c|}{$\begin{array}{c}24 \\
E_{\text {tot. }}=-391.94340 \text { a.u., } v_{\text {im. }}=0(-391.85139)\end{array}$} & \multicolumn{4}{|c|}{$\begin{array}{c}\text { TS1 of exo-1 path } \\
E_{\text {tot. }}=-485.40804 \text { a.u., } v_{\text {im. }}=-227.6 \mathrm{~cm}^{-1}(-485.30011)\end{array}$} \\
\hline $\mathrm{C}$ & -0.825064000000 & 0.990867000000 & 0.015361000000 & $\mathrm{C}$ & -0.520455000000 & 0.815566000000 & -0.632167000000 \\
\hline $\mathrm{C}$ & 0.377645000000 & 0.125864000000 & 0.033413000000 & $\mathrm{C}$ & -0.124641000000 & -0.570493000000 & -0.450000000000 \\
\hline C & -0.829415000000 & 2.340191000000 & 0.202449000000 & C & 0.356119000000 & 1.825227000000 & -1.024826000000 \\
\hline $\mathrm{C}$ & 1.650176000000 & 0.647118000000 & -0.153917000000 & $\mathrm{C}$ & 1.131736000000 & -1.039428000000 & -0.917749000000 \\
\hline $\mathrm{H}$ & -1.752318000000 & 2.902075000000 & 0.113666000000 & $\mathrm{H}$ & -0.034296000000 & 2.827315000000 & -1.172931000000 \\
\hline $\mathrm{H}$ & 0.062322000000 & 2.901356000000 & 0.451338000000 & $\mathrm{H}$ & 1.296791000000 & 1.611504000000 & -1.515462000000 \\
\hline $\mathrm{H}$ & 1.723347000000 & 1.713836000000 & -0.398439000000 & $\mathrm{H}$ & 1.604013000000 & -0.466787000000 & -1.729894000000 \\
\hline $\mathrm{O}$ & 2.763308000000 & -0.000147000000 & -0.093955000000 & $\mathrm{O}$ & 1.750549000000 & -2.056800000000 & -0.481729000000 \\
\hline $\mathrm{C}$ & -2.092321000000 & 0.288280000000 & -0.176649000000 & $\mathrm{C}$ & -1.857889000000 & 1.150487000000 & -0.207596000000 \\
\hline $\mathrm{C}$ & 0.198409000000 & -1.296794000000 & 0.170350000000 & $\mathrm{C}$ & -0.995670000000 & -1.473420000000 & 0.231779000000 \\
\hline C & -1.026303000000 & -1.884280000000 & 0.109898000000 & $\mathrm{C}$ & -2.244703000000 & -1.094085000000 & 0.643986000000 \\
\hline $\mathrm{H}$ & -2.982659000000 & 0.893513000000 & -0.318209000000 & $\mathrm{H}$ & -2.202024000000 & 2.166854000000 & -0.371629000000 \\
\hline $\mathrm{C}$ & -2.191323000000 & -1.062100000000 & -0.112418000000 & $\mathrm{H}$ & 1.685187000000 & 2.852387000000 & 1.035142000000 \\
\hline $\mathrm{H}$ & 1.090231000000 & -1.904232000000 & 0.279554000000 & C & 1.752156000000 & 1.789003000000 & 0.908076000000 \\
\hline $\mathrm{H}$ & -1.134529000000 & -2.960081000000 & 0.191015000000 & $\mathrm{~N}$ & 2.149461000000 & 0.725848000000 & 1.190645000000 \\
\hline $\mathrm{H}$ & -3.161103000000 & -1.537987000000 & -0.220761000000 & C & -2.677863000000 & 0.243276000000 & 0.401302000000 \\
\hline \multirow[t]{4}{*}{$\mathrm{Li}$} & 4.159141000000 & -0.967393000000 & 0.040850000000 & $\mathrm{H}$ & -0.658453000000 & -2.495963000000 & 0.363225000000 \\
\hline & & & & $\mathrm{H}$ & -2.913140000000 & -1.804120000000 & 1.116822000000 \\
\hline & & & & $\mathrm{H}$ & -3.677001000000 & 0.541617000000 & 0.702725000000 \\
\hline & & & & $\mathrm{Li}$ & 2.311860000000 & -1.245378000000 & 1.124831000000 \\
\hline \multicolumn{4}{|c|}{ Product of exo-1 path } & \multicolumn{4}{|c|}{$\mathrm{HCN}$} \\
\hline \multicolumn{4}{|c|}{$E_{\text {tot. }}=-485.46185$ a.u., $v_{\text {im. }}=0(-485.34484)$} & \multicolumn{4}{|c|}{$E_{\text {tot. }}=-93.45779$ a.u., $v_{\text {im. }}=0(-93.46074)$} \\
\hline $\mathrm{C}$ & 0.621221000000 & 0.889820000000 & 0.155766000000 & $\mathrm{~N}$ & 0.000000000000 & 0.000000000000 & 0.650827000000 \\
\hline C & 0.146096000000 & -0.425463000000 & 0.207450000000 & $\mathrm{C}$ & 0.000000000000 & 0.000000000000 & -0.498241000000 \\
\hline $\mathrm{C}$ & -0.391037000000 & 2.004503000000 & 0.295728000000 & $\mathrm{H}$ & 0.000000000000 & 0.000000000000 & -1.566343000000 \\
\hline $\mathrm{C}$ & -1.341008000000 & -0.660642000000 & 0.464474000000 & & & & \\
\hline $\mathrm{H}$ & -0.033489000000 & 2.935341000000 & -0.150306000000 & & & & \\
\hline $\mathrm{H}$ & -0.576984000000 & 2.219912000000 & 1.359318000000 & & & & \\
\hline $\mathrm{H}$ & -1.517728000000 & -0.408121000000 & 1.536460000000 & & & & \\
\hline 0 & -1.815496000000 & -1.879746000000 & 0.125461000000 & & & & \\
\hline C & 1.979128000000 & 1.130612000000 & -0.063598000000 & & & & \\
\hline $\mathrm{C}$ & 1.029562000000 & -1.490388000000 & 0.038500000000 & & & & \\
\hline C & 2.387750000000 & -1.248195000000 & -0.163847000000 & & & & \\
\hline $\mathrm{H}$ & 2.345293000000 & 2.151184000000 & -0.121791000000 & & & & \\
\hline $\mathrm{H}$ & -2.288249000000 & 2.350092000000 & -0.846142000000 & & & & \\
\hline C & -1.704333000000 & 1.588455000000 & -0.323996000000 & & & & \\
\hline $\mathrm{N}$ & -2.153298000000 & 0.402937000000 & -0.278416000000 & & & & \\
\hline C & 2.863937000000 & 0.063013000000 & -0.213173000000 & & & & \\
\hline $\mathrm{H}$ & 0.630671000000 & -2.497343000000 & 0.064240000000 & & & & \\
\hline $\mathrm{H}$ & 3.074495000000 & -2.078810000000 & -0.287497000000 & & & & \\
\hline $\mathrm{H}$ & 3.918874000000 & 0.254596000000 & -0.376519000000 & & & & \\
\hline $\mathrm{Li}$ & -3.167909000000 & -1.273246000000 & -0.872121000000 & & & & \\
\hline
\end{tabular}




\section{NMR Spectra}

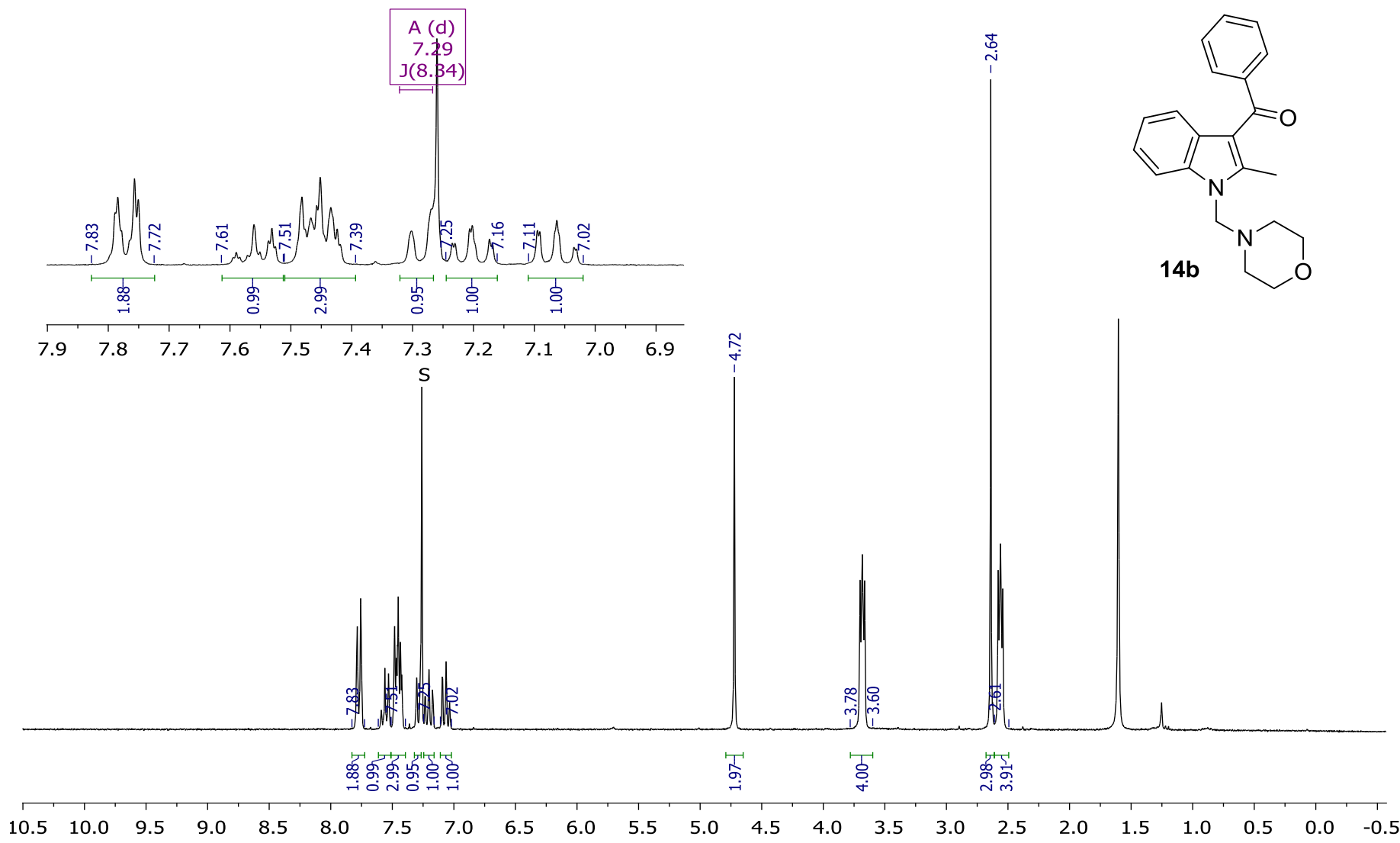

${ }^{1} \mathrm{H} \mathrm{NMR}, \mathrm{CDCl}_{3}, 250 \mathrm{MHz}$
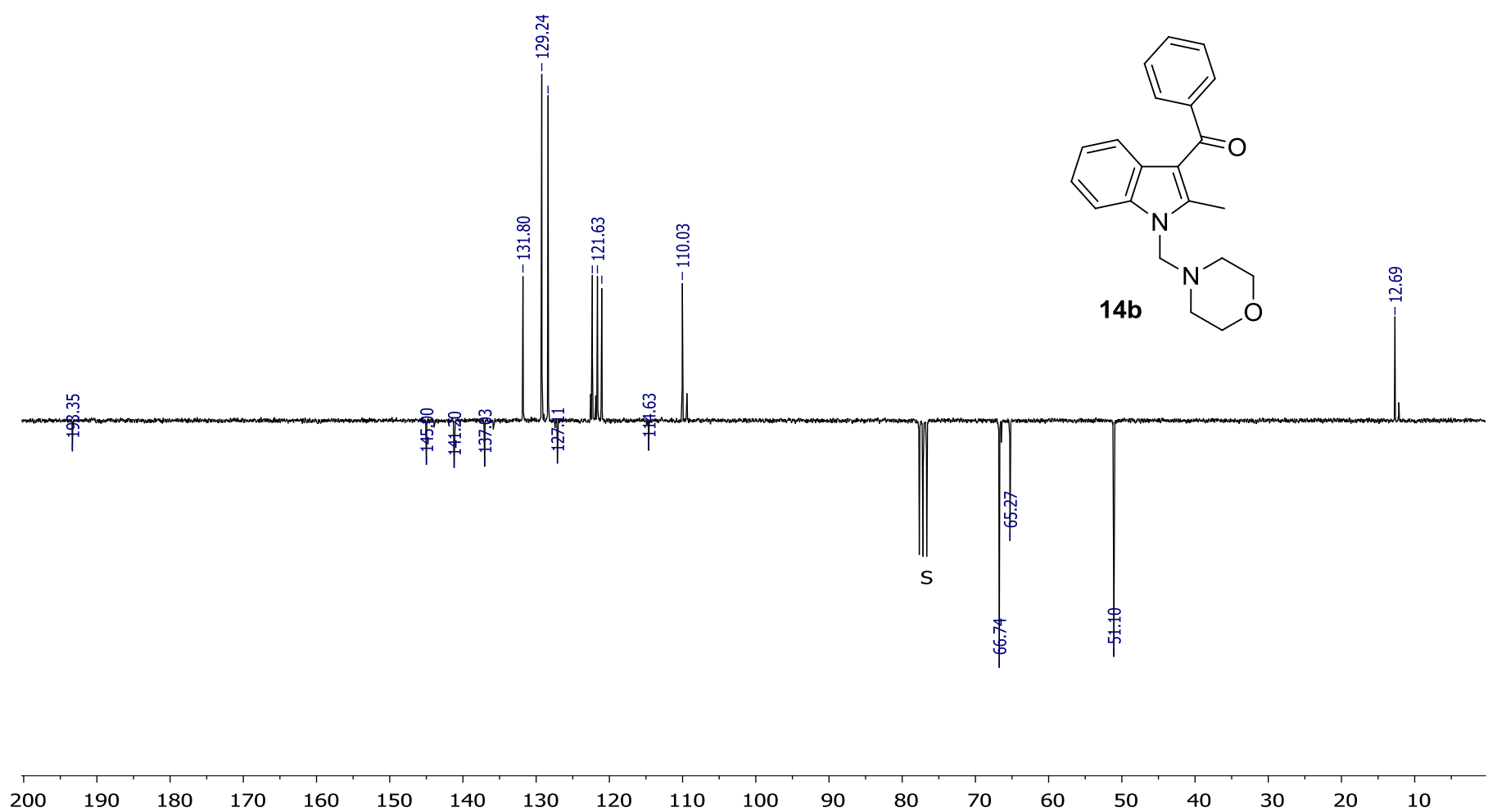

${ }^{13} \mathrm{C} \mathrm{NMR}, \mathrm{CDCl}_{3}, 62.9 \mathrm{MHz}$ 

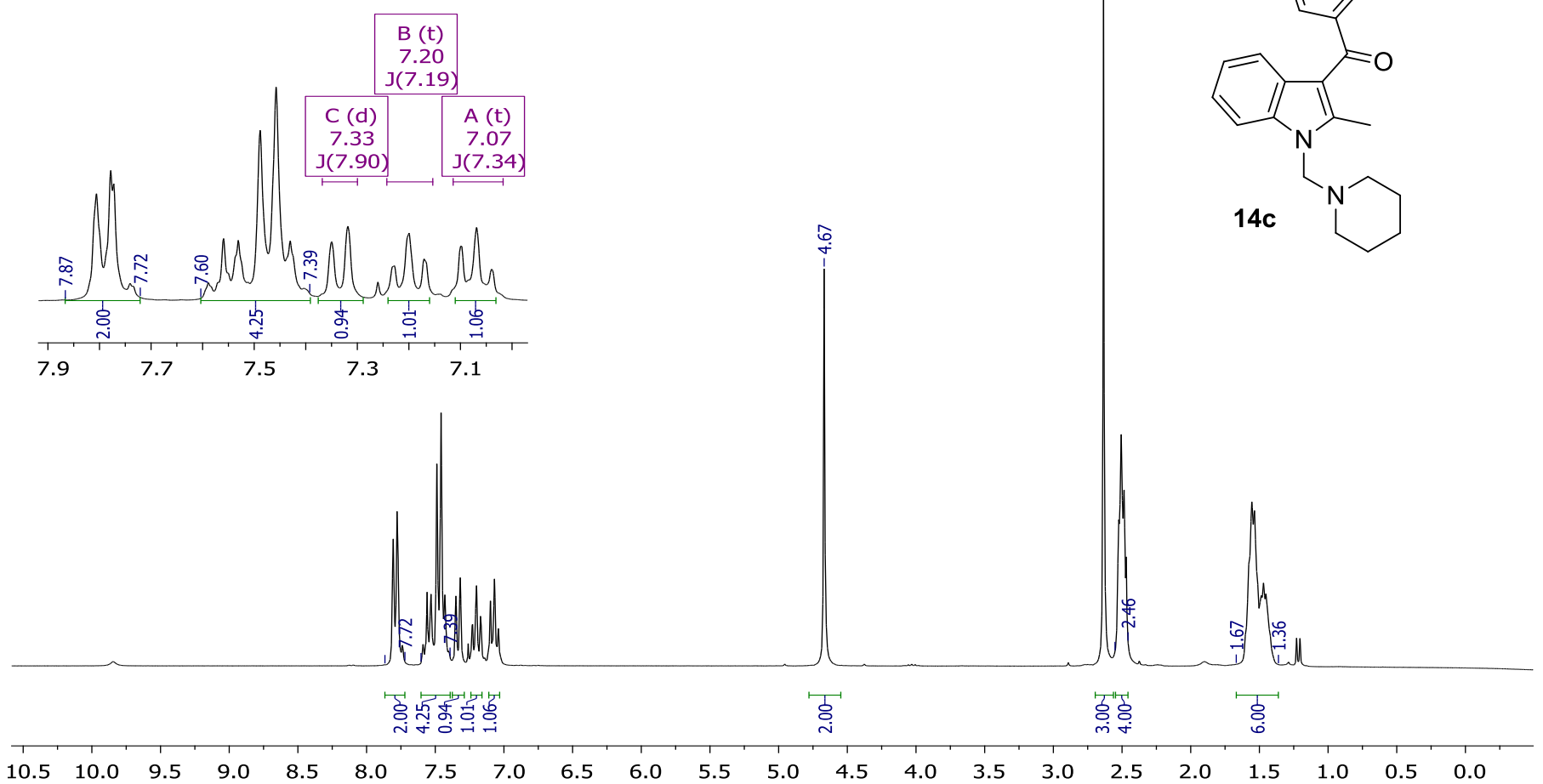

${ }^{1} \mathrm{H} \mathrm{NMR}, \mathrm{CDCl}_{3}, 250 \mathrm{MHz}$

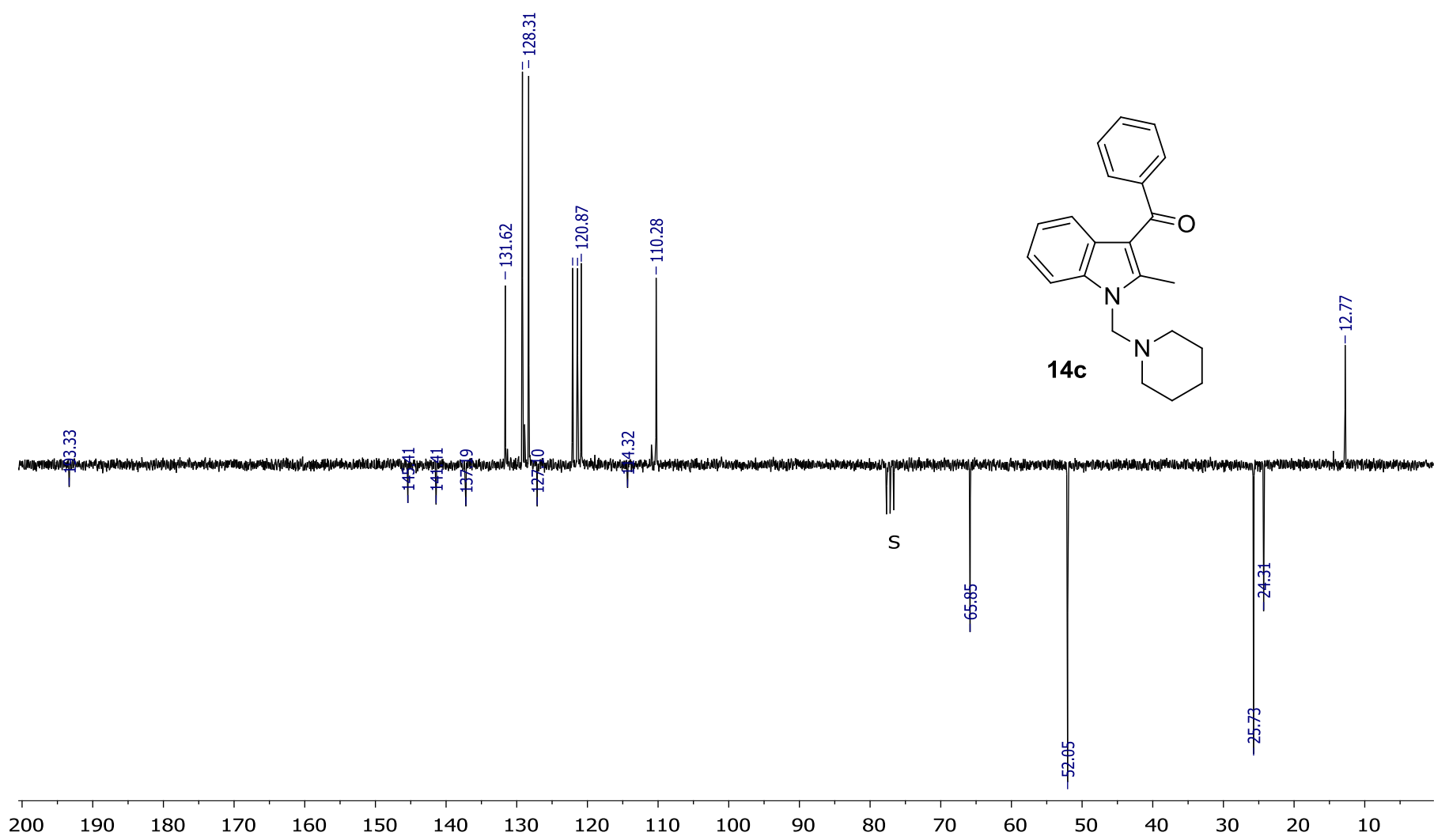

${ }^{13} \mathrm{C} \mathrm{NMR}, \mathrm{CDCl}_{3}, 62.9 \mathrm{MHz}$ 

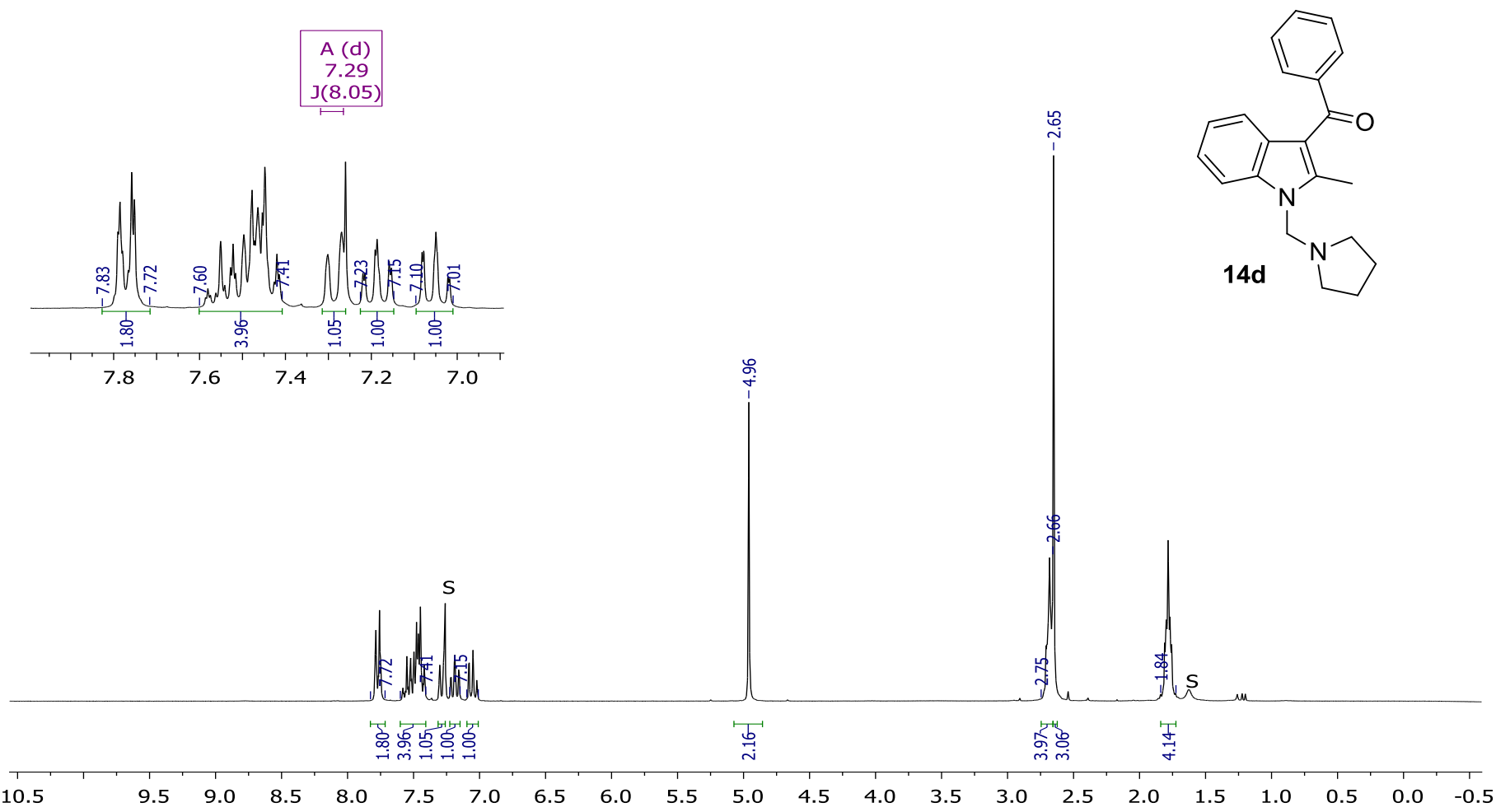

${ }^{1} \mathrm{H} \mathrm{NMR}, \mathrm{CDCl}_{3}, 250 \mathrm{MHz}$

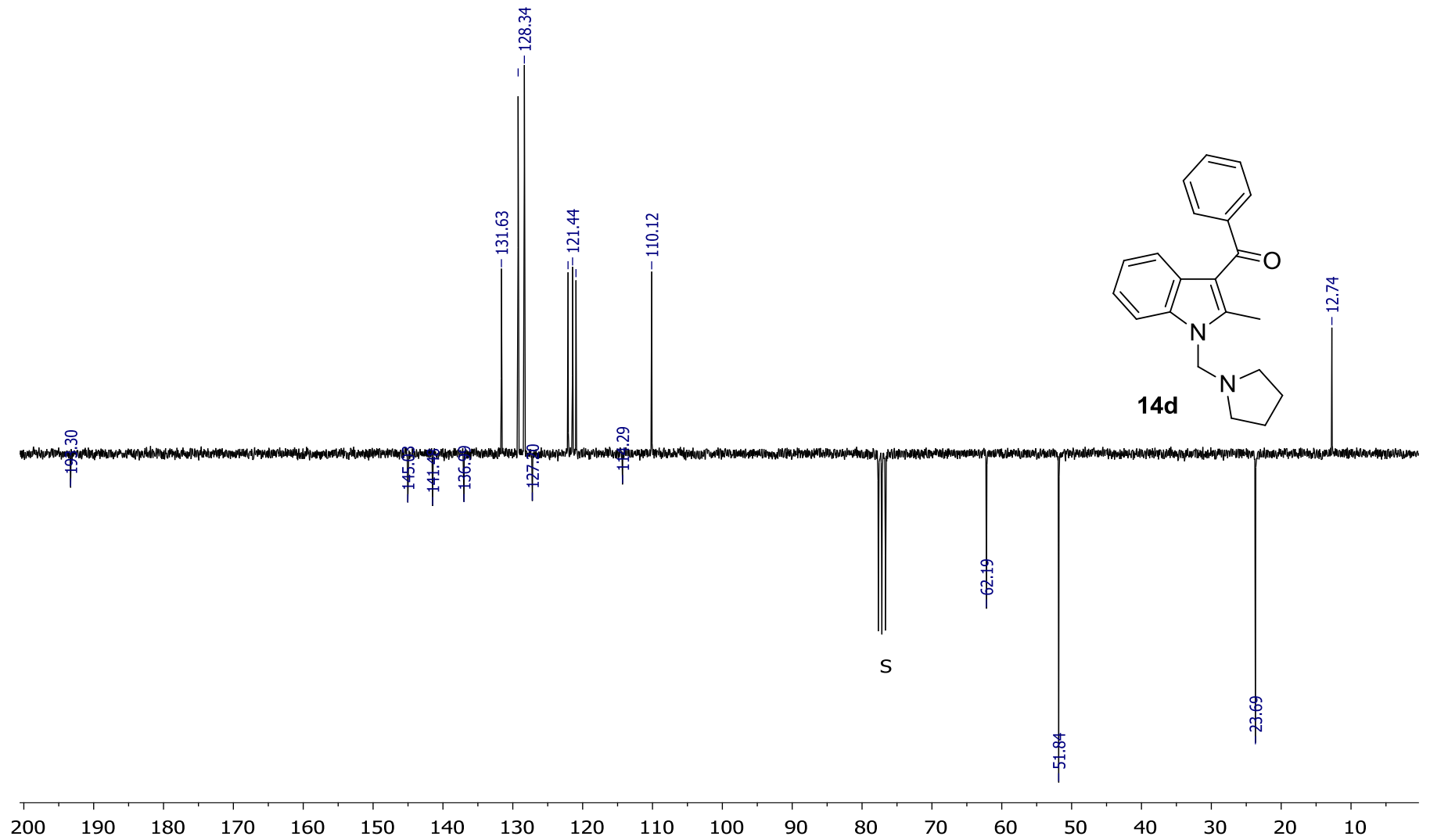

${ }^{13} \mathrm{C} \mathrm{NMR}, \mathrm{CDCl}_{3}, 62.9 \mathrm{MHz}$ 


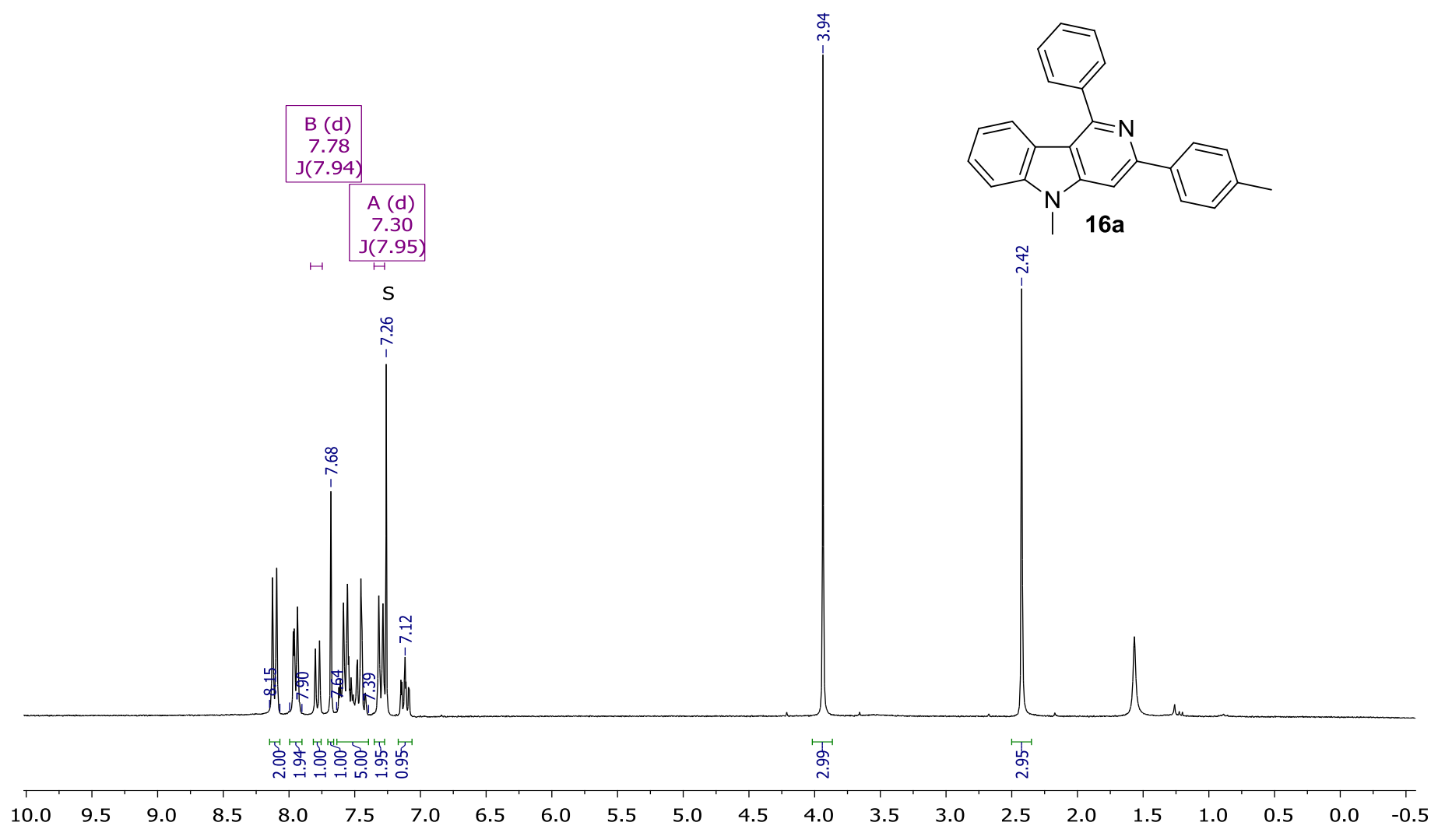

\section{${ }^{1} \mathrm{H} \mathrm{NMR}, \mathrm{CDCl}_{3}, 250 \mathrm{MHz}$}

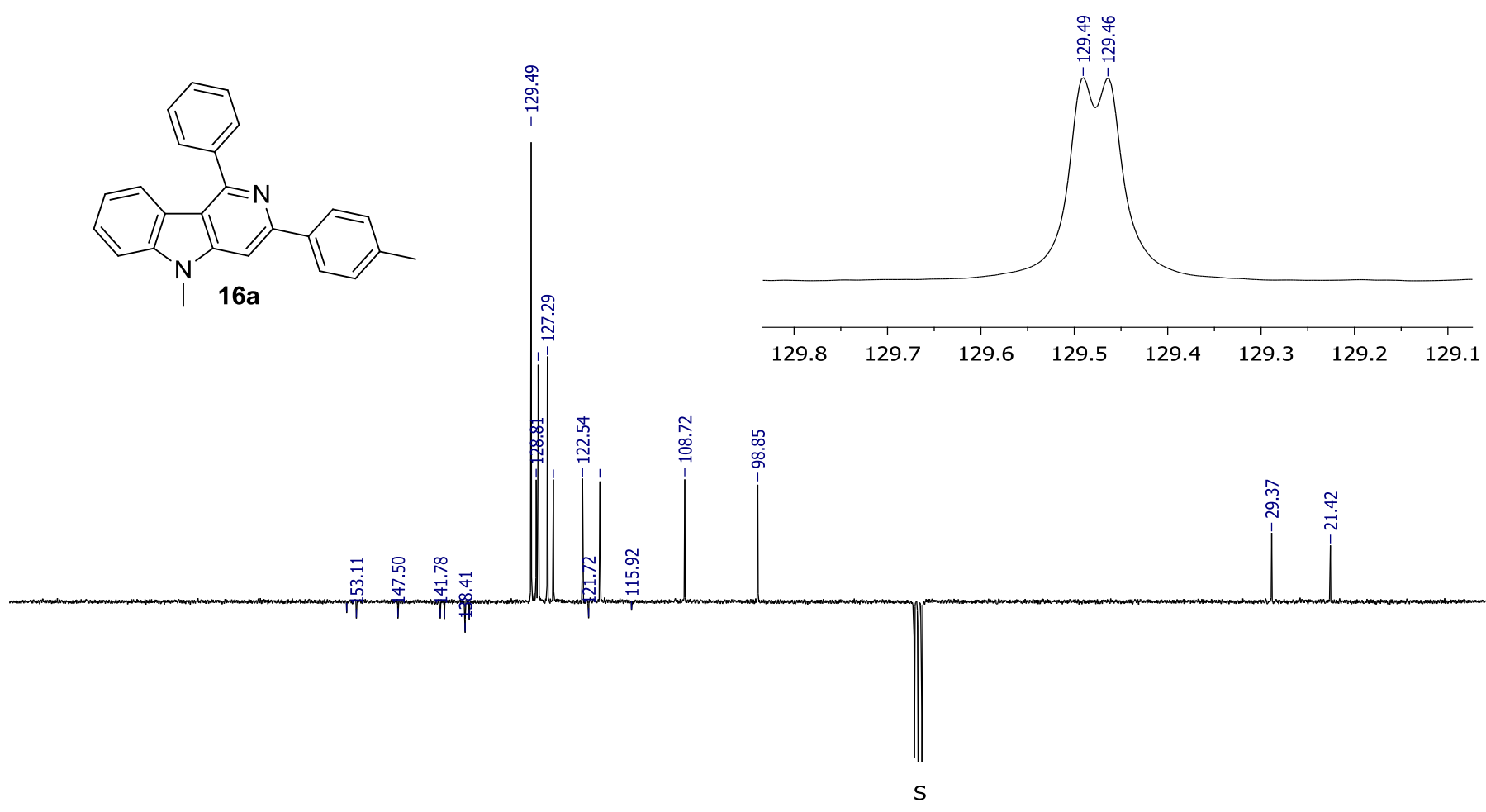

$\begin{array}{lllllllllllllllllll}190 & 180 & 170 & 160 & 150 & 140 & 130 & 120 & 110 & 100 & 90 & 80 & 70 & 60 & 50 & 40 & 30 & 20 & 10\end{array}$

${ }^{13} \mathrm{C} \mathrm{NMR}, \mathrm{CDCl}_{3}, 62.9 \mathrm{MHz}$ 

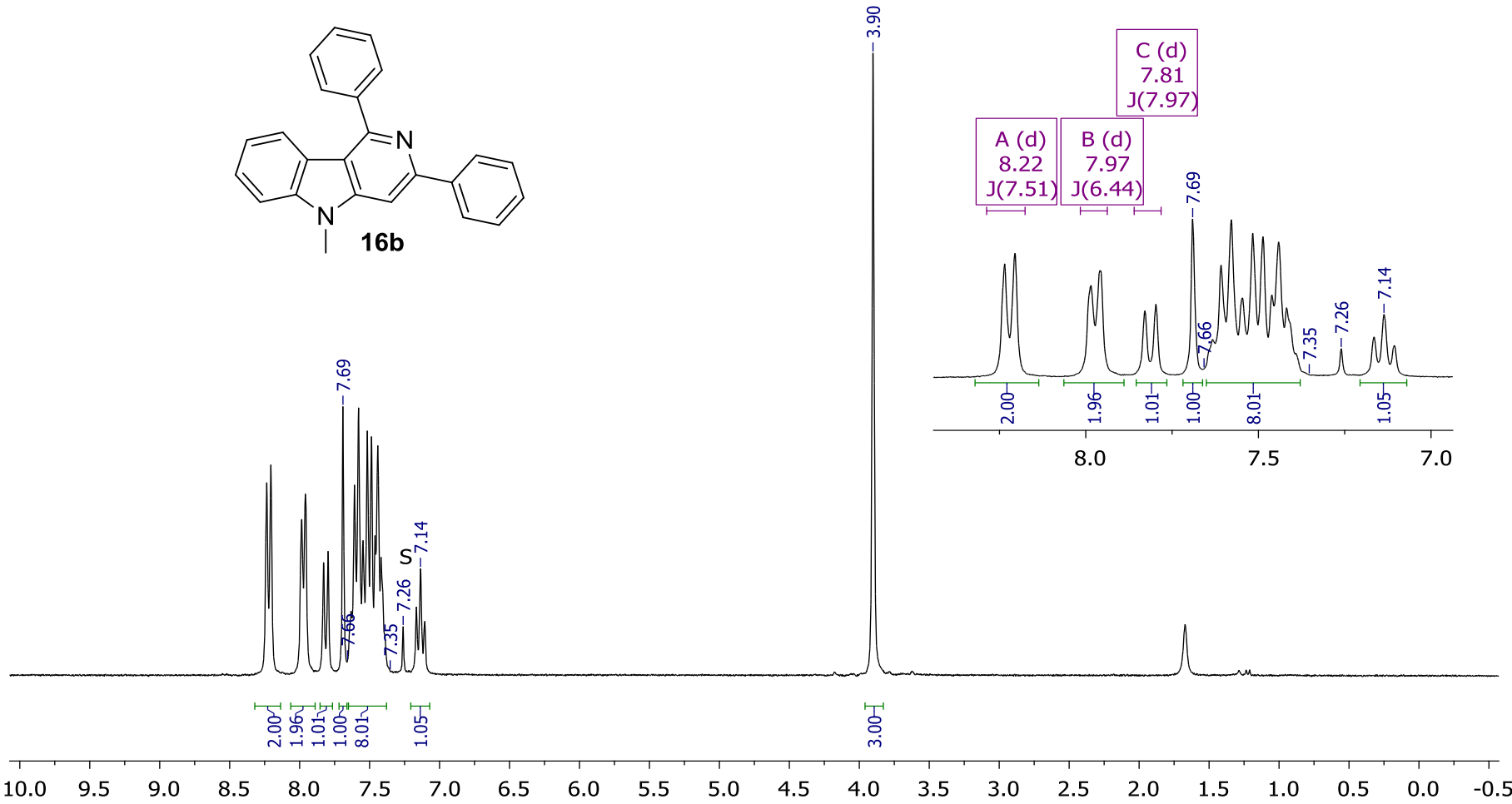

${ }^{1} \mathrm{H} \mathrm{NMR}, \mathrm{CDCl}_{3}, 250 \mathrm{MHz}$
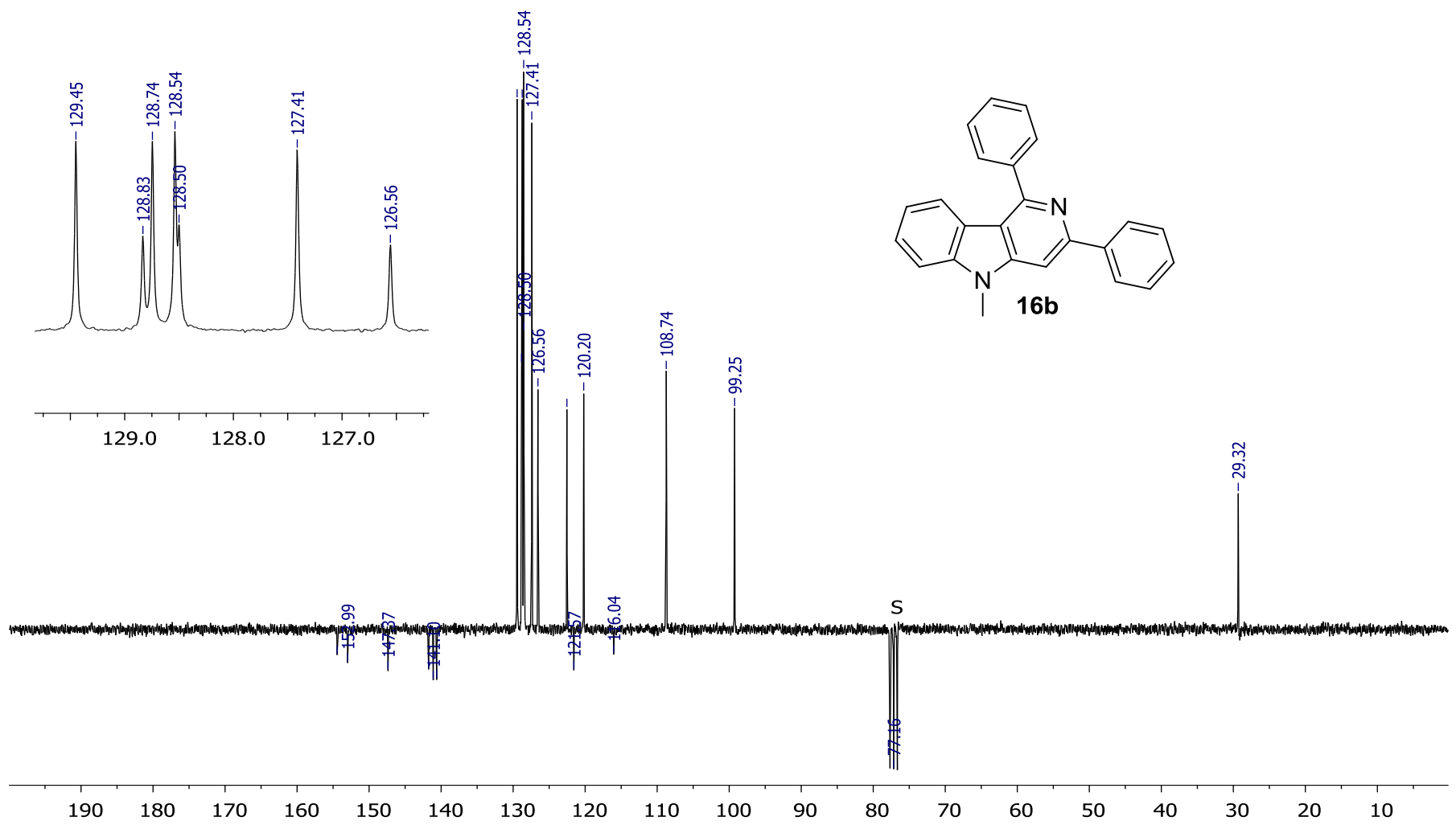

${ }^{13} \mathrm{C} \mathrm{NMR}, \mathrm{CDCl}_{3}, 62.9 \mathrm{MHz}$ 


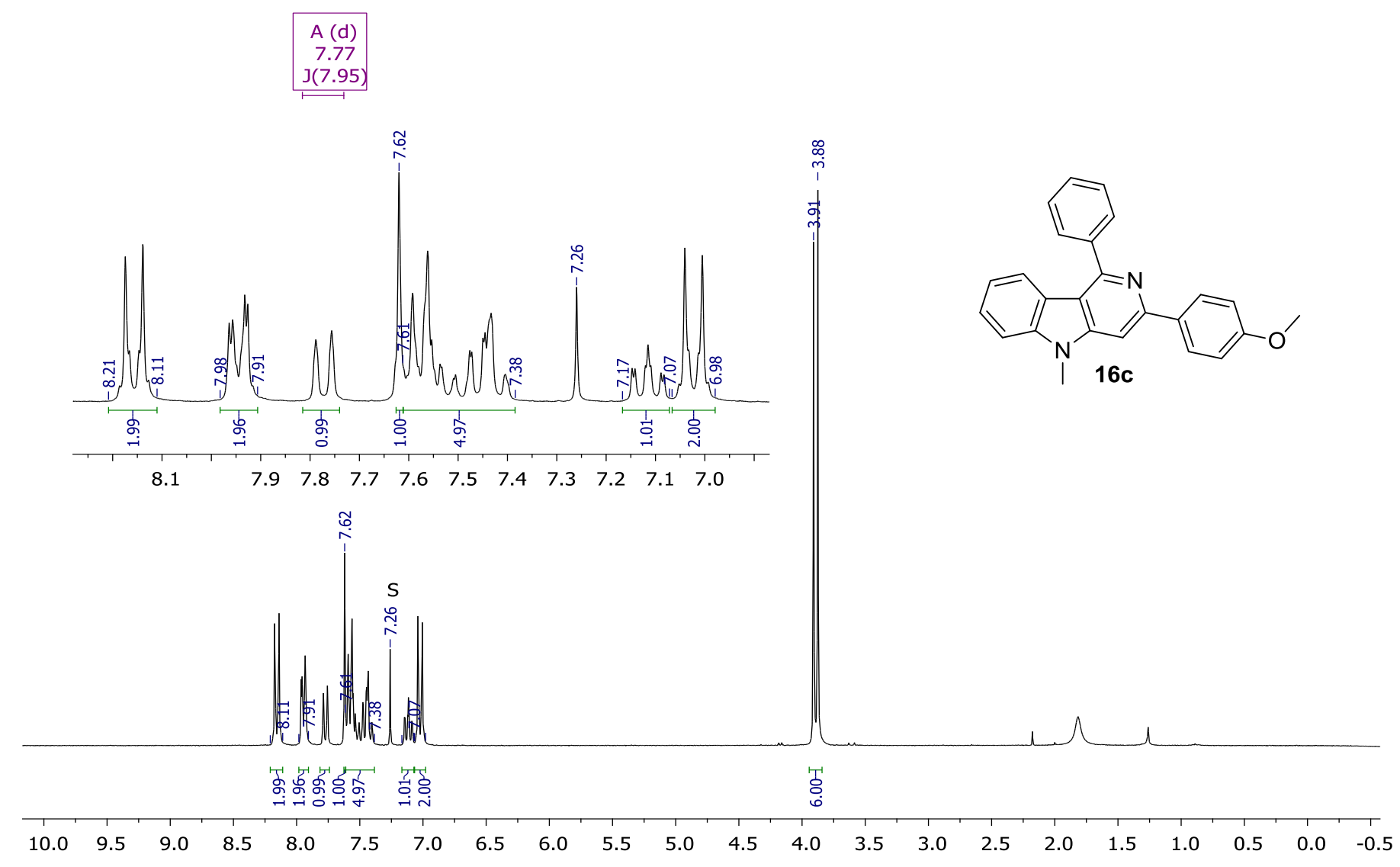

${ }^{1} \mathrm{H} \mathrm{NMR}, \mathrm{CDCl}_{3}, 250 \mathrm{MHz}$

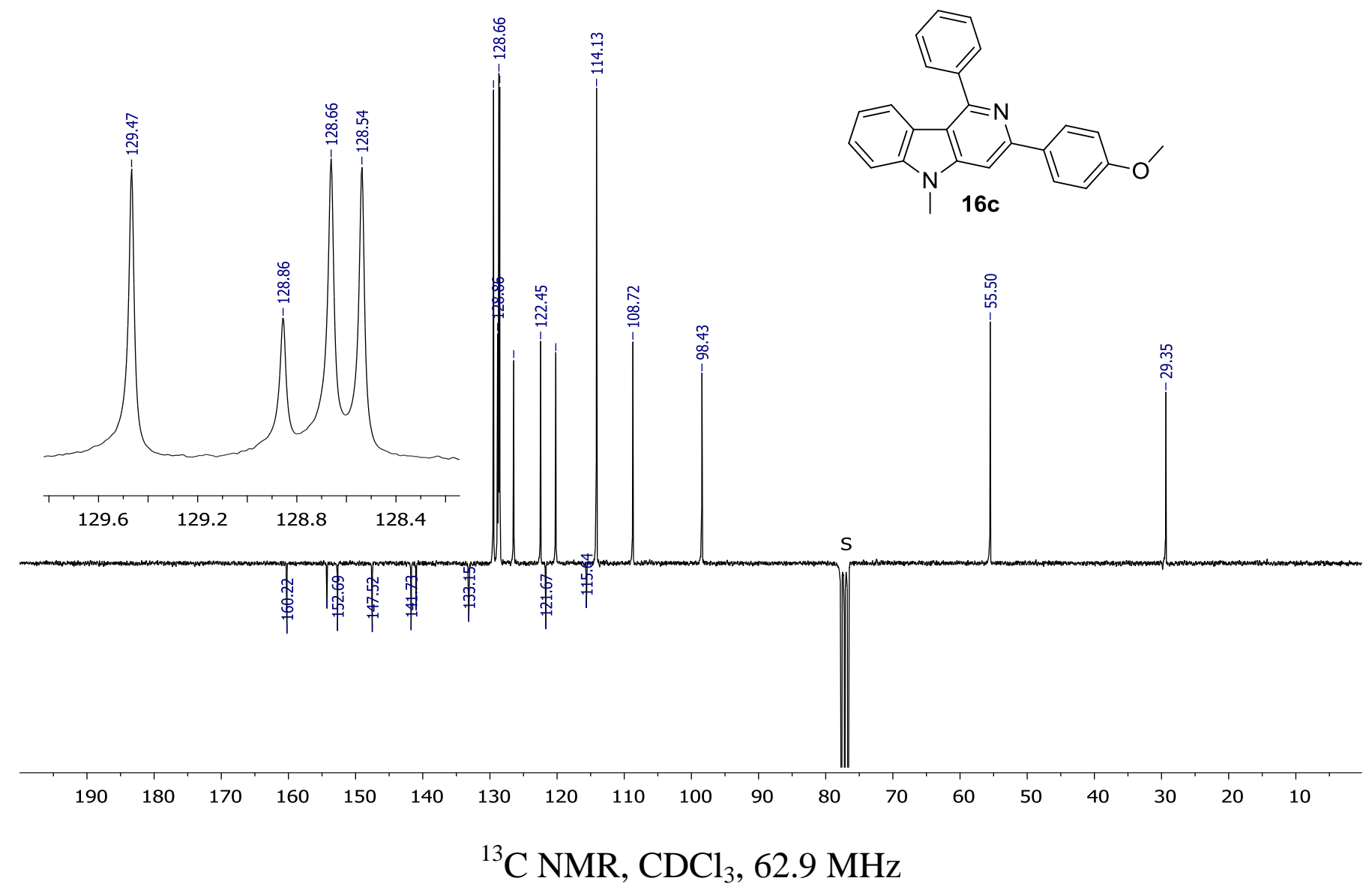



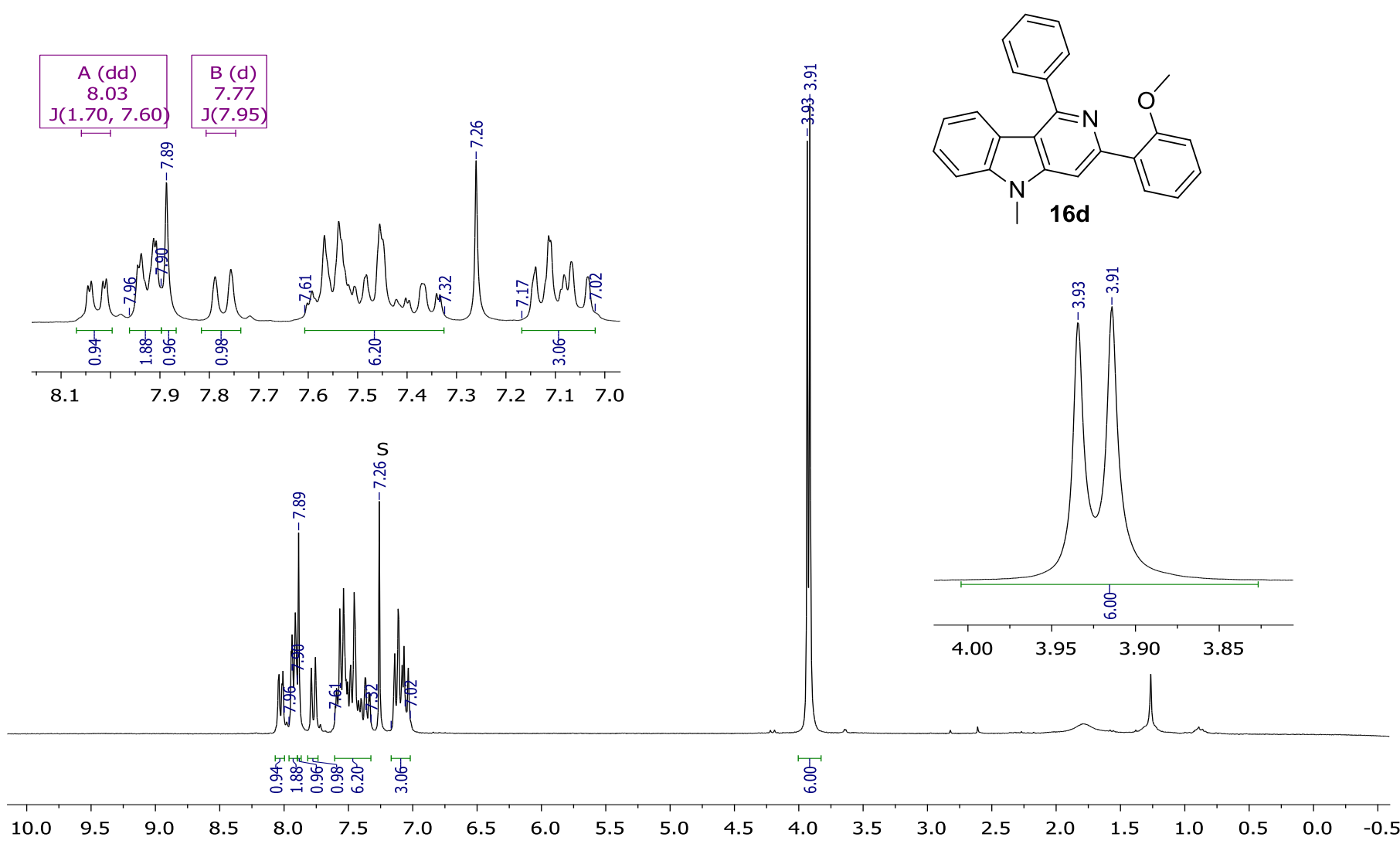

${ }^{1} \mathrm{H} \mathrm{NMR}, \mathrm{CDCl}_{3}, 250 \mathrm{MHz}$
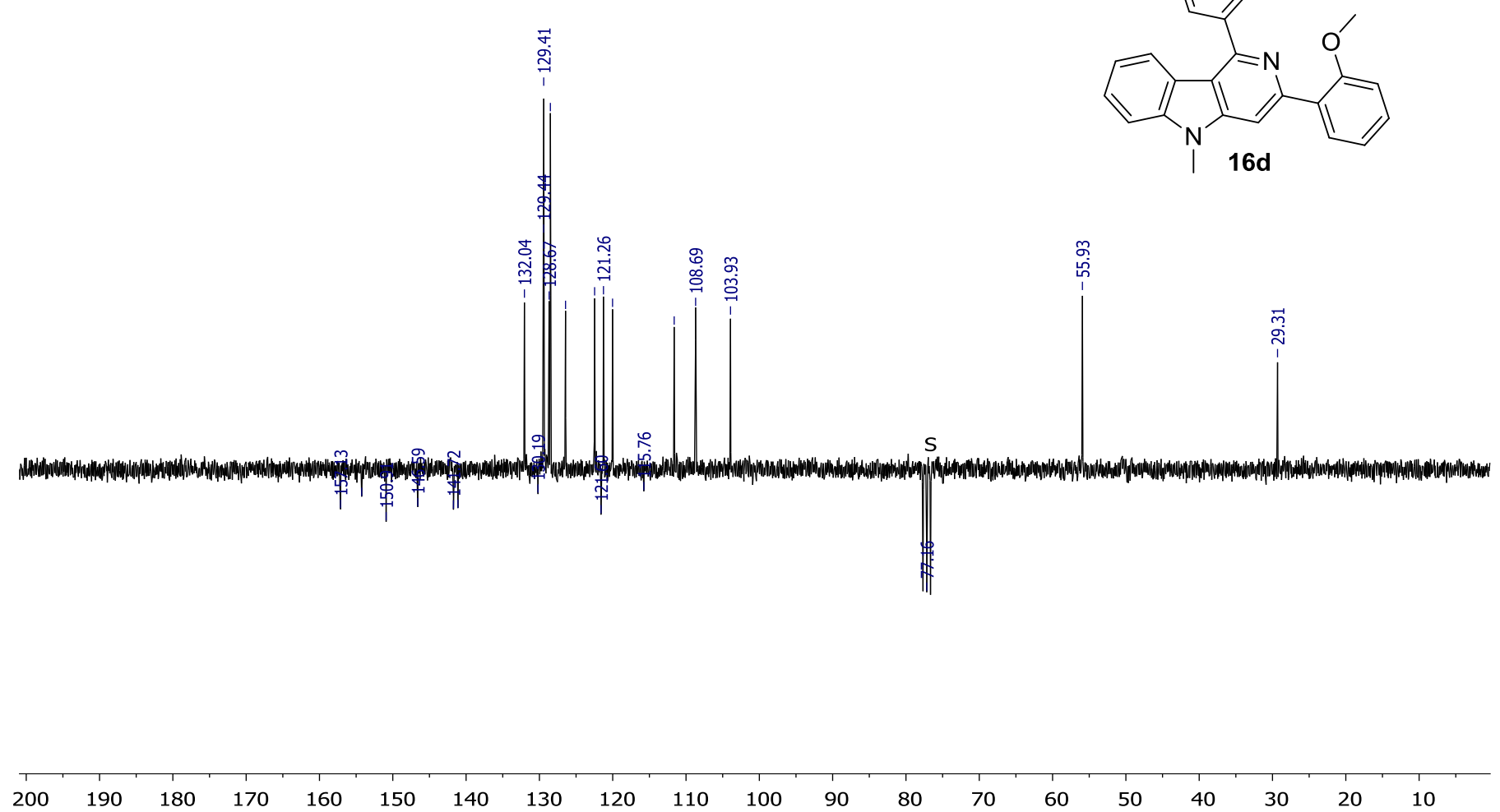

${ }^{13} \mathrm{C} \mathrm{NMR}, \mathrm{CDCl}_{3}, 62.9 \mathrm{MHz}$ 

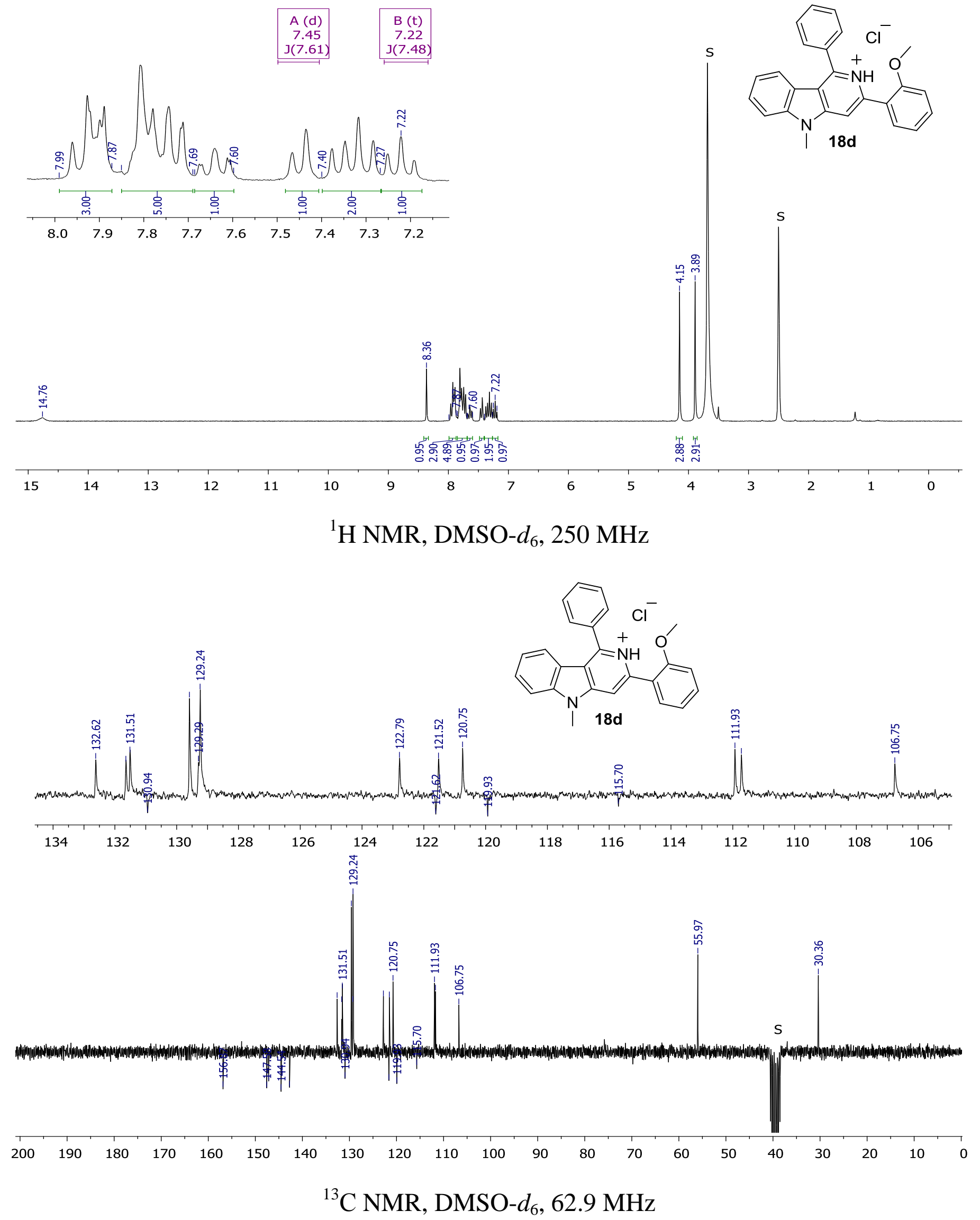


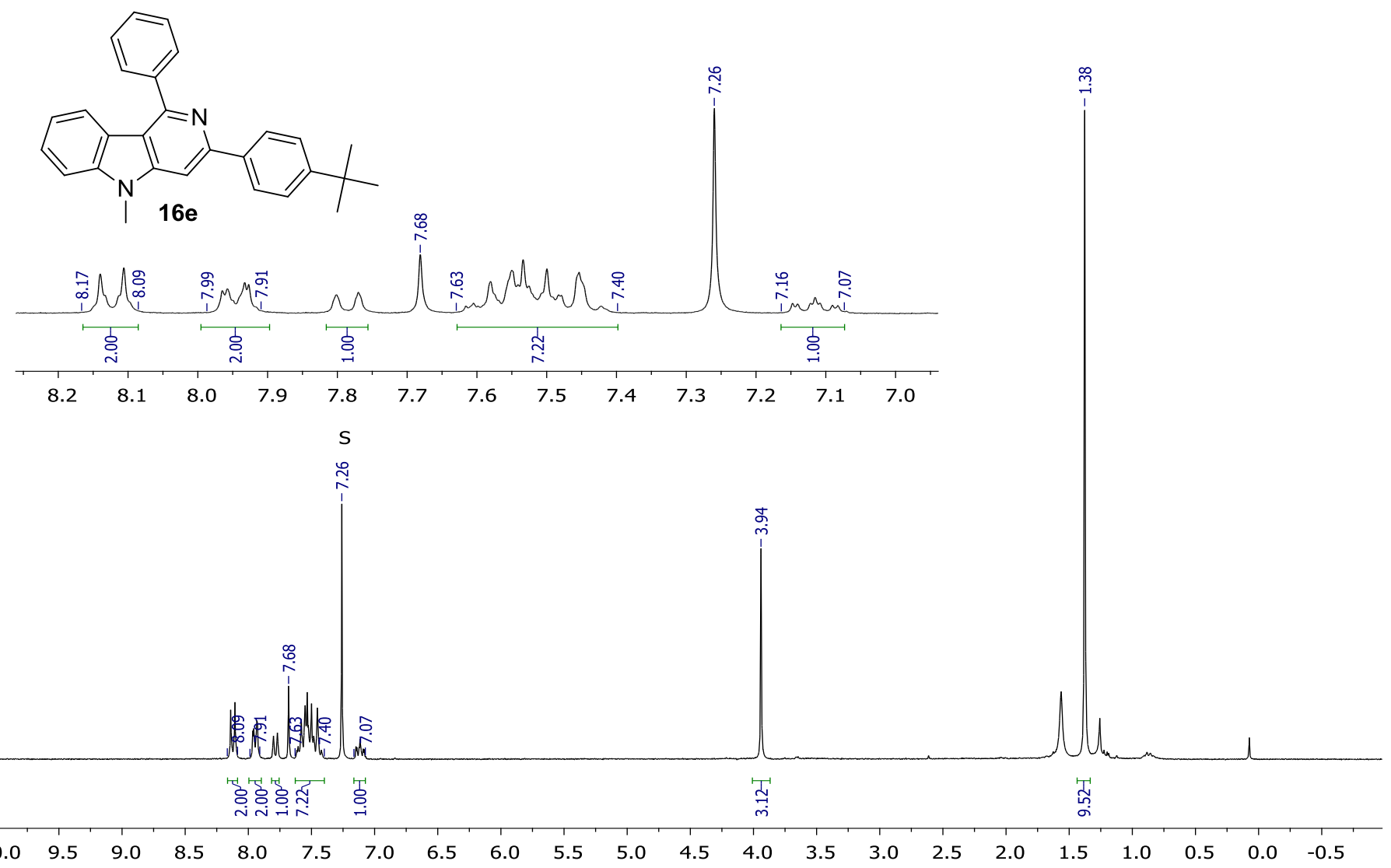

${ }^{1} \mathrm{H}$ NMR, $\mathrm{CDCl}_{3}, 250 \mathrm{MHz}$
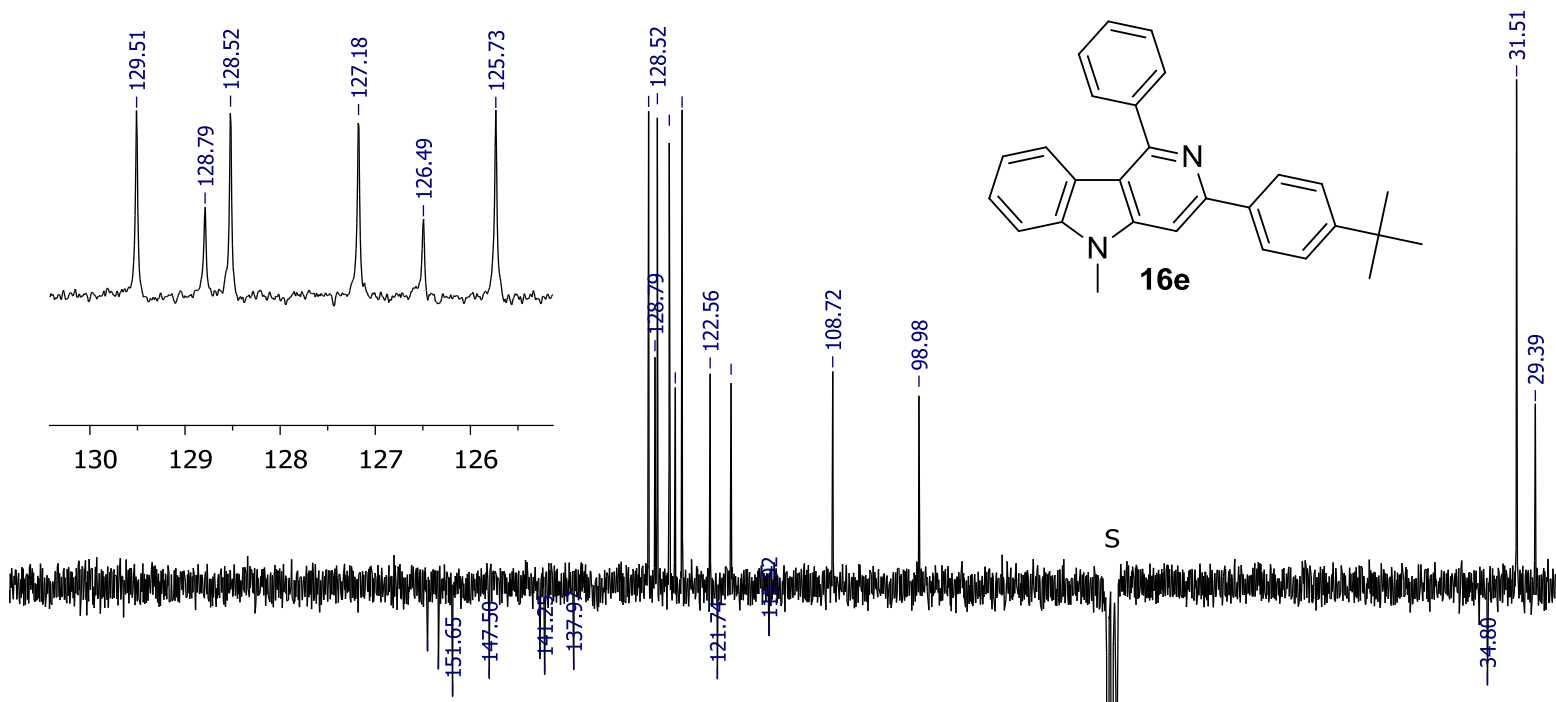

$\mathrm{S}$ 

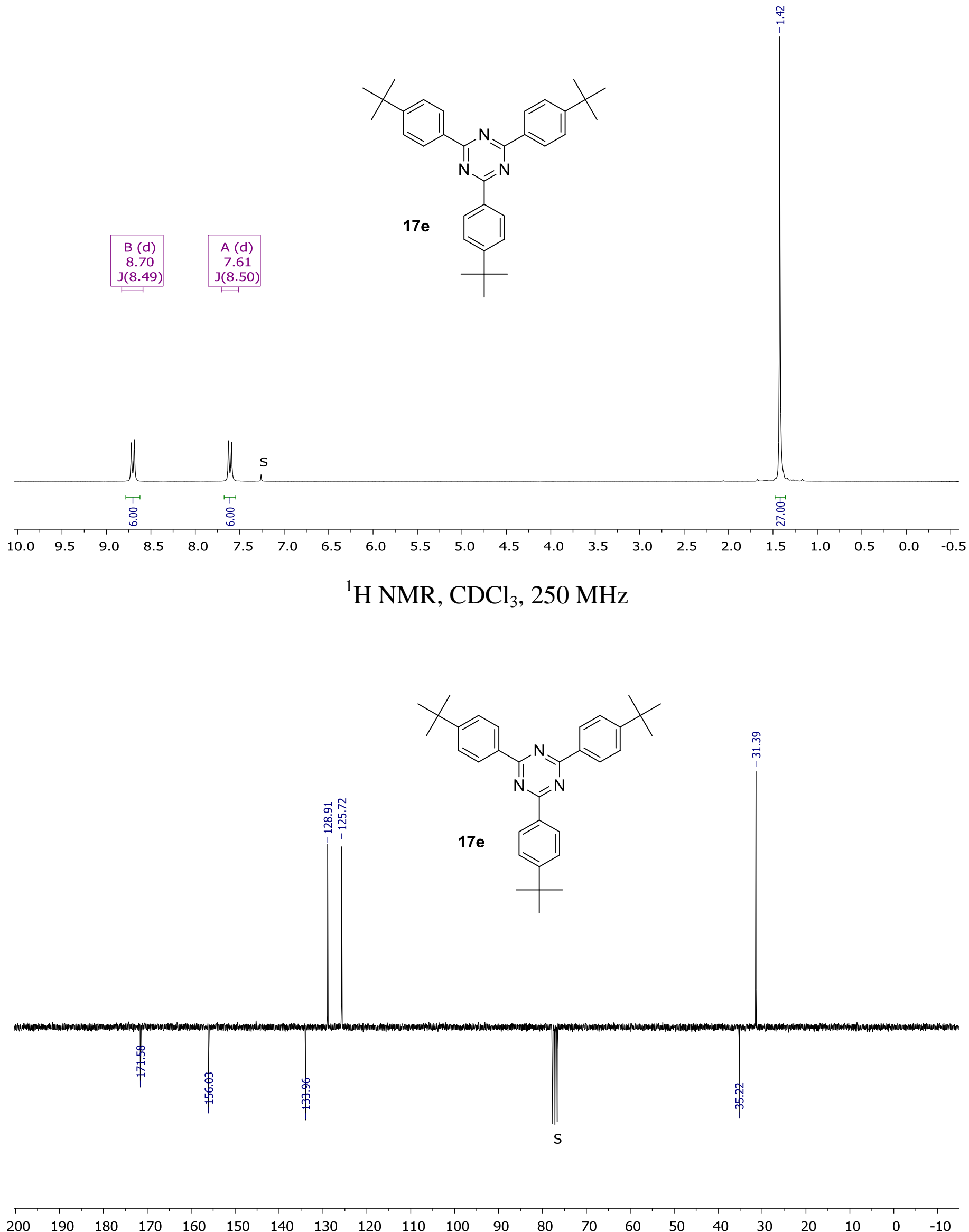

${ }^{13} \mathrm{C} \mathrm{NMR}, \mathrm{CDCl}_{3}, 62.9 \mathrm{MHz}$ 

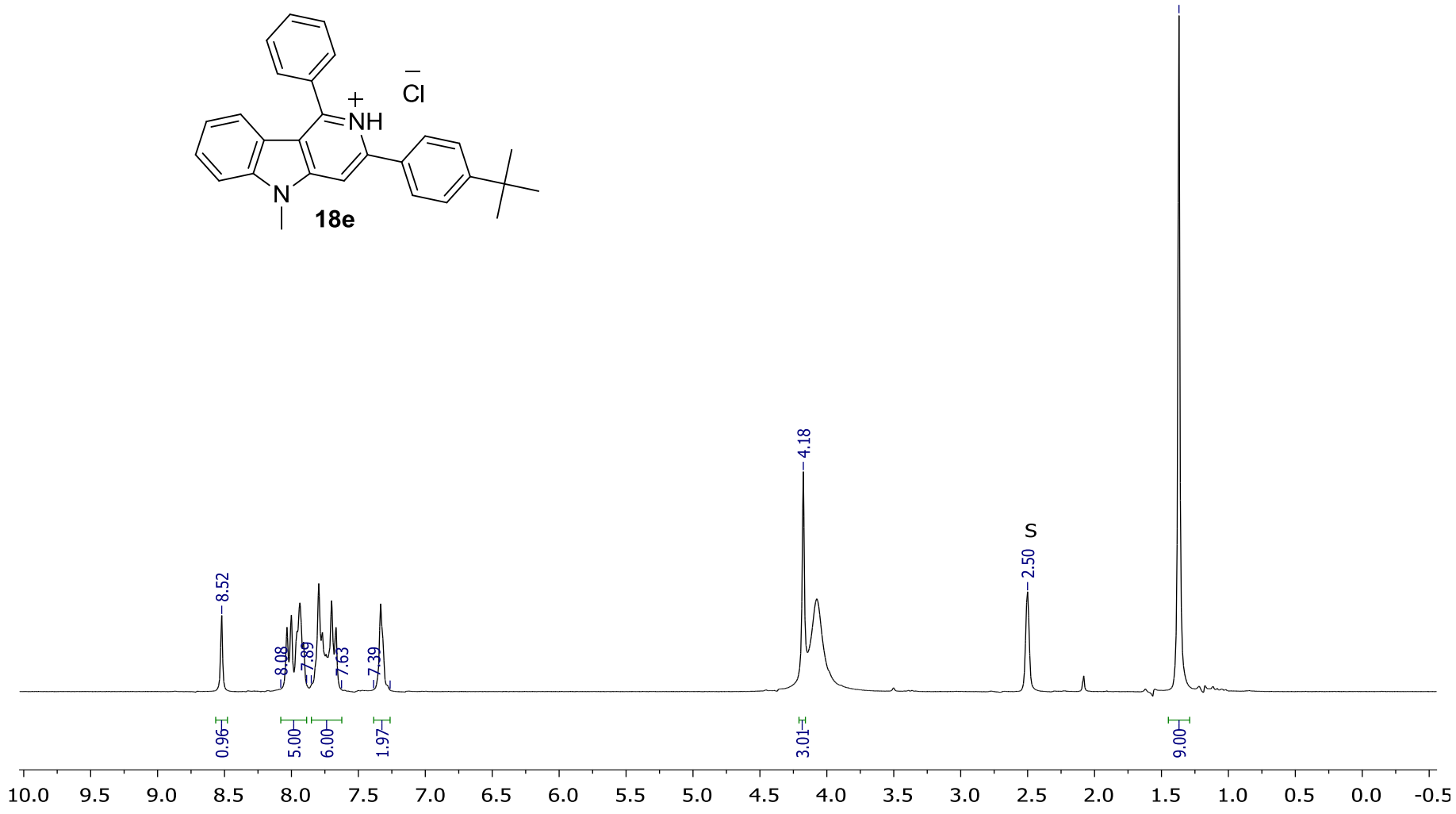

${ }^{1} \mathrm{H}$ NMR, DMSO- $d_{6}, 250 \mathrm{MHz}$
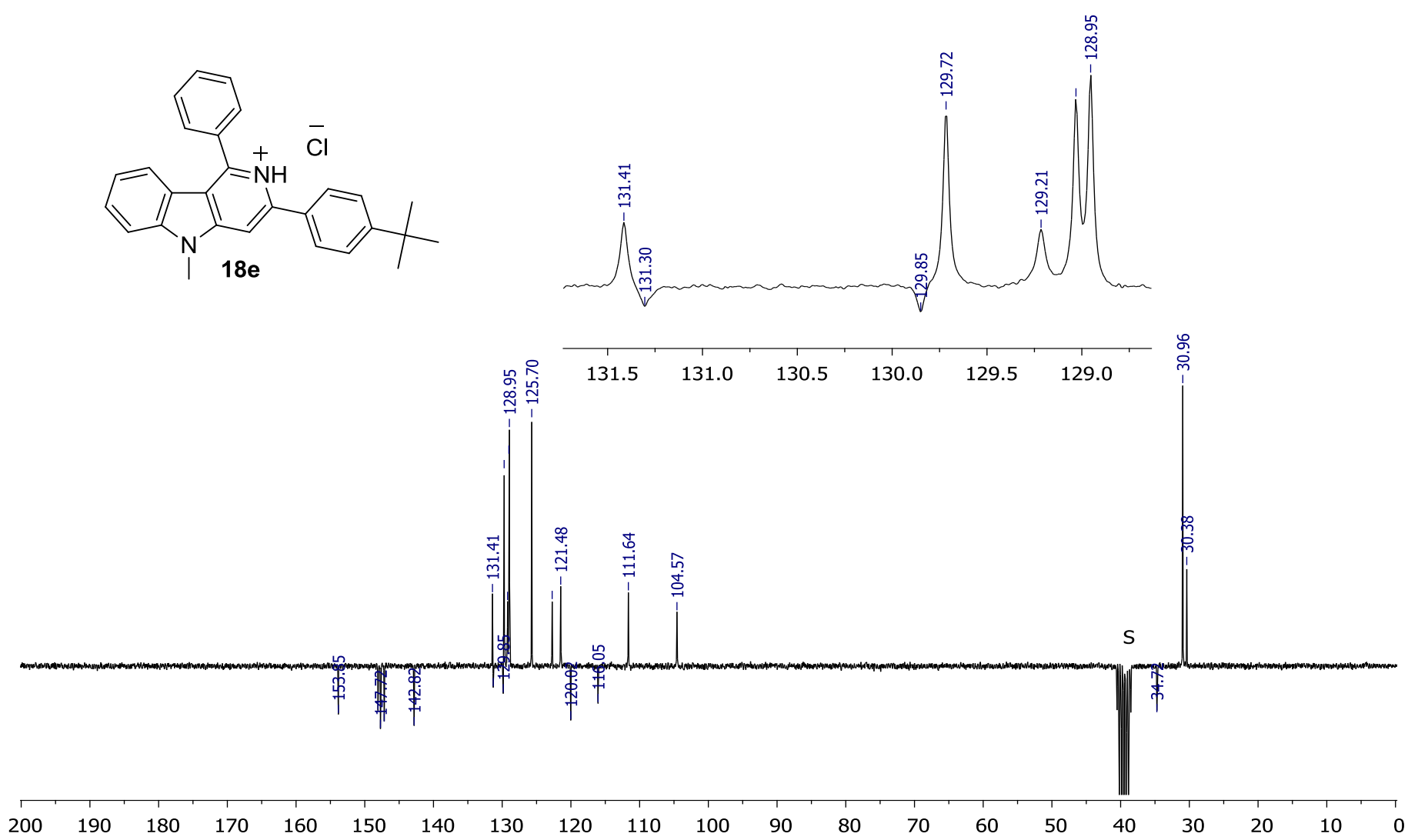

${ }^{13} \mathrm{C}$ NMR, DMSO- $d_{6}, 62.9 \mathrm{MHz}$ 


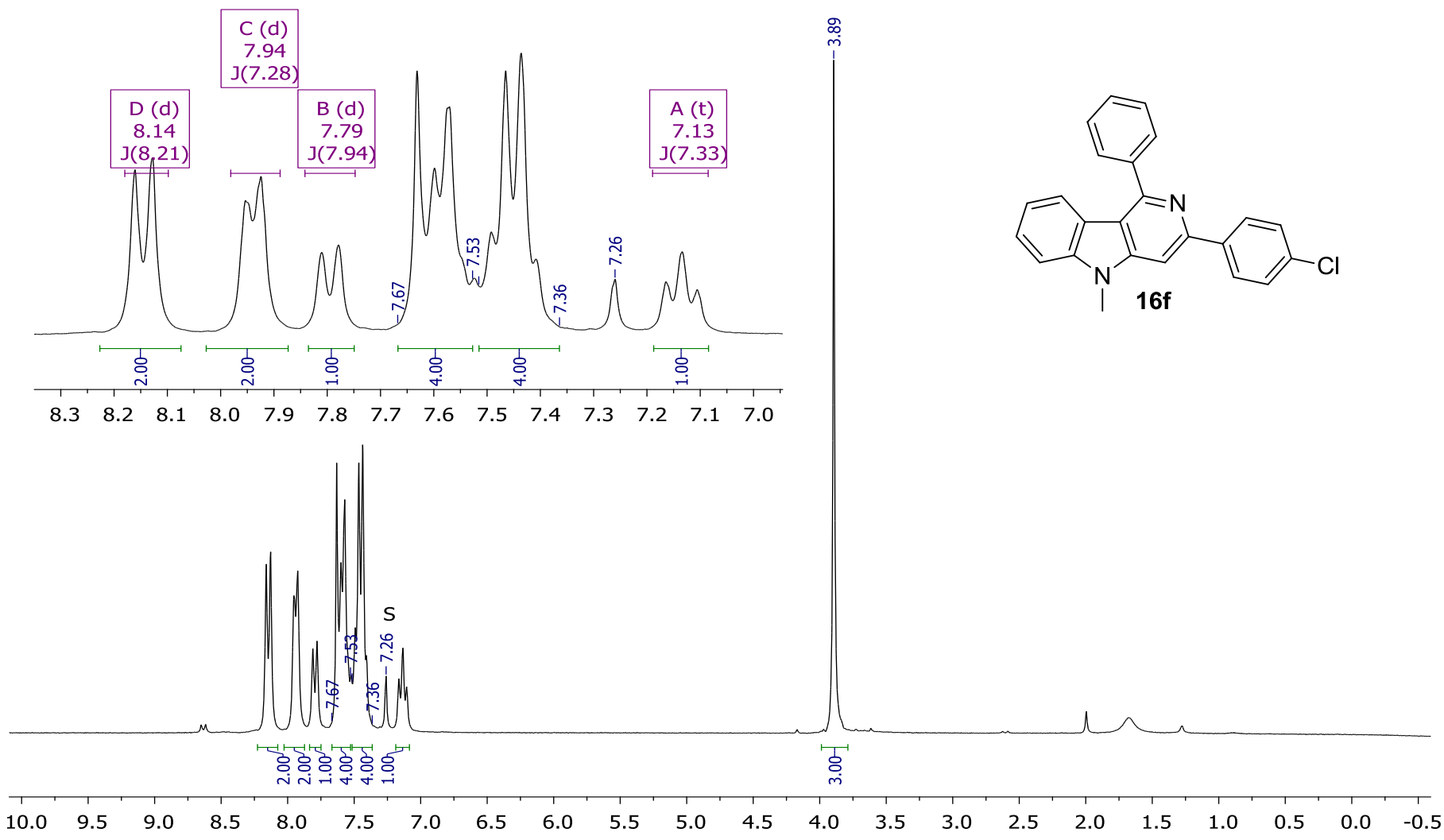

${ }^{1} \mathrm{H} \mathrm{NMR}, \mathrm{CDCl}_{3}, 250 \mathrm{MHz}$

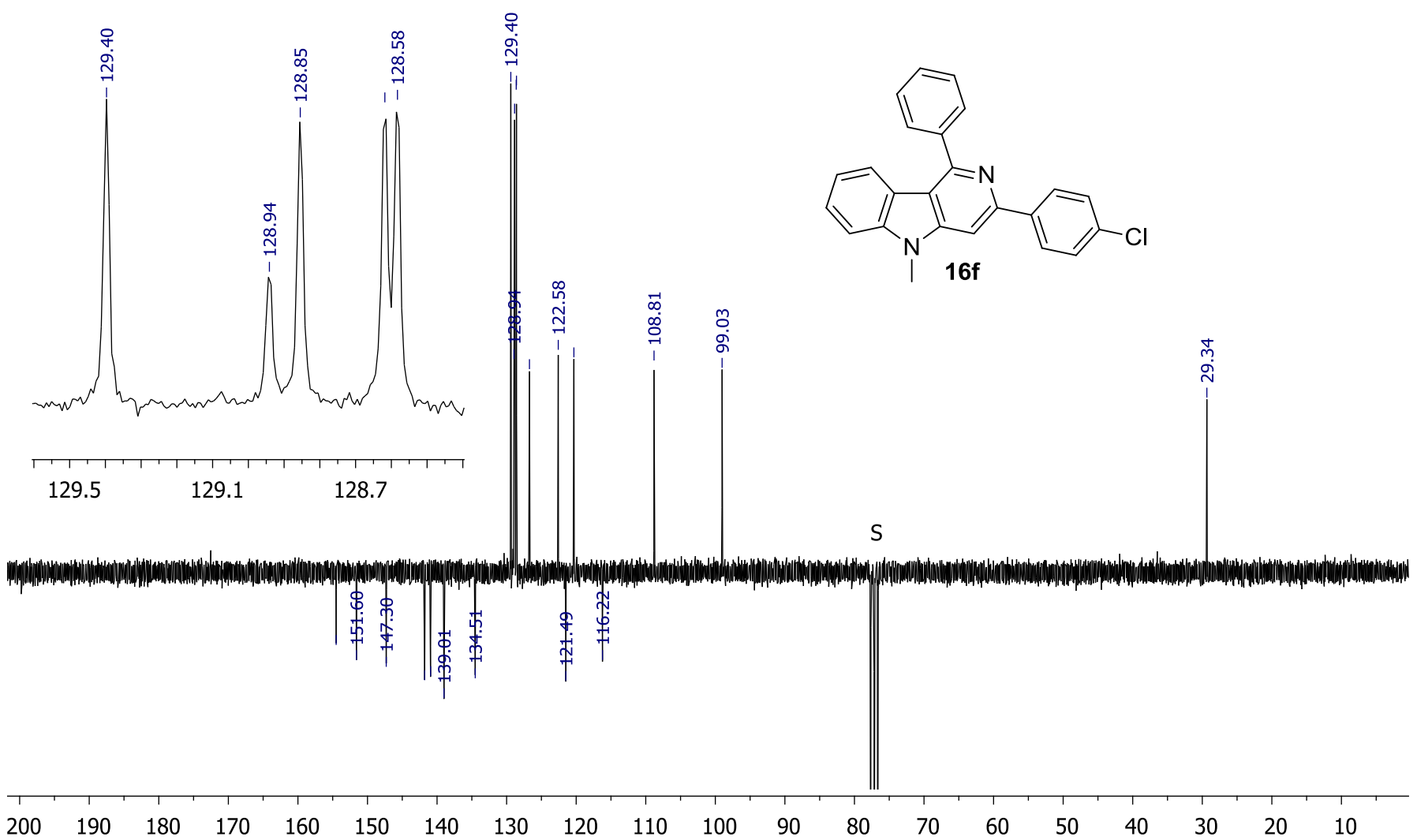

${ }^{13} \mathrm{C} \mathrm{NMR}, \mathrm{CDCl}_{3}, 62.9 \mathrm{MHz}$ 


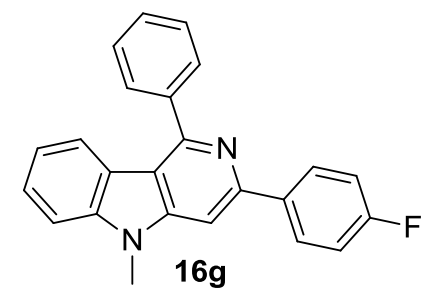

acetone

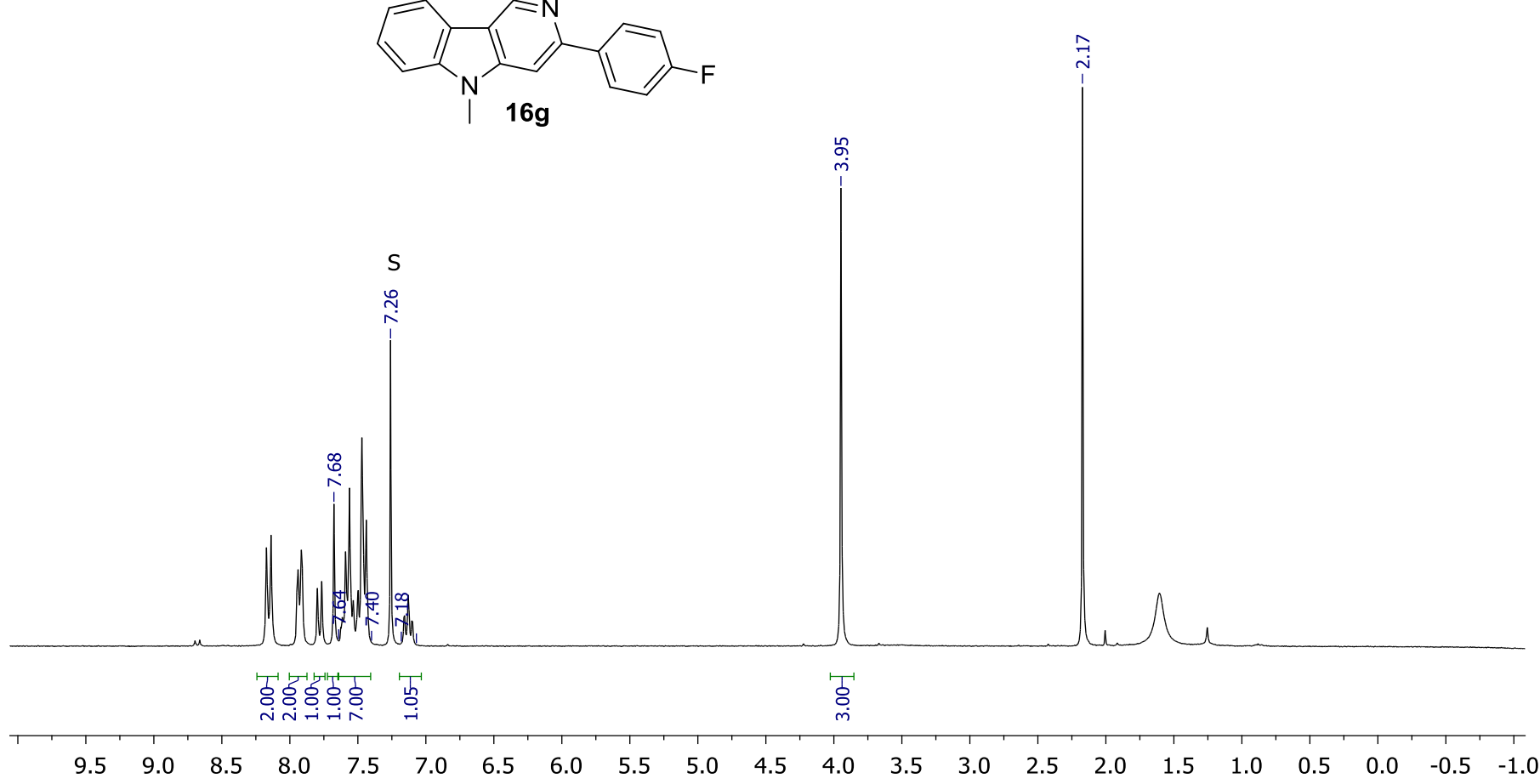

${ }^{1} \mathrm{H} \mathrm{NMR}, \mathrm{CDCl}_{3}, 250 \mathrm{MHz}$

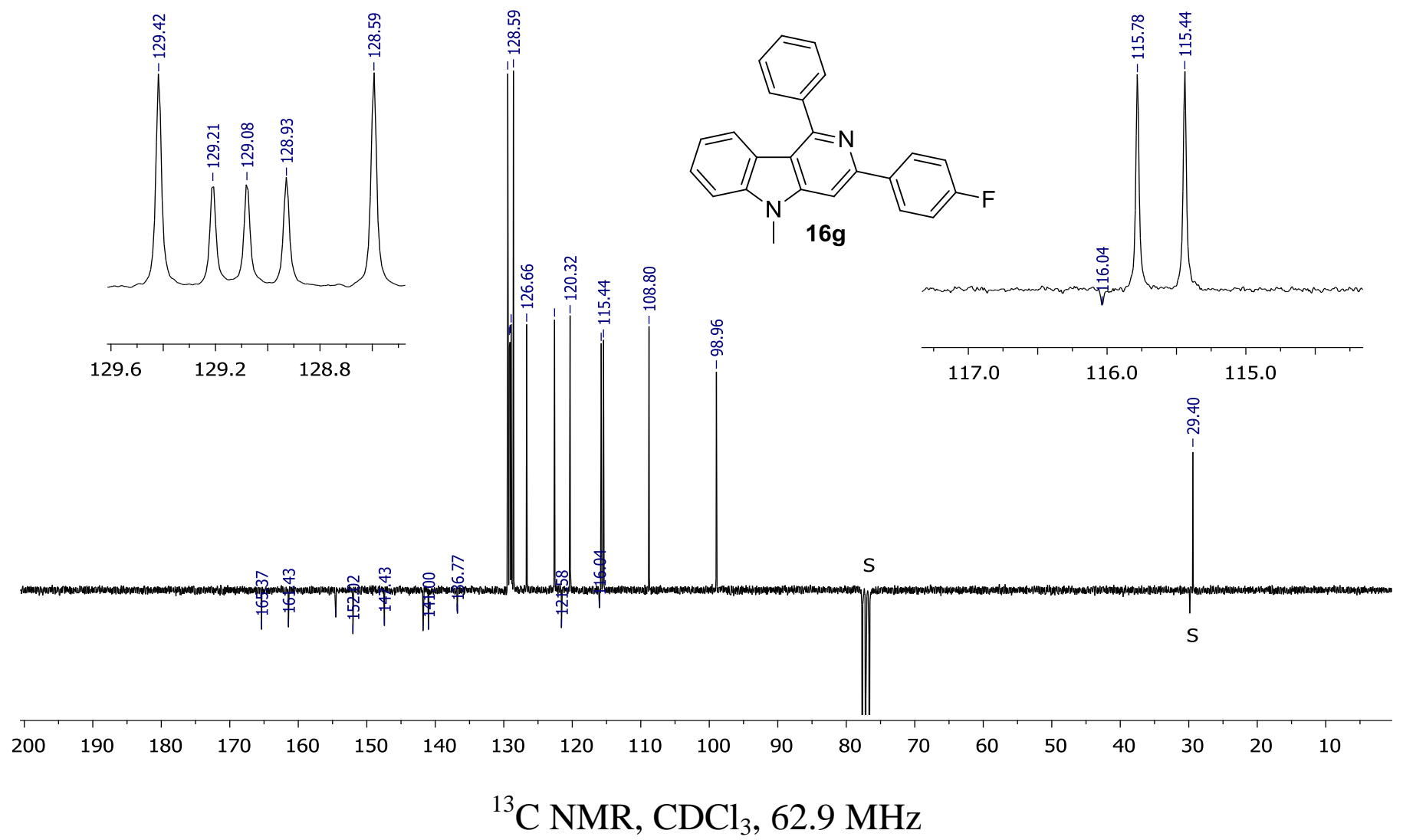




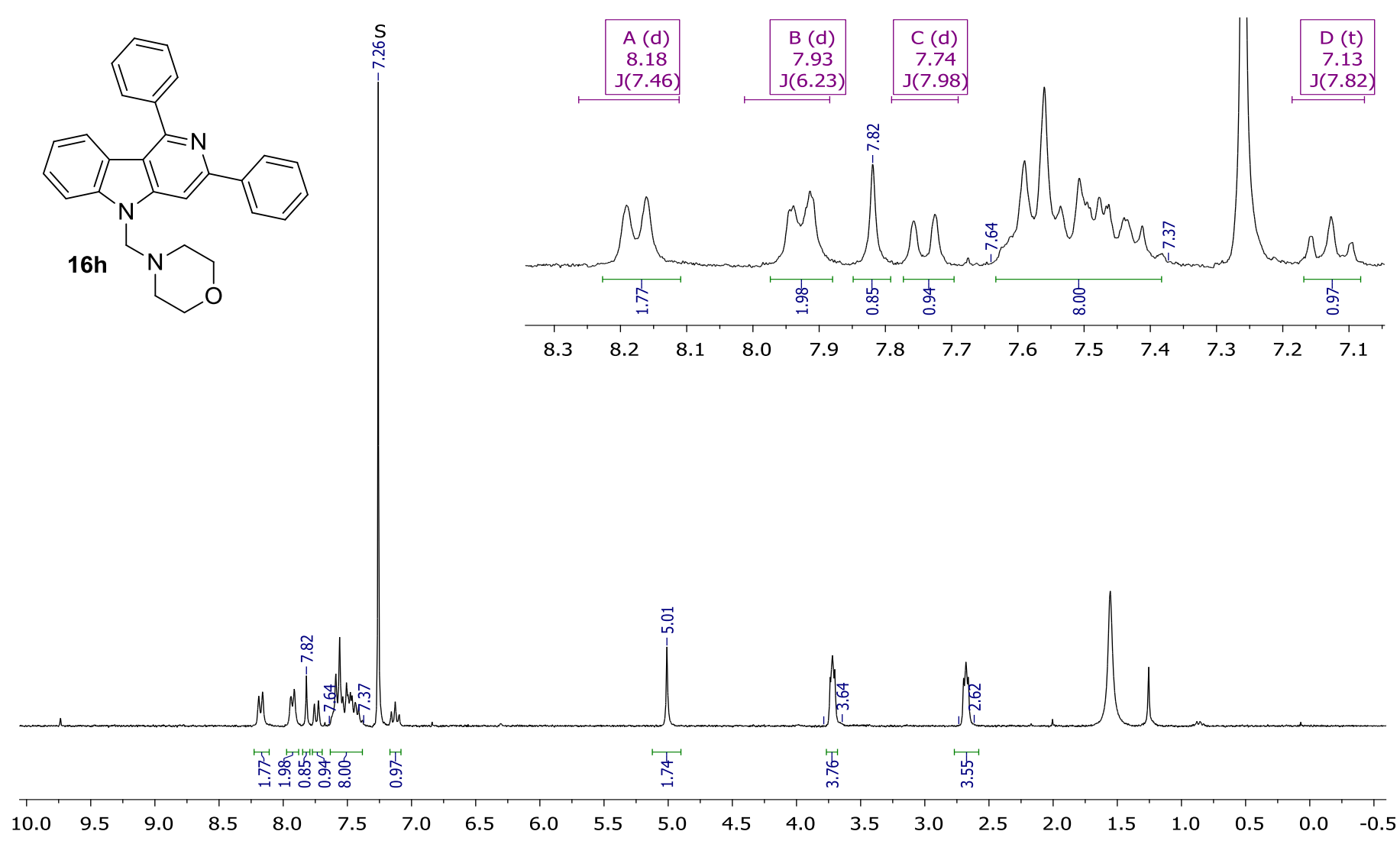

\section{${ }^{1} \mathrm{H} \mathrm{NMR}, \mathrm{CDCl}_{3}, 250 \mathrm{MHz}$}

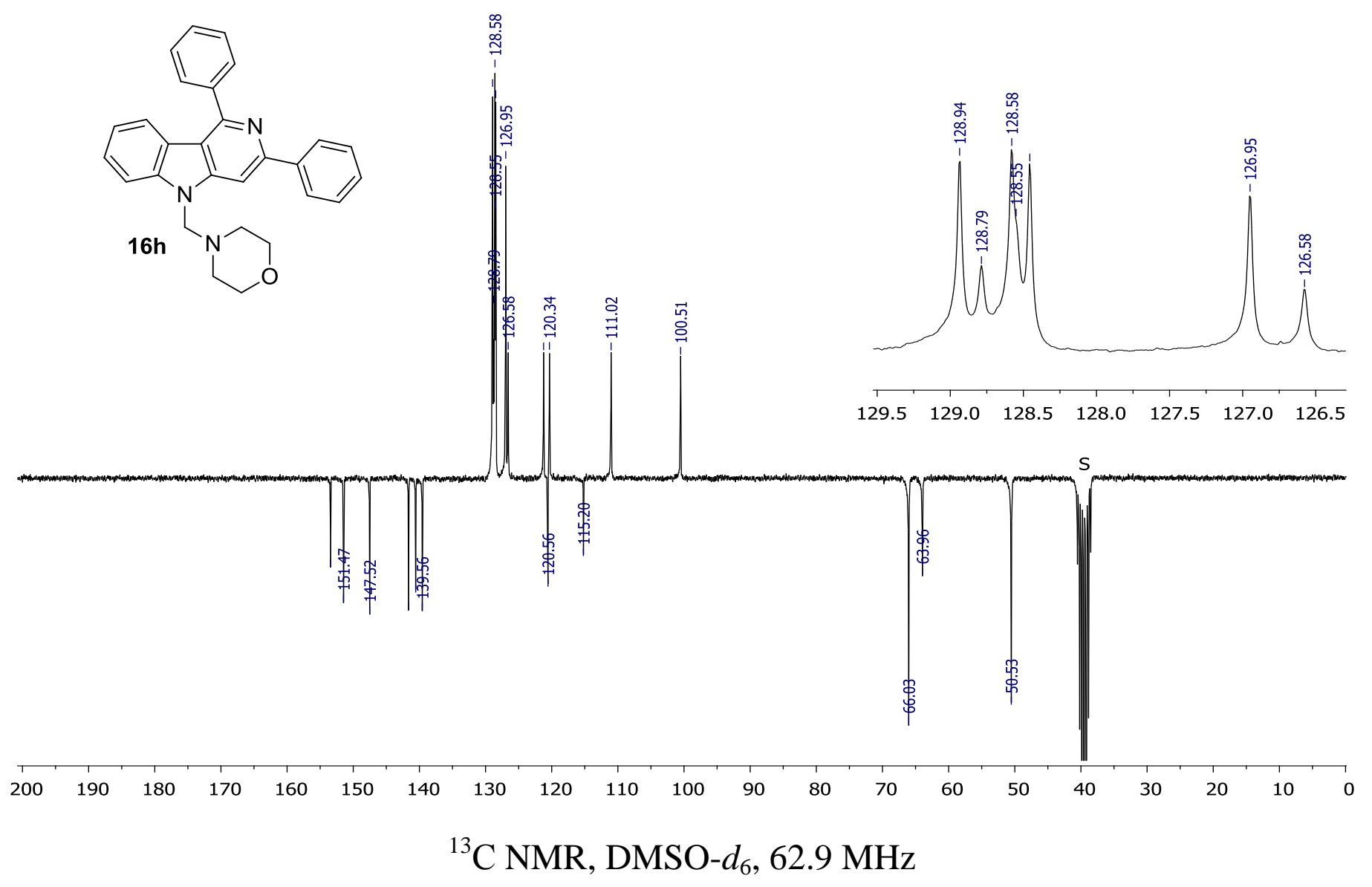




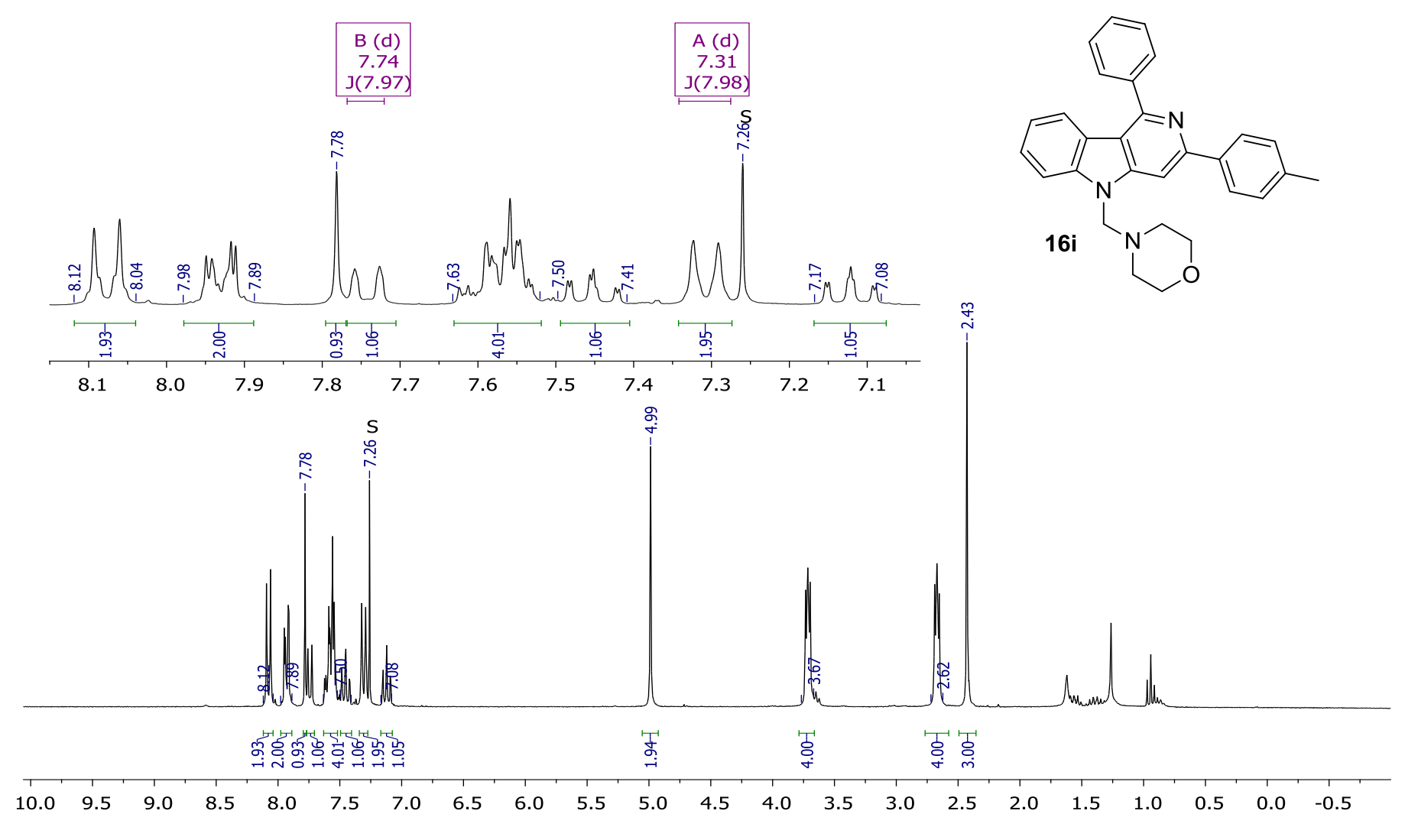

${ }^{1} \mathrm{H} \mathrm{NMR}, \mathrm{CDCl}_{3}, 250 \mathrm{MHz}$
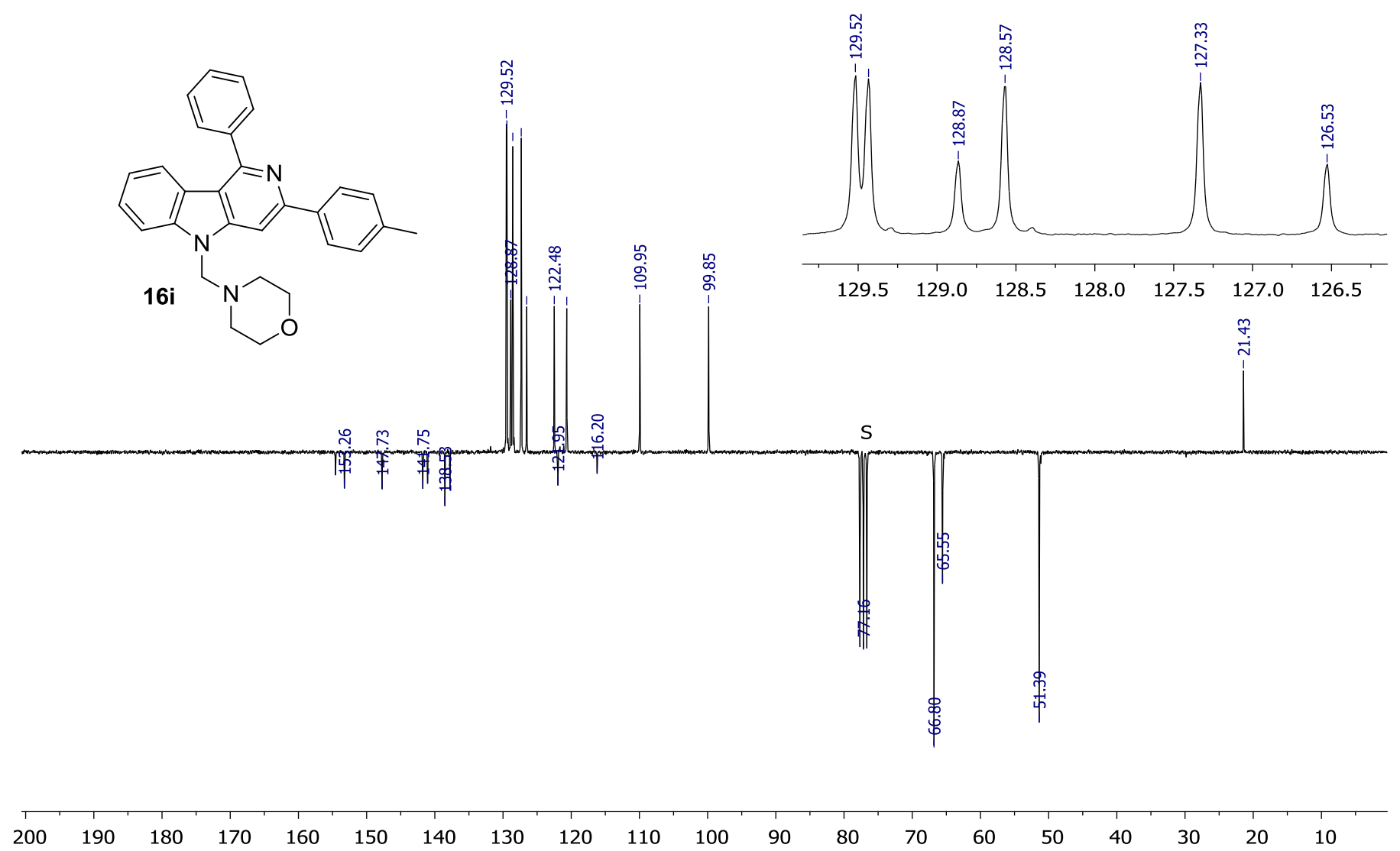

${ }^{13} \mathrm{C} \mathrm{NMR}, \mathrm{CDCl}_{3}, 62.9 \mathrm{MHz}$ 

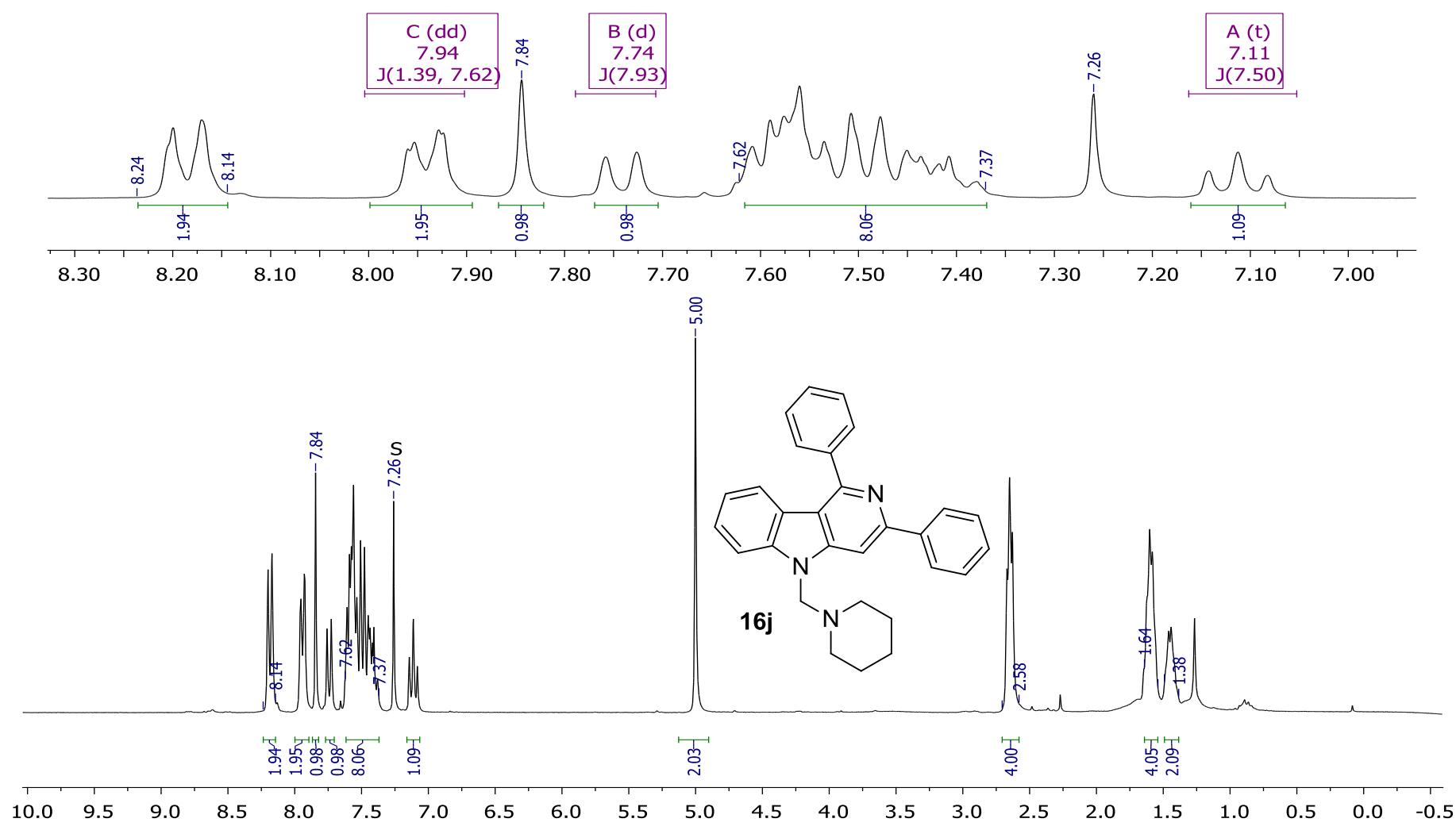

${ }^{1} \mathrm{H} \mathrm{NMR}, \mathrm{CDCl}_{3}, 250 \mathrm{MHz}$

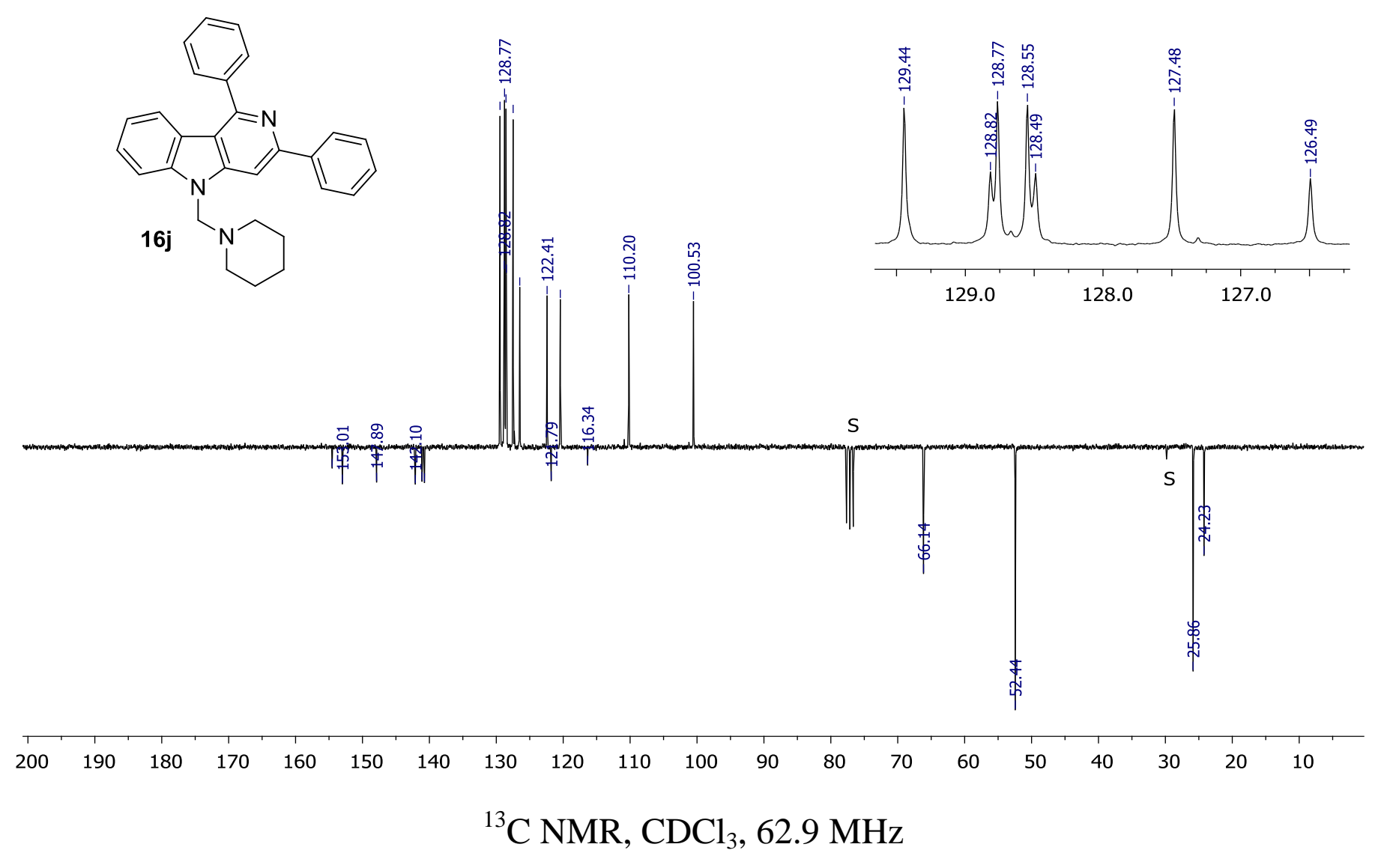



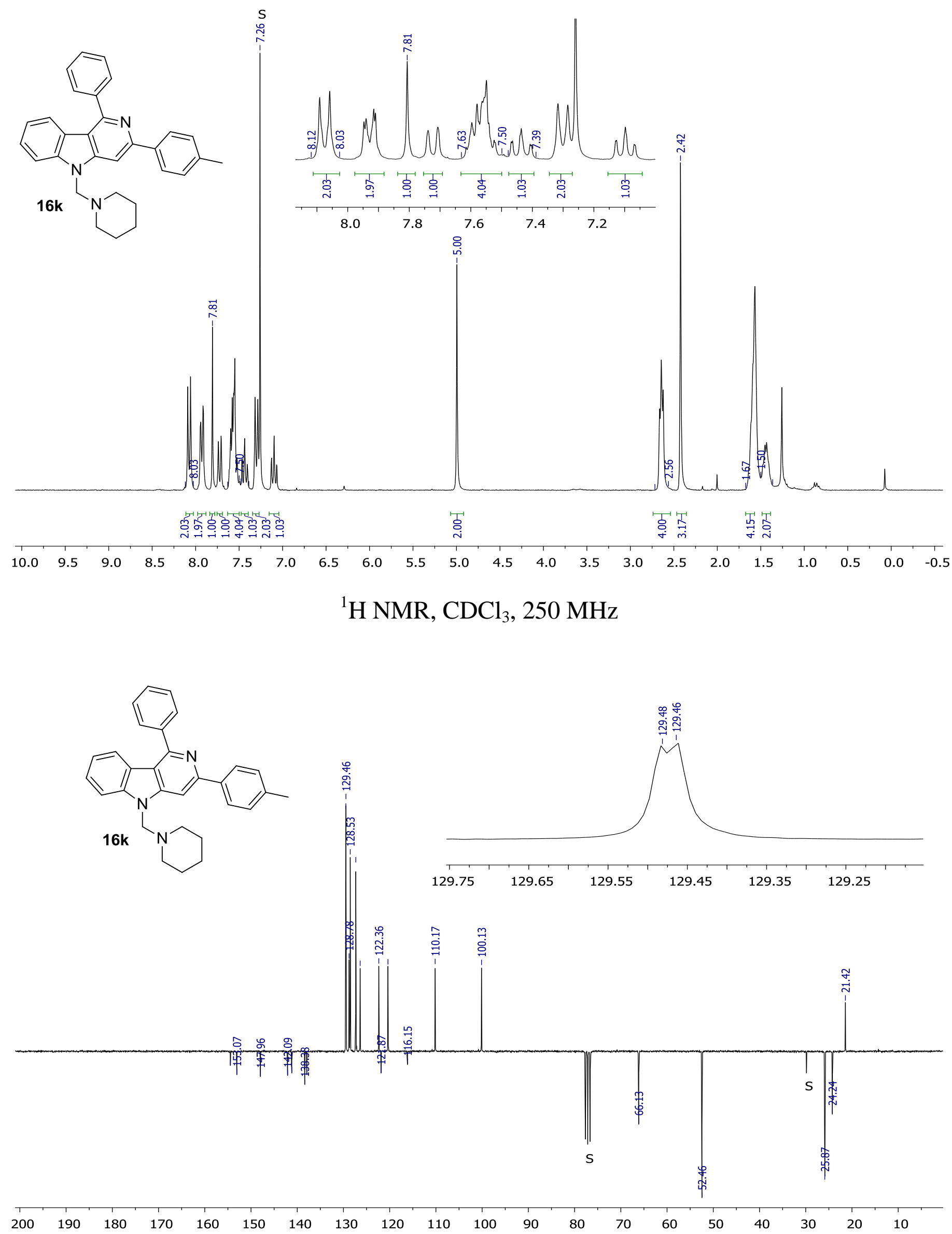

${ }^{13} \mathrm{C} \mathrm{NMR}_{\mathrm{NDCl}}, 62.9 \mathrm{MHz}$ 

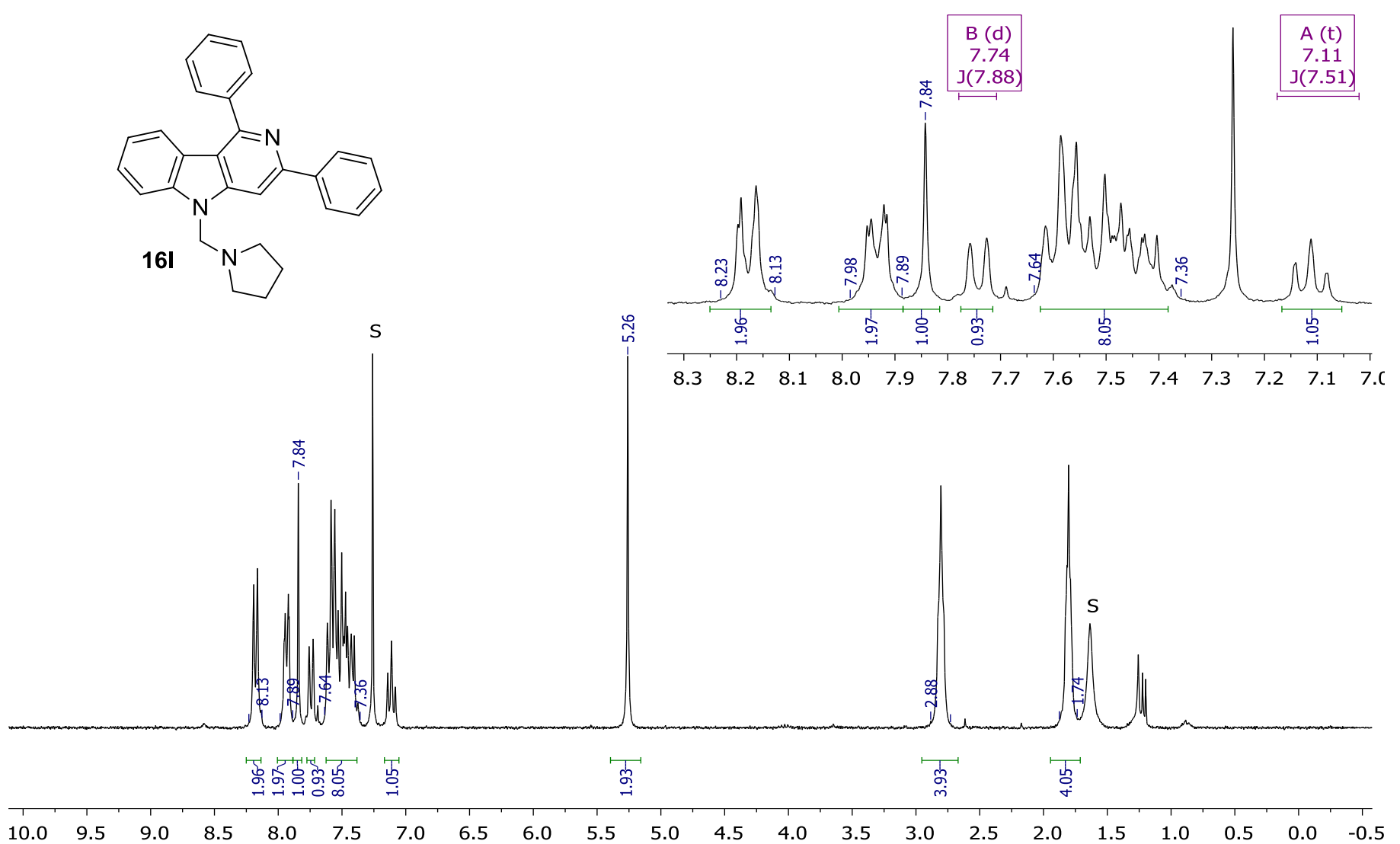

${ }^{1} \mathrm{H} \mathrm{NMR}, \mathrm{CDCl}_{3}, 250 \mathrm{MHz}$
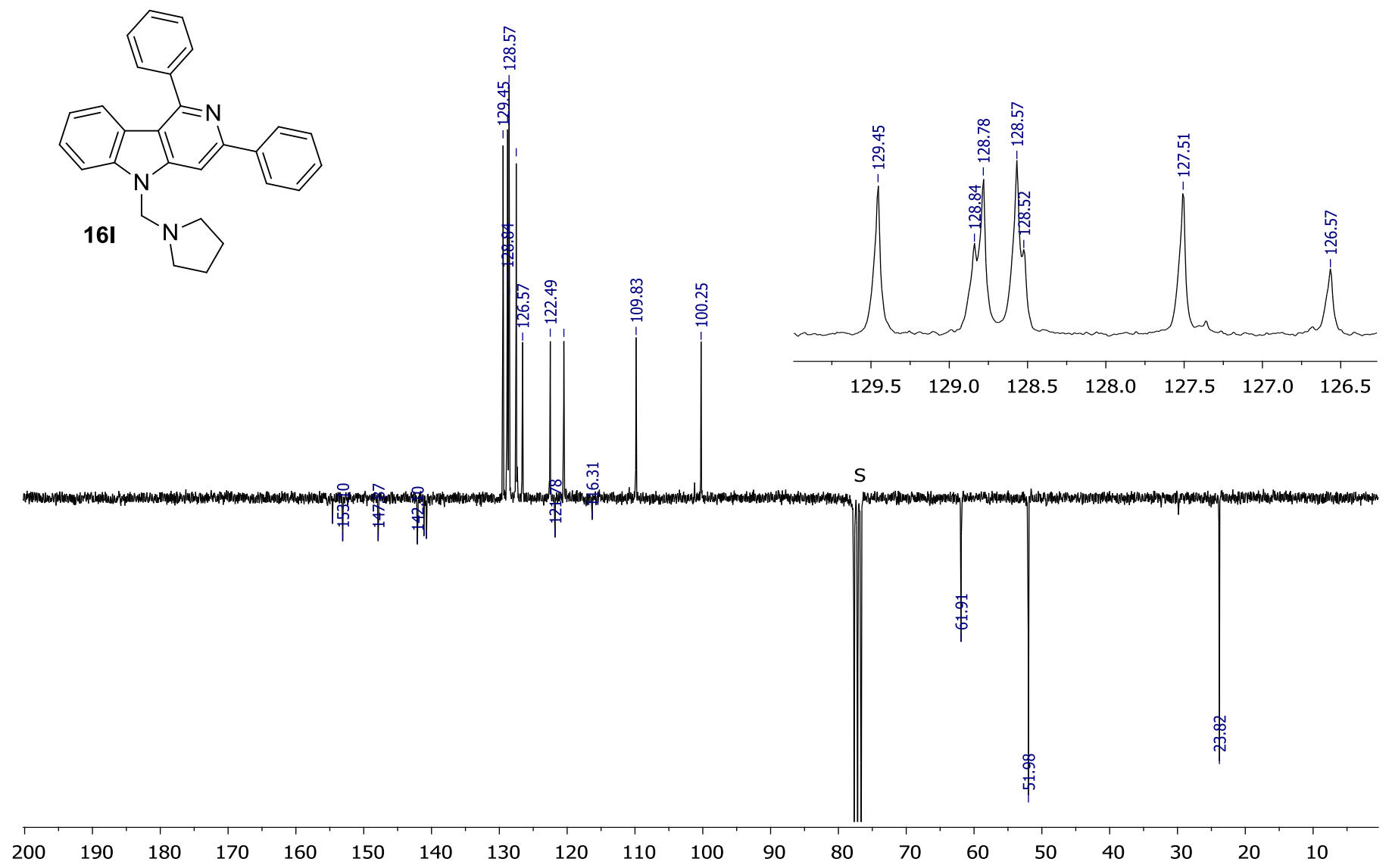

${ }^{13} \mathrm{C} \mathrm{NMR}, \mathrm{CDCl}_{3}, 62.9 \mathrm{MHz}$ 


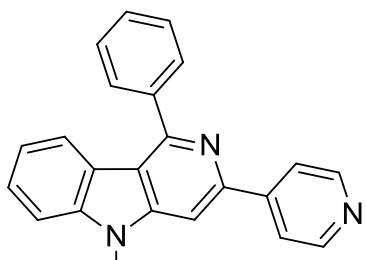

$16 \mathrm{~m}$
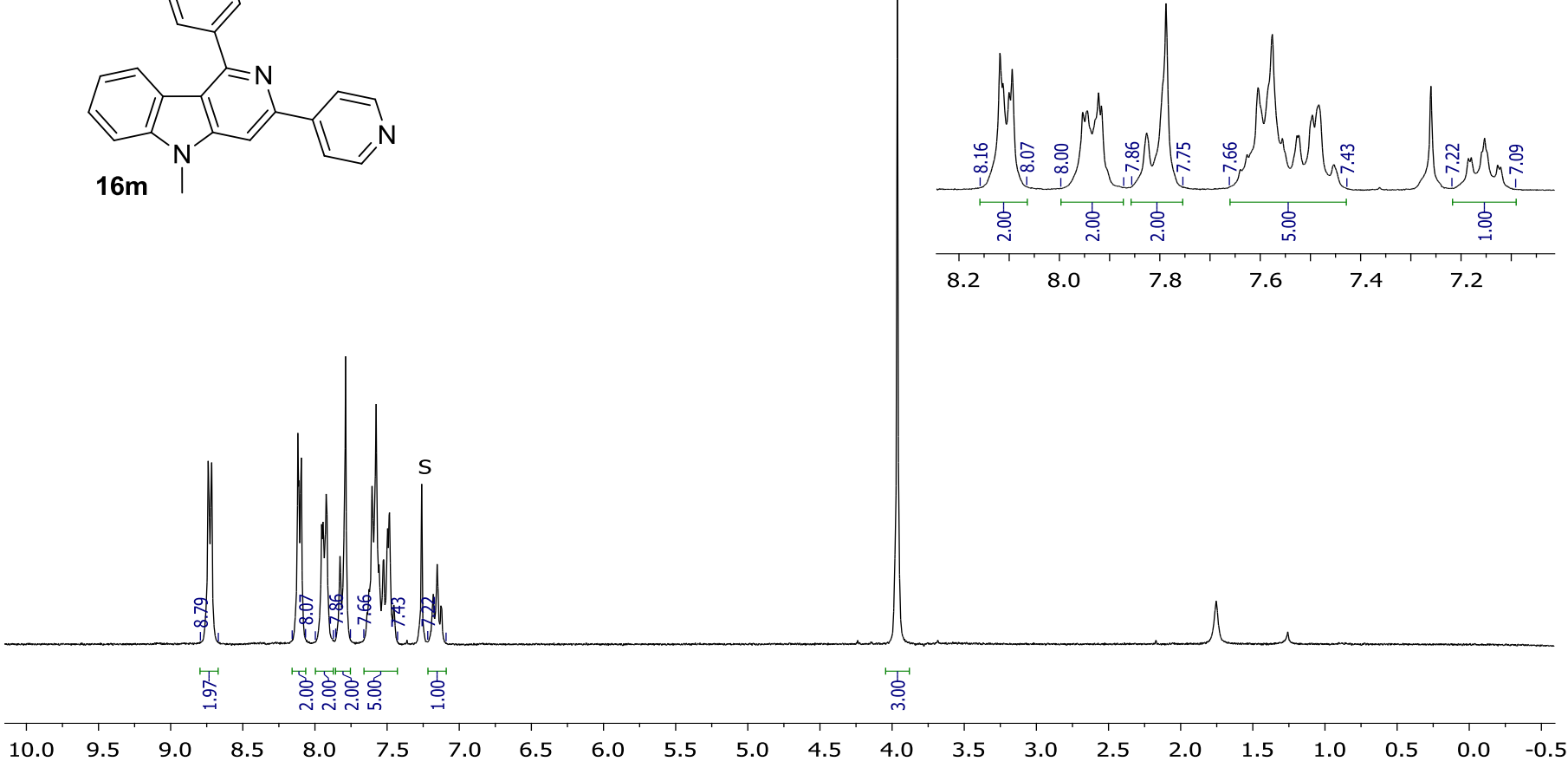

${ }^{1} \mathrm{H}$ NMR, $\mathrm{CDCl}_{3}, 250 \mathrm{MHz}$

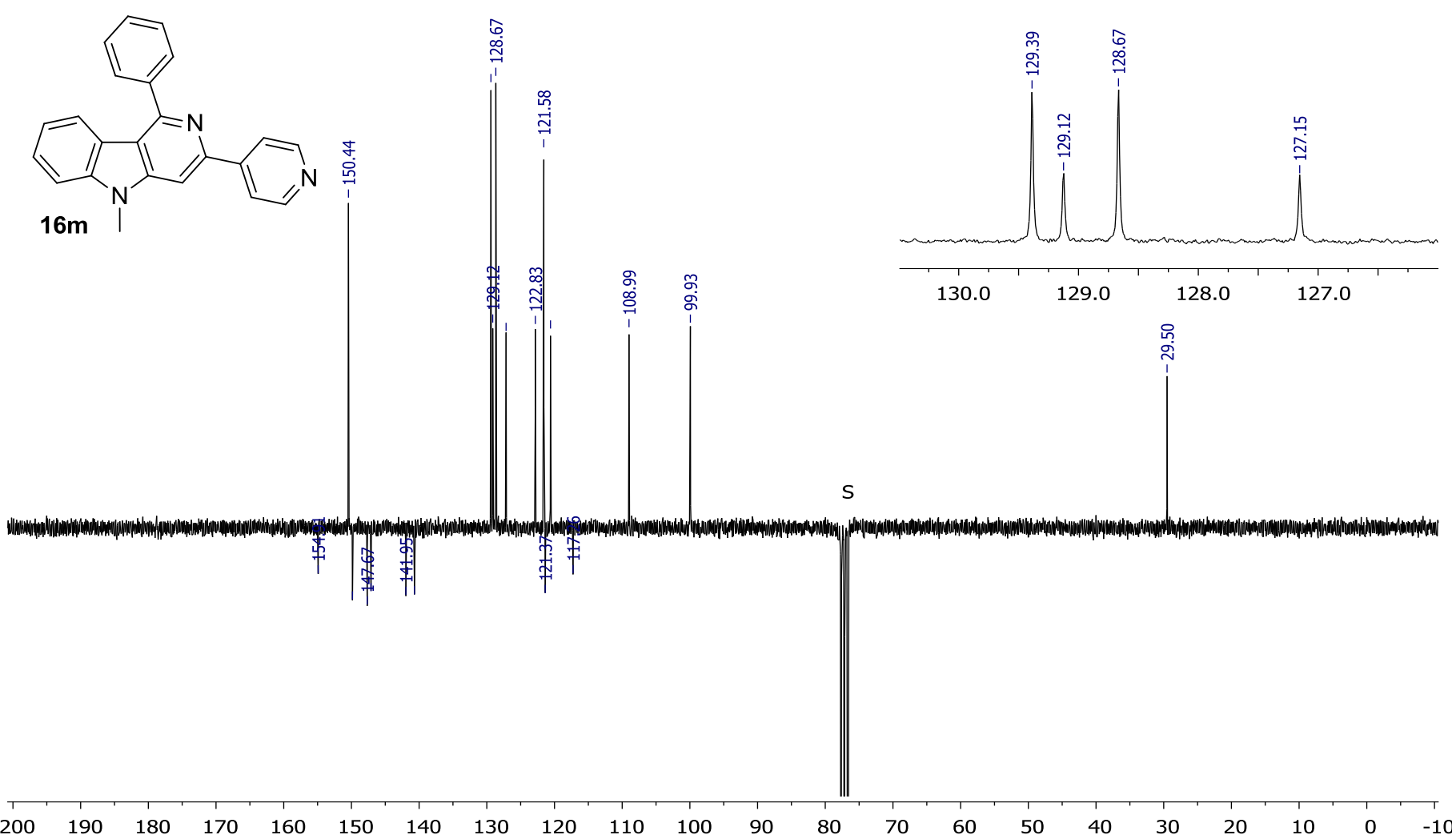

${ }^{13} \mathrm{C} \mathrm{NMR}, \mathrm{CDCl}_{3}, 62.9 \mathrm{MHz}$ 
<smiles>Cn1c2ccccc2c2c(-c3ccccc3)nc(-c3cccnc3)cc21</smiles>

$16 n$

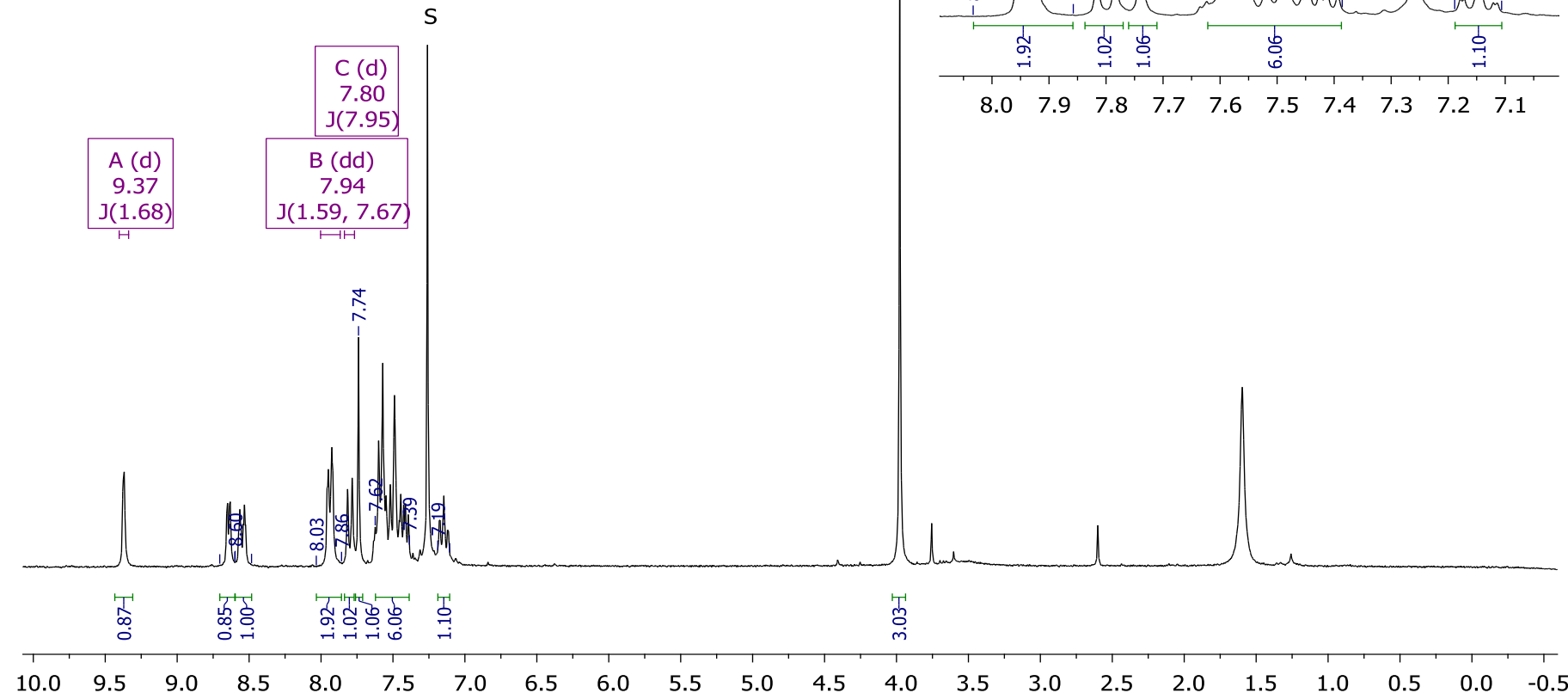

${ }^{1} \mathrm{H} \mathrm{NMR}, \mathrm{CDCl}_{3}, 250 \mathrm{MHz}$
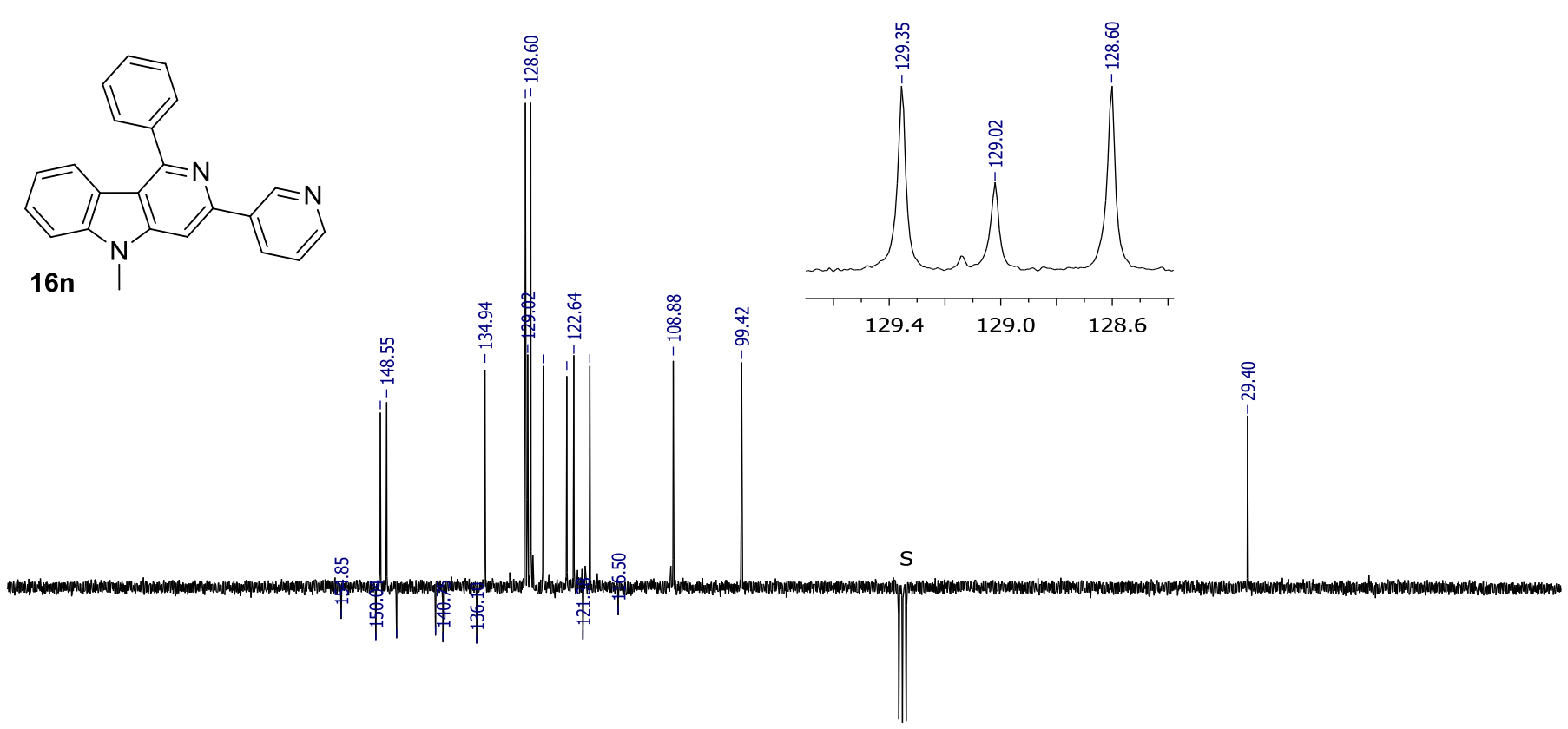

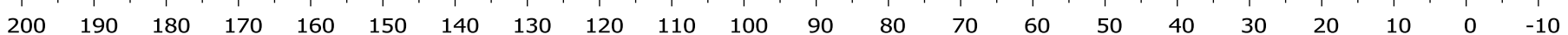

${ }^{13} \mathrm{C} \mathrm{NMR}, \mathrm{CDCl}_{3}, 62.9 \mathrm{MHz}$ 

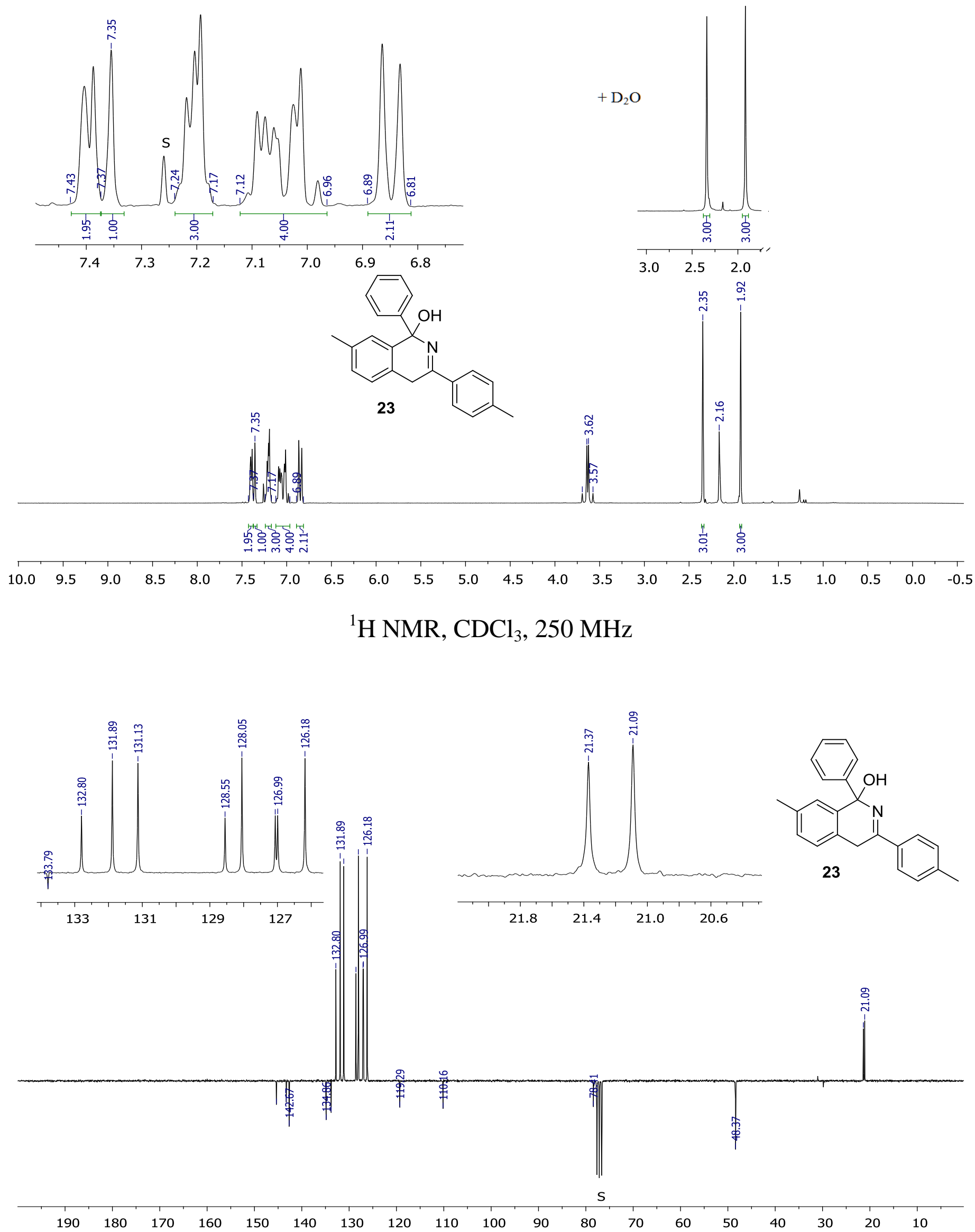

${ }^{13} \mathrm{C} \mathrm{NMR} \mathrm{CDCl}_{3}, 62.9 \mathrm{MHz}$ 


\section{References}

(1) (a) Becke, A. D. Density-functional exchange-energy approximation with correct asymptotic behavior. Phys. Rev. A. 1988, 38, 3098-3100. (b) Becke, A. D. Density-functional thermochemistry. III. The role of exact exchange. J. Chem. Phys. 1993, 98, 5648-5652. (c) Lee, Ch.; Yang, W.; Parr, R. G. Development of the Colle-Salvetti correlation-energy formula into a functional of the electron density. Phys. Rev. B. 1988, 37, 785-789.

(2) (a) Hu, J.-W.; Zhuang, Yu.; Luo, J.; Wei, X.-H.; Huang, X.-F. A theoretical study on reductive debromination of polybrominated diphenyl ethers. Int. J. Mol. Sci. 2012, 13, 93329342. (b) Li, L.; Hu, J.; Shi, X.; Ruan, W.; Luo, J.; Wei. X. Theoretical studies on structures, properties and dominant debromination pathways for selected polybrominated diphenyl ethers. Int. J. Mol. Sci. 2016, 17, 927. (c) Luo, J.; Hu, J.; Zhuang, Y.; Wei, X.; Huang, X. Electron-induced reductive debromination of 2,3,4-tribromodiphenyl ether: a computational study. J. Mol. Model. 2013, 19, 3333-3338.

(3) (a) Quantum chemical and statistical theory of solutions: a computational approach; Simkin, B. Y., Sheikhet, I. I.; Ellis Horwood: London, 1995. (b) Cances, E.; Mennucci, B.; Tomasi, J. A new integral equation formalism for the polarizable continuum model: Theoretical background and applications to isotropic and anisotropic dielectrics. J. Chem. Phys. 1997, 107, 3032-3041. (c) Cossi, M.; Barone, V.; Cammi, R.; Tomasi, J. Ab initio study of solvated molecules: a new implementation of the polarizable continuum model. $J$. Chem. Phys. Lett. 1996, 255, 327-335. (d) Barone, V.; Cossi, M.; Tomasi, J. Geometry optimization of molecular structures in solution by the polarizable continuum model. $J$. Comput. Chem. 1998, 19, 404-417.

(4) Frisch, M. J.; Trucks, G. W.; Schlegel, H. B.; Scuseria, G. E.; Robb, M. A.; Cheeseman, J. R.; Scalmani, G.; Barone, V.; Mennucci, B.; Petersson, G. A.; Nakatsuji, H.; Caricato, M.; Li, X.; Hratchian, H. P.; Izmaylov, A. F.; Bloino, J.; Zheng, G.; Sonnenberg, J. L.; Hada, M.; Ehara, M.; Toyota, K.; Fukuda, R.; Hasegawa, J.; Ishida, M.; Nakajima, T.; Honda, Y.; Kitao, O.; Nakai, H.; Vreven, T.; Montgomery, J. A., Jr.; Peralta, J. E.; Ogliaro, F.; Bearpark, M.; Heyd, J. J.; Brothers, E.; Kudin, K. N.; Staroverov, V. N.; Kobayashi, R.; Normand, J.; Raghavachari, K.; Rendell, A.; Burant, J. C.; Iyengar, S. S.; Tomasi, J.; Cossi, M.; Rega, N.; Millam, J. M.; Klene, M.; Knox, J. E.; Cross, J. B.; Bakken, V.; Adamo, C.; Jaramillo, J.; Gomperts, R.; Stratmann, R. E.; Yazyev, O.; Austin, A. J.; Cammi, R.; Pomelli, C.; Ochterski, J. W.; Martin, R. L.; Morokuma, K.; Zakrzewski, V. G.; Voth, G. A.; Salvador, P.; Dannenberg, J. J.; Dapprich, S.; Daniels, A. D.; Farkas, O.; Foresman, J. B.; Ortiz, J. V.; Cioslowski, J.; Fox, D. J. Gaussian, 09; Gaussian, Inc.: Wallingford CT, 2009.

(5) Bader, R. F. W. A quantum theory of molecular structure and its applications. Chem. Rev. 1991, 91, 893-928. 